San Jose State University

SJSU ScholarWorks

Faculty Publications

Physics and Astronomy

$5-6-2009$

\title{
Mapping the dark side with DEIMOS: globular clusters, X-ray gas, and dark matter in the NGC 1407 group
}

Aaron J. Romanowsky

San Jose State University, aaron.romanowsky@sjsu.edu

J. Strader

uCO/Lick Observatory

L. Spitler

Swinburne University

R. Johnson

University of Birmingham

J. P. Brodie

UCO/Lick Observatory

See next page for additional authors

Follow this and additional works at: https://scholarworks.sjsu.edu/physics_astron_pub

Part of the Astrophysics and Astronomy Commons

\section{Recommended Citation}

Aaron J. Romanowsky, J. Strader, L. Spitler, R. Johnson, J. P. Brodie, D. A. Forbes, and T. Ponman.

"Mapping the dark side with DEIMOS: globular clusters, X-ray gas, and dark matter in the NGC 1407 group" Astronomical Journal (2009): 4956-4987. https://doi.org/10.1088/0004-6256/137/6/4956

This Article is brought to you for free and open access by the Physics and Astronomy at SJSU ScholarWorks. It has been accepted for inclusion in Faculty Publications by an authorized administrator of SJSU ScholarWorks. For more information, please contact scholarworks@sjsu.edu. 


\section{Authors}

Aaron J. Romanowsky, J. Strader, L. Spitler, R. Johnson, J. P. Brodie, D. A. Forbes, and T. Ponman 


\title{
MAPPING THE DARK SIDE WITH DEIMOS: GLOBULAR CLUSTERS, X-RAY GAS, AND DARK MATTER IN THE NGC 1407 GROUP
}

\author{
Aaron J. Romanowsky ${ }^{1,2}$, Jay Strader ${ }^{1,3,6}$, Lee R. Spitler ${ }^{4}$, Ria Johnson ${ }^{5}$, Jean P. Brodie ${ }^{1}$, Duncan A. Forbes ${ }^{4}$, \\ AND TREVOR PONMAN 5 \\ ${ }^{1}$ UCO/Lick Observatory, University of California, Santa Cruz, CA 95064, USA; romanow@ucolick.org \\ ${ }^{2}$ Departamento de Física, Universidad de Concepción, Casilla 160-C, Concepción, Chile \\ ${ }^{3}$ Harvard-Smithsonian Center for Astrophysics, 60 Garden Street, Cambridge, MA 02138, USA \\ ${ }^{4}$ Centre for Astrophysics \& Supercomputing, Swinburne University, Hawthorn, VIC 3122, Australia \\ ${ }^{5}$ School of Physics and Astronomy, University of Birmingham, Edgbaston, Birmingham B15 2TT, UK \\ Received 2008 June 27; accepted 2009 March 16; published 2009 May 6
}

\begin{abstract}
NGC 1407 is the central elliptical in a nearby evolved group of galaxies apparently destined to become a galaxy cluster core. We use the kinematics of globular clusters (GCs) to probe the dynamics and mass profile of the group's center, out to a radius of $60 \mathrm{kpc}(\sim 10$ galaxy effective radii) - the most extended data set to date around an early-type galaxy. This sample consists of $172 \mathrm{GC}$ line-of-sight velocities, most of them newly obtained using Keck/DEIMOS, with a few additional objects identified as dwarf-globular transition objects or as intragroup GCs. We find weak rotation for the outer parts of the GC system $(v / \sigma \sim 0.2)$, with a rotational misalignment between the metal-poor and metal-rich GCs. The velocity dispersion profile declines rapidly to a radius of $\sim 20 \mathrm{kpc}$, and then becomes flat or rising to $\sim 60 \mathrm{kpc}$. There is evidence that the GC orbits have a tangential bias that is strongest for the metal-poor GCs-in possible contradiction to theoretical expectations. We construct cosmologically motivated galaxy+dark halo dynamical models and infer a total mass within $60 \mathrm{kpc}$ of $\sim 3 \times 10^{12} M_{\odot}$, which extrapolates to a virial mass of $\sim 6 \times 10^{13} M_{\odot}$ for a typical lambda cold dark matter $(\Lambda \mathrm{CDM})$ halo-in agreement with results from kinematics of the group galaxies. We present an independent Chandra-based analysis, whose relatively high mass at $\sim 20 \mathrm{kpc}$ disagrees strongly with the GC-based result unless the GCs are assumed to have a peculiar orbit distribution, and we therefore discuss more generally some comparisons between X-ray and optical results. The group's $B$-band mass-to-light ratio of $\sim 800$ (uncertain by a factor of $\sim 2$ ) in Solar units is extreme even for a rich galaxy cluster, much less a poor group-placing it among the most dark matter (DM) dominated systems in the universe, and also suggesting a massive reservoir of baryons lurking in an unseen phase, in addition to the nonbaryonic DM. We compare the kinematical and mass properties of the NGC 1407 group to other nearby groups and clusters, and discuss some implications of this system for structure formation.
\end{abstract}

Key words: dark matter - galaxies: clusters: general - galaxies: elliptical and lenticular, cD - galaxies: kinematics and dynamics - globular clusters: general - X-rays: galaxies

Online-only material: color figures, machine-readable and VO tables

\section{INTRODUCTION}

In our current understanding of the universe, stellar systems larger than a globular cluster (GC; baryon mass $\sim 10^{6} M_{\odot}$ ) are generally embedded in massive halos of dark matter (DM). Their systemic properties versus increasing DM halo mass show that there must be shifts between the various regimes of physical processes shaping the baryonic properties (e.g., Rees \& Ostriker 1977; Benson et al. 2003; Maller \& Bullock 2004; Dekel \& Birnboim 2006; Kang et al. 2007; Nipoti \& Binney 2007; Gilbank \& Balogh 2008). In particular, somewhere between the scales of individual galaxies and clusters of galaxies, the mass of a halo's primary galaxy stops scaling with the halo mass, and reaches a universal upper limit (see, e.g., Rocca-Volmerange et al. 2004; Cooray \& Milosavljević 2005; Bernardi et al. 2006; Kirby et al. 2008; Hansen et al. 2009), while the bulk of the baryons become spread out as a hot intergalactic medium (IGM). The high-mass systems behave less like galaxies with their own enveloping halos and unique histories, and more like extended halos shaping the destinies of their captive galaxian members (e.g., Forbes et al. 2006b).

\footnotetext{
6 Hubble Fellow.
}

In collapsed galaxy clusters, the dominant processes affecting the larger galaxies involve cooling, heating, and stripping by the IGM, while some level of harassment and stripping also occurs by galaxy flybys within the cluster. However, it is thought that processes such as gas strangulation and major galaxy mergers occurred earlier, within the groups that went on to conglomerate into the cluster (e.g., Barnes 1985; Zabludoff \& Mulchaey 1998; Kodama \& Smail 2001; Carlberg et al. 2001; Fujita 2004; Brough et al. 2006a, hereafter B+06a; Moran et al. 2007; Kawata \& Mulchaey 2008; Wilman et al. 2008; Kautsch et al. 2008; Patel et al. 2009). In this picture, the characteristic properties of cluster galaxies (dominated by early-types) would primarily arise by "preprocessing" in groups. Thus, by studying galaxy groups, one can learn not only about the ecosystems of most of the galaxies in the universe (Eke et al. 2004a), but also about the prehistory of the rarer but interesting galaxy clusters.

The nearby group surrounding the elliptical galaxy NGC 1407, sometimes called the "Eridanus A" group (Willmer et al. 1989; Ferguson \& Sandage 1990; Brough et al. 2006b, hereafter $\mathrm{B}+06 \mathrm{~b})$, is a potentially informative transitional system. It has the X-ray and optical luminosities of a group (with 1-3 galaxies of at least $L^{*}$ luminosity, and highly dominated by 
early-types), but possibly the mass of a cluster. An early study of its constituent galaxy redshifts suggested a group mass-tolight ratio of $\Upsilon \sim 2500 \Upsilon_{\odot}$ ( $B$ band; Gould 1993), and while subsequent studies have brought this estimate down to $\sim 300$ 1100 (partially through longer distance estimates; Quintana et al. 1994; Tully 2005; B+06b; Trentham et al. 2006; Firth et al. 2006; Zhang et al. 2007, hereafter $Z+07)$, these are still high values more reminiscent of clusters than of groups.

The NGC 1407 group may provide insight into the origins of the properties of galaxy cluster cores and of brightest cluster galaxies. The group is part of a larger structure or "supergroup" that should eventually collapse to form a cluster with NGC 1407 - and possibly its associated group — at its core $(\mathrm{B}+06 \mathrm{~b}$; Trentham et al. 2006). Major mergers are prone to scramble the memories of their progenitors' prior properties, but the future assembly of the NGC 1407 cluster appears to involve low mass ratios of $\sim 1: 5-1: 10$. Thus, it is likely that the NGC 1407 group will remain relatively unscathed at the core of the resulting cluster, and we can make a fair comparison of its current dynamical properties with those of existing cluster cores (such as M87 in Virgo and NGC 1399 in Fornax) to see if these systems are part of the same evolutionary sequence.

NGC 1407, like many bright elliptical galaxies, is endowed with a swarm of GCs visible as bright sources extending far out into its halo, beyond the region of readily observable galaxy light. GCs are invaluable as tracers of major episodes of star formation in galaxies (Whitmore \& Schweizer 1995; Larsen \& Richtler 2000; Brodie \& Strader 2006). In NGC 1407, Cenarro et al. (2007, hereafter C+07) have used them to study the galaxy's early metal enrichment history, and to search for signs of recent gas-rich mergers. Here, our focus is on using GCs as bountiful kinematical tracers, useful for probing both the dynamical properties of their host galaxy's outer parts, and the mass distribution of the surrounding group.

The use of GCs as mass tracers is well established: see Brodie \& Strader (2006) and Romanowsky (2009) for reviews, and Section 5.2 of this paper for specific examples. GCs are so far less widely exploited as orbit tracers-using the internal dynamics of a globular cluster system (GCS) to infer its formation history and its connections with other galaxy subcomponents. Studies of GCS rotation have implicitly treated the GCs as proxies for the halo field stars or for the DM particles (e.g., Kissler-Patig \& Gebhardt 1998), but the connections between these different entities have not yet been established. Bekki et al. (2005, hereafter B+05) presented pioneering work in this area, deriving predictions for GCS, stellar, and DM kinematics from galaxy merger simulations. We will make some comparisons of these predictions to the data.

The $10 \mathrm{~m}$ Keck-I telescope with the LRIS multislit spectrograph (Oke et al. 1995) has produced a legacy of the first large samples of extragalactic GC kinematics outside of the Local Group (Cohen \& Ryzhov 1997; Cohen 2000; Côté et al. 2003, hereafter $\mathrm{C}+03)$. Now, we present the first use of DEIMOS on Keck-II (Faber et al. 2003) to study extragalactic GCS kinematics, representing a significant advance in this field. Its $80 \operatorname{arcmin}^{2}$ field of view is one of the most expansive available on a large telescope, and its stability, red sensitivity, and medium resolution make it highly efficient for acquiring accurate velocities for GCs (which are intrinsically red objects) even in gray skies, using the $\mathrm{Ca}$ II absorption line triplet (CaT). Our results here inaugurate the SAGES Legacy Unifying Globulars and Galaxies Survey of the detailed photometric and spectroscopic properties of $\sim 25$ representative galaxies and their GCSs.
NGC 1407 is a moderately rotating E0 galaxy, neither boxy nor disky, with a "core-like" central luminosity profile, a weak central active galactic nucleus (AGN), and absolute magnitudes of $M_{B}=-21.0$ and $M_{K}=-24.8(\mathrm{~B}+06 \mathrm{~b})$. Its GCS displays a typical color bimodality of "blue" and "red" GCs (Perrett et al. 1997; Forbes et al. 2006a; Harris et al. 2006, hereafter H+06a; Harris 2009). Its overall specific frequency of GCs per unit luminosity was previously found to be a modest $S_{N} \sim 4$, but a new deep wide-field study has produced a richer estimate of $S_{N} \sim 6$ (L. Spitler et al. 2010, in preparation, hereafter $S+10$ ). NGC 1407 hosts bright, extended X-ray emission that extends as far out as $80 \mathrm{kpc}$ into the surrounding group (B+06b; Z+07), although there is nothing about its X-ray properties that marks it as a particularly unusual galaxy (Osmond \& Ponman 2004, hereafter OP04). We adopt a galaxy stellar effective radius of $R_{\text {eff }}=57^{\prime \prime}=5.8 \mathrm{kpc}$ (see Section 4.1.1), with a distance to the galaxy and group of $20.9 \mathrm{Mpc}$ (Forbes et al. 2006a) — discussing later the effects of the distance uncertainty.

We present the spectroscopic GC observations in Section 2, and convert these into kinematical parameters in Section 3. In Section 4, we analyze the group mass profile using GC dynamics and X-ray constraints, and review independent mass constraints more generally. We place our findings for NGC 1407 into the wider context of galaxy groups in Section 5, and Section 6 summarizes the results.

\section{OBSERVATIONS AND DATA REDUCTION}

Here, we present the spectroscopic observations used in this paper, including new data from Keck/DEIMOS, and published data from Keck/LRIS. The DEIMOS observations are outlined in Section 2.1, and the data reduction in Section 2.2. The combined DEIMOS+LRIS sample is described in Section 2.3. We also make use of integrated-light stellar kinematics data for NGC 1407 from Spolaor et al. (2008a, hereafter S+08) and photometric data of the galaxy and its GCS from $\mathrm{S}+08$ (Hubble Space Telescope (HST)/ACS BI imaging) and from S+10 (Subaru/Suprime-Cam $g^{\prime} r^{\prime} i^{\prime}$ imaging; Miyazaki et al. 2002).

\subsection{Keck/DEIMOS Observations}

The DEIMOS observations were carried out as part of Keck program 2006B_U024D (PI: J. P. Brodie). Candidate GCs were selected from Subaru/Suprime-Cam images of NGC 1407 taken in $g^{\prime} r^{\prime} i^{\prime}$ bands in very good seeing conditions $\left(0^{\prime \prime} .5-0.6\right)$. The NGC 1407 GC catalog is detailed in S+10 and was supplemented with the NGC 1407 HST/ACS GC list of Forbes et al. (2006a). An astrometric solution was derived for the Subaru $g^{\prime}$-band image using unsaturated USNO-B stars in the field (Monet et al. 2003). Objects detected on the Subaru imaging were tagged as GC candidates if they met color limits of $0.4<g^{\prime}-i^{\prime} \leqslant 1.4$ and $0.3<g^{\prime}-r^{\prime} \leqslant 0.9$, and if the color values were jointly consistent with the clear linear sequence of GCs in $g^{\prime}-i^{\prime}$ versus $g^{\prime}-r^{\prime}$ color space.

For the multislit mask design, GC candidates were ranked according to their expected signal-to-noise ratio $(\mathrm{S} / \mathrm{N})$. No cuts were made on GC color beyond the initial selection of candidates. DEIMOS alignment and guide stars were also taken from the USNO-B catalog, with a preference for stars in the recommended magnitude range of $15<R<20$. To ensure a good alignment, at least two stars were placed at each end of a given DEIMOS mask. Science target slits were aligned close to 


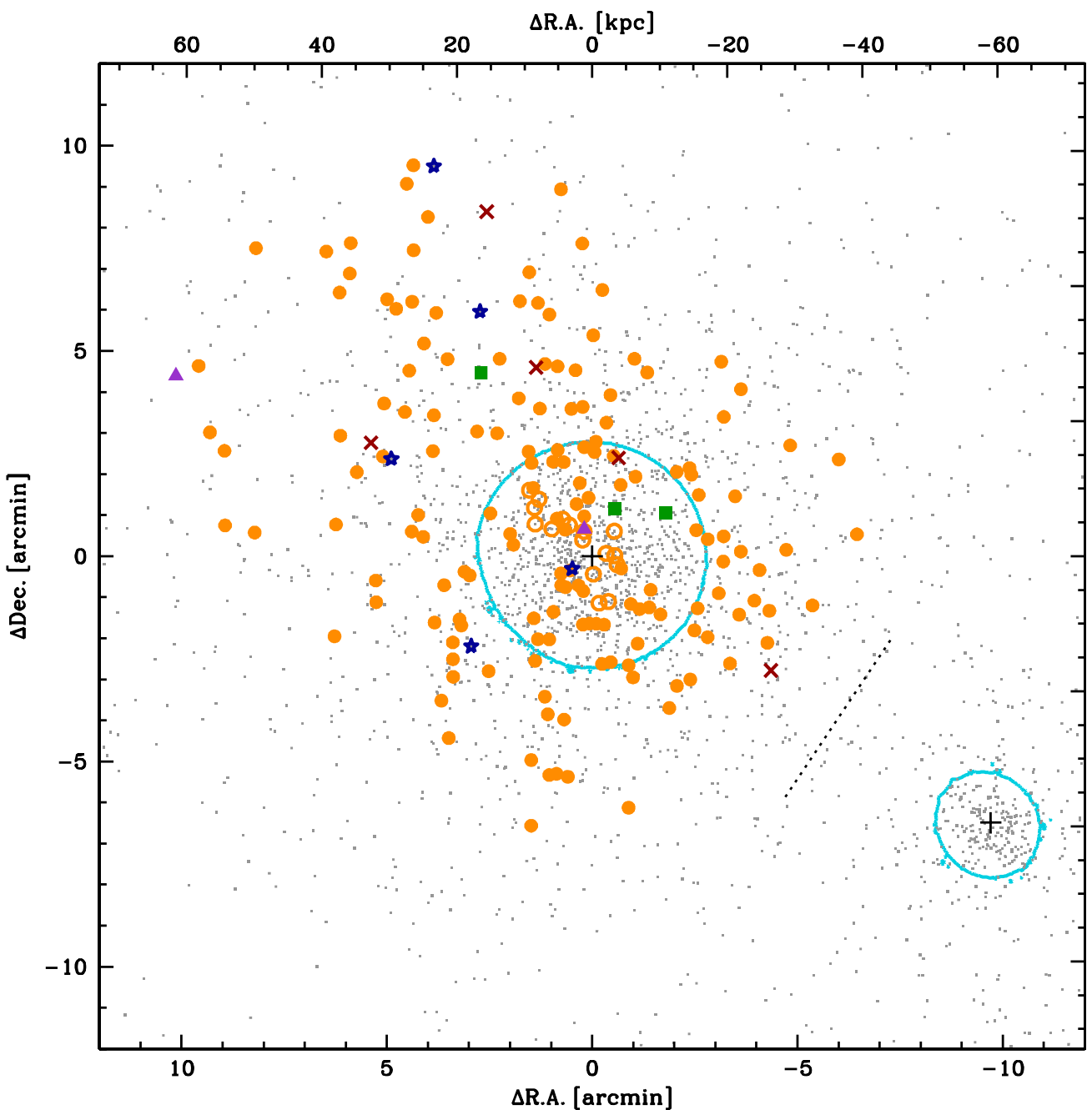

Figure 1. Overview of spectroscopic observations around NGC 1407. The filled symbols show results from DEIMOS, and open symbols from LRIS. The orange circles show positions of kinematically confirmed GCs, and the green squares show dwarf-globular transition objects. The purple triangles show borderline velocity results, red $\times$ symbols mark background galaxies, and Galactic stars are shown by blue starlike symbols. The small gray points show the underlying distribution of GC candidates from Suprime-Cam, down to the same limiting magnitude as the spectroscopically confirmed GCs; the irregular distribution of spectroscopic GCs is caused by mask and slit placement and geometry, and by poor-quality regions in the imaging. The central round contour shows the $\mu_{I}=23.2$ mag arcsec ${ }^{-2}$ stellar isophote of NGC 1407 , corresponding to $\sim 3 R_{\text {eff }}(2 ' 85)$. The lower right contour shows a similar isophote for NGC 1400 . The dotted line shows the approximate boundary of predominance between the GCSs of the two galaxies.

(A color version of this figure is available in the online journal.)

the parallactic angle, and had lengths of at least $5^{\prime \prime}$ each. Three masks provided coverage of the NGC 1407 GC system out to large radii, and were positioned to avoid potential contamination from the nearby galaxy NGC 1400 to the southwest. The GCS is fairly well sampled out to $\sim 5^{\prime}$, with an extension of $\sim 10^{\prime}$ to the northeast (see Figure 1).

Spectroscopic observations were made with Keck-2/ DEIMOS on 2006 November 19-21, using the "standard" DEIMOS setup with the $1200 \mathrm{l} / \mathrm{mm}$ grating, centered on $\sim 7500$ $\AA$. This central wavelength allowed coverage of the CaT at $\sim 8500-8700 \AA$, independent of slit position for GCs at the highest expected line-of-sight velocities in the NGC 1407 GC system $\left(\sim 2500 \mathrm{~km} \mathrm{~s}^{-1}\right)$, while capturing $\mathrm{H} \alpha$ for a reasonable fraction of the GCs observed. For this setup, our slit width of $1^{\prime \prime}$ gives an FWHM of $\sim 1.5 \AA\left(\sim 50 \mathrm{~km} \mathrm{~s}^{-1}\right.$ at the CaT $)$. The three masks were observed for 1.5-2 $\mathrm{hr}$ each in individual exposures of 30 minutes. The seeing on all three nights ranged from 0 '.55 to 0.8 . The large number of high-quality spectra that will we extract in Section 2.2 is testimony that the $\sim 16^{\prime} \times 5^{\prime}$ field of view of DEIMOS, along with its Flexure Compensation System, provide substantial improvements over Keck-1/LRIS $\left(\sim 8^{\prime} \times 6^{\prime}\right.$ field, not all of it usable for full spectral coverage).

\subsection{Keck/DEIMOS Data Reduction}

The spectra were reduced using the IDL spec2d pipeline written for the DEEP2 galaxy survey (J. A. Newman et al. 2009, in preparation). Images were flat-fielded using an internal quartz lamp, and wavelength-calibrated using ArKrNeXe arc lamps. After sky subtraction, individual one-dimensional spectra were optimally extracted. Some sample extracted spectra are shown in Figure 2.

Radial velocities were derived through cross-correlation around the $\mathrm{CaT}$ region with a library of standard stars spanning a wide range of spectral types (mid $\mathrm{F}$ to late $\mathrm{K}$ ) taken both on this run and during previous DEIMOS runs with the same setup. The derived velocities varied with spectral template choice by only $\sim 1-2 \mathrm{~km} \mathrm{~s}^{-1}$. In all cases the best-fit line-of-sight velocity 
Table 1

Photometry and Spectroscopy of Globular Cluster Candidates around NGC 1407

\begin{tabular}{|c|c|c|c|c|c|c|c|c|c|c|}
\hline ID & $\begin{array}{c}\text { R.A. } \\
(\mathrm{J} 2000)\end{array}$ & $\begin{array}{c}\text { Decl. } \\
(\mathrm{J} 2000)\end{array}$ & $\begin{array}{l}R_{\mathrm{p}} \\
\left({ }^{\prime \prime}\right)\end{array}$ & $\begin{array}{c}\text { P.A. } \\
\left({ }^{\circ}\right)\end{array}$ & $i_{0}^{\prime}$ & $\left(g^{\prime}-i^{\prime}\right)_{0}$ & $\left(r^{\prime}-i^{\prime}\right)_{0}$ & $\begin{array}{c}v_{\mathrm{p}} \\
\left(\mathrm{km} \mathrm{s}^{-1}\right)\end{array}$ & $\begin{array}{c}\Delta v_{\mathrm{p}} \\
\left(\mathrm{km} \mathrm{s}^{-1}\right)\end{array}$ & Type \\
\hline 70163 & $03: 40: 14.257$ & $-18: 41: 32.54$ & 406.1 & 175.1 & 22.94 & 0.77 & 0.22 & $0.178 ?$ & $\cdots$ & gal \\
\hline 71768 & $03: 40: 18.080$ & $-18: 41: 21.86$ & 403.9 & 167.3 & 21.93 & 1.08 & 0.36 & 1792 & 9 & GC \\
\hline 75581 & $03: 40: 08.063$ & $-18: 40: 55.52$ & 371.4 & 188.2 & 21.64 & 1.00 & 0.31 & 1750 & 13 & GC \\
\hline 81722 & 03:40:14.309 & $-18: 40: 10.38$ & 324.4 & 173.7 & 22.59 & 0.85 & 0.29 & 1439 & 13 & GC \\
\hline 82094 & $03: 40: 16.221$ & $-18: 40: 07.78$ & 326.0 & 168.9 & 22.38 & 1.24 & 0.45 & 1497 & 13 & GC \\
\hline 82266 & $03: 40: 15.476$ & $-18: 40: 06.01$ & 322.3 & 170.7 & 22.16 & 0.82 & 0.25 & 1686 & 17 & GC \\
\hline 84789 & $03: 40: 18.073$ & $-18: 39: 45.97$ & 311.1 & 163.4 & 22.35 & 1.16 & 0.39 & 1788 & 12 & GC \\
\hline 88899 & $03: 40: 26.549$ & $-18: 39: 13.77$ & 338.5 & 141.8 & 21.31 & 1.07 & 0.36 & 1524 & 7 & $\mathrm{GC}$ \\
\hline$\cdots$ & $\cdots$ & $\cdots$ & $\cdots$ & $\cdots$ & $\cdots$ & $\ldots$ & $\cdots$ & $\cdots$ & $\cdots$ & $\cdots$ \\
\hline$\ldots$ & $\cdots$ & $\cdots$ & $\cdots$ & $\cdots$ & $\cdots$ & $\cdots$ & $\cdots$ & $\cdots$ & $\cdots$ & $\ldots$ \\
\hline
\end{tabular}

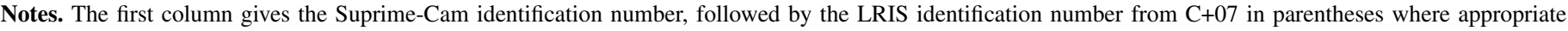

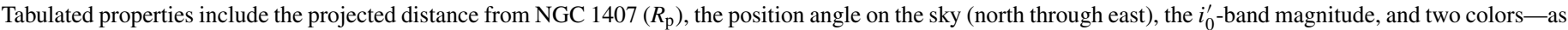

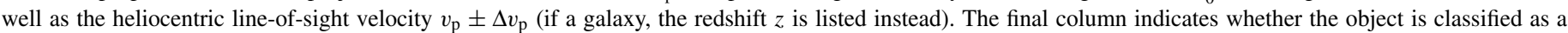

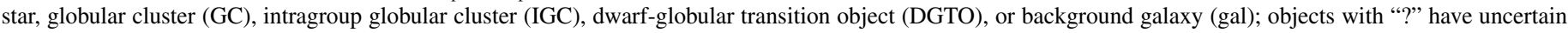

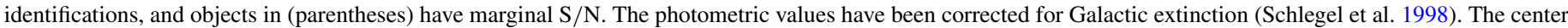
of the galaxy NGC 1407 is at 03:40:11.808 - 18:34:47.92 (J2000), and the systemic velocity is $1784 \mathrm{~km} \mathrm{~s}^{-1}$.

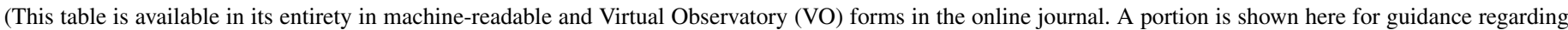
its form and content.)

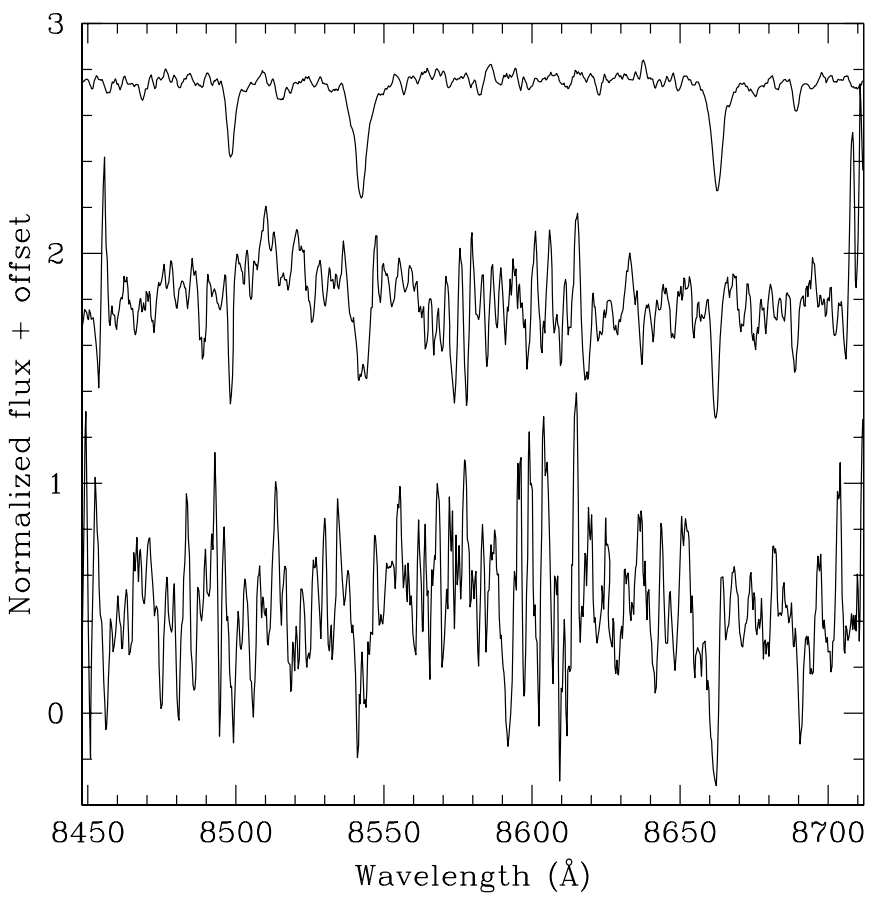

Figure 2. Extracted DEIMOS spectra of NGC 1407 GCs, shifted to zero redshift, with the $\mathrm{CaT}$ region shown, and with 5 pixel boxcar smoothing applied for visibility. The top spectrum shows a best-case example (ID 136205), the middle one is typical (ID 25581), and the lower one is a minimally acceptable example (ID 192009). The rest-frame wavelengths of the visible Ca II absorption lines are $8498 \AA, 8542 \AA$, and $8662 \AA$.

was verified by visual inspection of both the CaT region and $\mathrm{H} \alpha$ (where possible). Small (less than $5 \mathrm{~km} \mathrm{~s}^{-1}$ ) shifts in the zero-point velocities of the three masks were corrected using the location of the telluric $A$ band.

The velocity uncertainties were estimated from the width of the cross-correlation peak, and most fall within the range $\sim 5$ $15 \mathrm{~km} \mathrm{~s}^{-1}$, depending on the brightness of the GC. To assess the accuracy of these uncertainties, we deliberately included duplicate observations of GCs on different masks. In Figure 3, we show the results of the 10 repeated velocity measurements ( 9 GCs and 1 star). The velocities are nicely consistent within

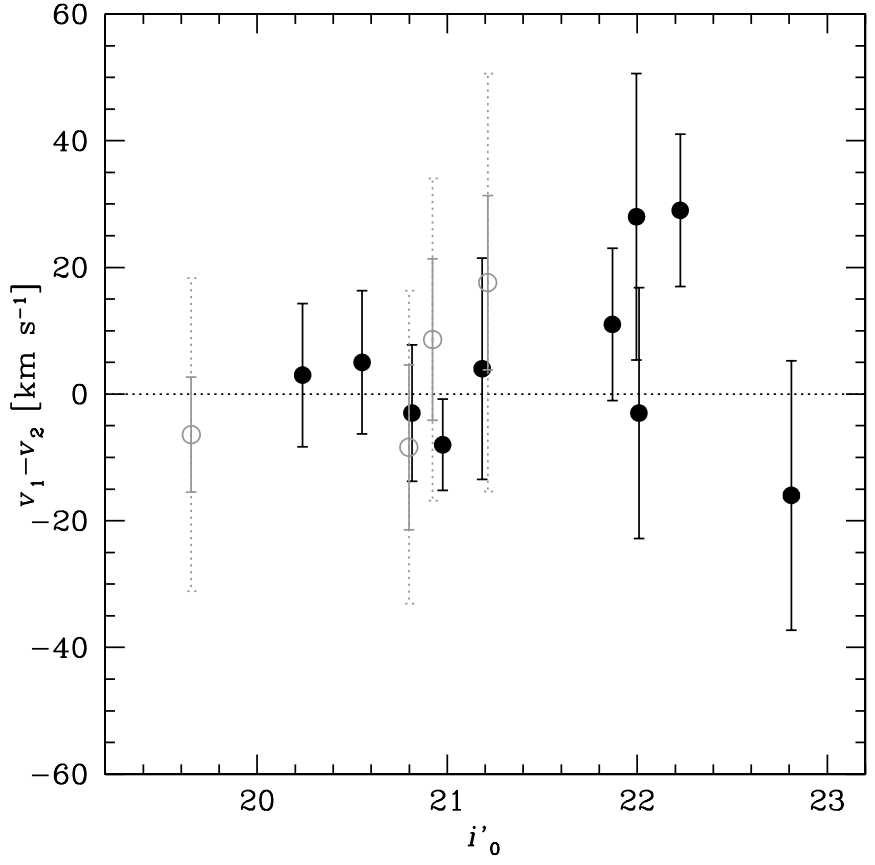

Figure 3. Comparisons of duplicate velocity measurements, as a function of $i_{0}^{\prime}$-band magnitude. The black filled circles show repeated measurements using DEIMOS. The gray open circles show duplicate measurements between LRIS and DEIMOS, where we have corrected for a mean offset of $11 \mathrm{~km} \mathrm{~s}^{-1}$. Error bars show the total estimated uncertainties. In the case of LRIS, the solid bars show statistical uncertainties, and the dotted bars include systematic uncertainties.

their stated uncertainties, to our faintest magnitude limit of $i_{0}^{\prime} \sim 23$.

Taking duplicates into account, we obtain velocities of 170 objects using DEIMOS, and list their properties in Table 1. We include two objects serendipitously obtained from mask alignment holes, and two faint blue objects that are borderline cases in our conservative spectral selection criteria. These latter two (No. 131881 and No. 160394) will be tentatively incorporated in our analyses, while checking that their inclusion does not affect any results. For a further 40 objects - generally the faintest in our masks-we could not obtain accurate line-of-sight ve- 
locities. Many of these showed peaks in the cross-correlation consistent with velocities expected for NGC 1407 GCs, but because of their low $\mathrm{S} / \mathrm{N}$ no clear visual confirmation of the CaT could be ascertained.

Of the good spectra, eight are clearly low-redshift galaxies (based on emission lines or extendedness), and another four are Galactic stars (see Section 3.1 for star/GC demarcations). There are $161 \mathrm{GC}$ candidates confirmed to be kinematically associated with NGC 1407, and since seven of the stars and galaxies had non-GC-like colors but were observed for lack of another target, our effective contamination rate was a mere $3 \%$. This high yield can be attributed to the richness of the NGC 1407 GCS, and to the excellent multicolor Suprime-Cam imaging and preselection.

One potential worry when observing the CaT is the flurry of bright sky lines in the wavelength vicinity. However, we have checked carefully that either $\mathrm{Ca} 2$ or $\mathrm{Ca} 3$ is always clear of a sky line (and usually $\mathrm{Ca} 1 \mathrm{also}$ ), except for narrow velocity ranges around $-150 \mathrm{~km} \mathrm{~s}^{-1},+225 \mathrm{~km} \mathrm{~s}^{-1}$, and $+1550 \mathrm{~km} \mathrm{~s}^{-1}$. The latter value may be of some concern to this study, as we may selectively lose a few faint GCs around this velocity despite the good sky subtraction of the DEIMOS pipeline.

\subsection{DEIMOS+LRIS Combined Sample}

To increase our sample size of GC velocities around NGC 1407, we include the 20 velocities obtained by $\mathrm{C}+07$ using Keck/LRIS. These LRIS velocities also allow us to make an external check on our DEIMOS velocities, using four GCs in common: see Figure 3. Given the stated LRIS systematic uncertainties, the duplicate measurements are fully consistent, although different instruments, telescopes, and reduction procedures were used. In fact, the systematic uncertainties of $\sim 20 \mathrm{~km} \mathrm{~s}^{-1}$ seem to be overly conservative, and we use only the statistical uncertainties, increasing them by $77 \%$ and subtracting $11 \mathrm{~km} \mathrm{~s}^{-1}$ from the velocities in order to match the DEIMOS data. The final LRIS uncertainties are typically $\sim 7 \mathrm{~km} \mathrm{~s}^{-1}$. In conclusion, the internal and external checks give us great confidence in the reliability of the Keck data. Velocity accuracies of $5-10 \mathrm{~km} \mathrm{~s}^{-1}$ are not essential for the current study of NGC 1407, but they do demonstrate that DEIMOS will be suitable for studying the dynamics of very low mass galaxies.

After combining duplicate measurements, we arrive at a sample of 190 GC candidate velocities, including 172 GCs and five other objects associated with NGC 1407 (see Section 3.1 for selection). These velocities, and associated source properties, are presented in Table 1.

\section{KINEMATICS RESULTS}

Having assembled a catalog of GC candidates, we select the probable GCs and examine their basic photometric and kinematical distributions in Section 3.1. We study the rotation, velocity dispersion, and kurtosis properties of the GCS in Sections 3.2, 3.3, and 3.4, respectively.

\subsection{GC Selection and Velocity Distribution}

As all of the GC candidates have colors within the expected range, we first select the GCs based on velocity. This selection is trivial, since there is a large gap between the lowest redshift obvious GC (1085 $\left.\mathrm{km} \mathrm{s}^{-1}\right)$ and the highest redshift obvious star (239 $\mathrm{km} \mathrm{s}^{-1}$; see Figure 4). We see no objects obviously associated with NGC 1400 (at a distance of $\sim 12^{\prime}$ and with a systemic velocity of $\sim 560 \mathrm{~km} \mathrm{~s}^{-1}$ ), although it is in principle possible for some of the low-velocity NGC 1407 GCs or the

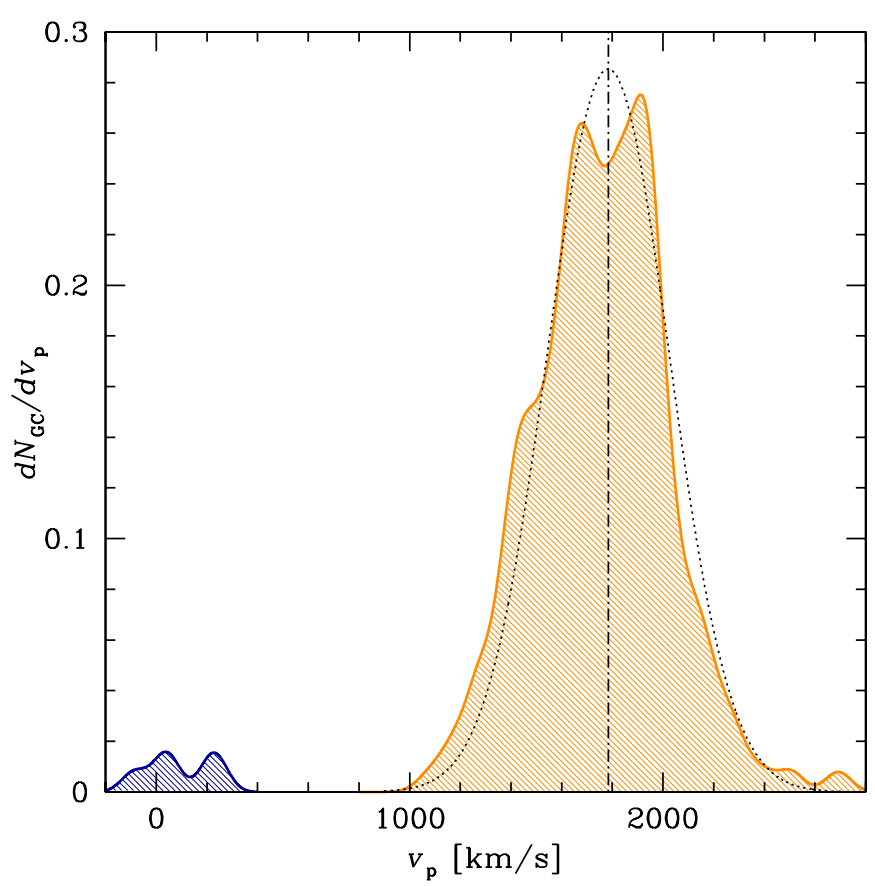

Figure 4. Distribution of GC candidates with velocity, smoothed by the measurement uncertainties and an additional $50 \mathrm{~km} \mathrm{~s}^{-1}$ for clarity. The dotdashed line marks the systemic velocity of NGC 1407. The dotted line shows a Gaussian with $\sigma_{\mathrm{p}}=241 \mathrm{~km} \mathrm{~s}^{-1}$.

(A color version of this figure is available in the online journal.)

"stars" to be extreme-velocity GCs belonging to NGC 1400. It is more probable that the density of NGC 1400 GCs is simply very low in our spectroscopically surveyed region (Figure 1 shows the photometrically estimated cross-over point between the GCS surface densities of NGC 1407 and NGC 1400), and further observations will be needed to determine the total radial extent of this galaxy's GCS.

We thus derive an initial sample of $177 \mathrm{GCs}$, including two cases that are borderline because of their low $\mathrm{S} / \mathrm{N}$. The colormagnitude diagram of the identified objects is shown in Figure 5, superimposed on the distribution of all sources in the region from the Suprime-Cam imaging. Our spectroscopy samples the GCs about 3 mag down the luminosity function, with almost equal numbers coming from the blue and the red subpopulations. The dividing color has been set at $\left(g^{\prime}-i^{\prime}\right)_{0}=0.98$ by inspection of the color-magnitude diagram. Assuming that the GCs are uniformly very old and that the color differences reflect metallicity differences (as $\mathrm{C}+07$ confirmed for a subsample of bright NGC 1407 GCs), we will in the rest of this paper refer to the blue and red GCs as "metal-poor" and "metal-rich," respectively. The GC color extrema and red/blue boundary of $\left(g^{\prime}-i^{\prime}\right)_{0}=(0.70,0.98,1.30)$ correspond to $[\mathrm{Fe} / \mathrm{H}] \sim$ $(-2.3,-1.1,+0.3)$ for a 12 Gyr age (Bruzual \& Charlot 2003), although the absolute values of these metallicities should not be considered certain.

An immediately apparent feature of the spectroscopic GC sample is a "blue tilt" of the metal-poor peak-a systematic trend of color with GC luminosity that has recently been discovered in many bright galaxies, including NGC 1407 (e.g., Strader et al. 2006; H+06a; Mieske et al. 2006; Spitler et al. 2008a; Harris 2009). This is now one of several detections of such a trend using ground-based photometry (see also Forte et al. 2007; Wehner et al. 2008), which argues against claims that the blue tilt is an artifact of HST photometry (Kundu 2008; Waters et al. 2009). 


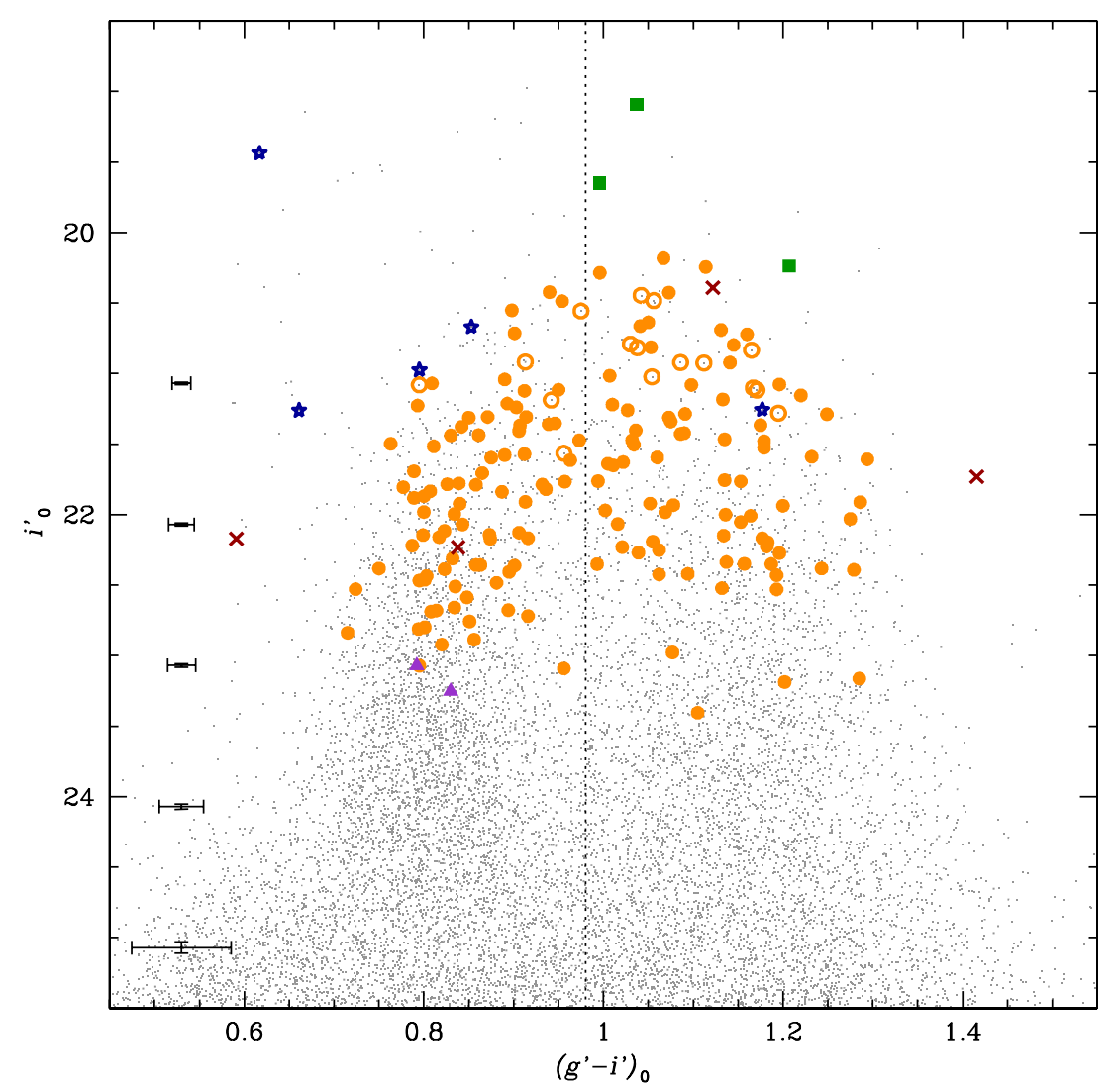

Figure 5. Color-magnitude diagram of GC candidates around NGC 1407. The symbols are as in Figure 1, except now the small gray points show GC candidates only within a $10^{\prime}$ radius of NGC 1407 . The typical photometric uncertainties are shown as error bars on the left. The dotted line shows the adopted boundary between metal-poor and metal-rich GCs: $\left(g^{\prime}-i^{\prime}\right)_{0}=0.98$.

(A color version of this figure is available in the online journal.)

Our data further support a convergence of the GC color distribution (including the metal-rich GCs) to a unimodal peak at high luminosities $(\mathrm{H}+06 \mathrm{a})$. The bright end of the GCLF is in many galaxies known to include extended objects such as $\omega$ Cen, G1 in M31, and ultra-compact dwarf galaxies (UCDs) more generally "dwarf-globular transition objects" (DGTOs; e.g., Hilker et al. 1999; Drinkwater et al. 2000; Haşegan et al. 2005; Evstigneeva et al. 2007; Wehner \& Harris 2007). As the nomenclature implies, some of these objects may have origins and properties that are distinct from "normal" GCs; e.g., the DGTOs could be the remnants of dwarf galaxies that were tidally stripped down to bare nuclei. It has been suggested that NGC 1407 harbors a sizable population of extended "GCs" $(\mathrm{H}+06 \mathrm{a})$, and it is important for our current study to ascertain whether our kinematics sample includes DGTOs, and whether their dynamics distinguishes them from the rest of the GCs.

We therefore re-examine the sizes of our candidate GCs in their original discovery images. The ACS data (near the galaxy's center) can discern the locus of GC sizes near $\sim 3-4 \mathrm{pc}$, and we do not support the conclusion of H+06a; their "extended" objects seem to be simply examples of bright GCs with normal sizes that are resolved at a distance of 20-30 Mpc. We can identify only one spectroscopic object that is a clear outlier (No. 136205 , with $R_{\text {eff }}=8 \mathrm{pc}$ ), which we designate as a DGTO. The Suprime-Cam data are much less well suited for measuring sizes without a very careful point-spread function (PSF)-based analysis. Again, there is one clear outlier (No. 160583), which was used for DEIMOS mask alignment but turned out to have a velocity corresponding to the NGC 1407 group. It is a known galaxy candidate named APMUKS(BJ) B033807.82-183957.5 (Maddox et al. 1990), and is well resolved in the Suprime-Cam images, with $R_{\text {eff }} \sim 25$ pc. Its color of $\left(g^{\prime}-i^{\prime}\right)_{0}=1.04$ and luminosity of $M_{i_{0}^{\prime}}=-12.5$ make this object likely to be a DGTO.

The two clear DGTOs around NGC 1407 are also the brightest two objects in our kinematics sample. They have somewhat red colors, and the second-ranked object was studied by $\mathrm{C}+07$, who found nothing unusual about its spectroscopic properties (age $\sim 11 \mathrm{Gyr},[\mathrm{Z} / \mathrm{H}] \sim-0.8,[\alpha / \mathrm{Fe}] \sim+0.3$ ). Another object, No. 134086, corresponds to the "galaxy" APMUKS(BJ) B033759.53-184331.2, but is unresolved in the ACS images, and we retain it as a GC. This gives us a first-pass sample of 175 GCs.

The metal-poor and metal-rich GCs are sampled fairly equally at all radii, which may be marginally inconsistent with the photometric inference that the metal-poor system is more radially extended; the inconsistency may be due to some hidden bias in the spectroscopic target selection. The distribution of confirmed GC colors is compared to the underlying distribution in Figure 6. The distributions are very similar, except for an excess of mildly metal-rich confirmed GCs that appears as a third color peak. This is probably an effect of the observational bias toward brighter objects, which show a convergence toward color unimodality (compare Figure 5) and which we will explore further in S+10. To a good approximation, we can conclude that the spectroscopically confirmed GCs are representative of the underlying GCS, although in Sections 3.2 and 3.3 we will double-check the kinematics for any magnitude dependence. 


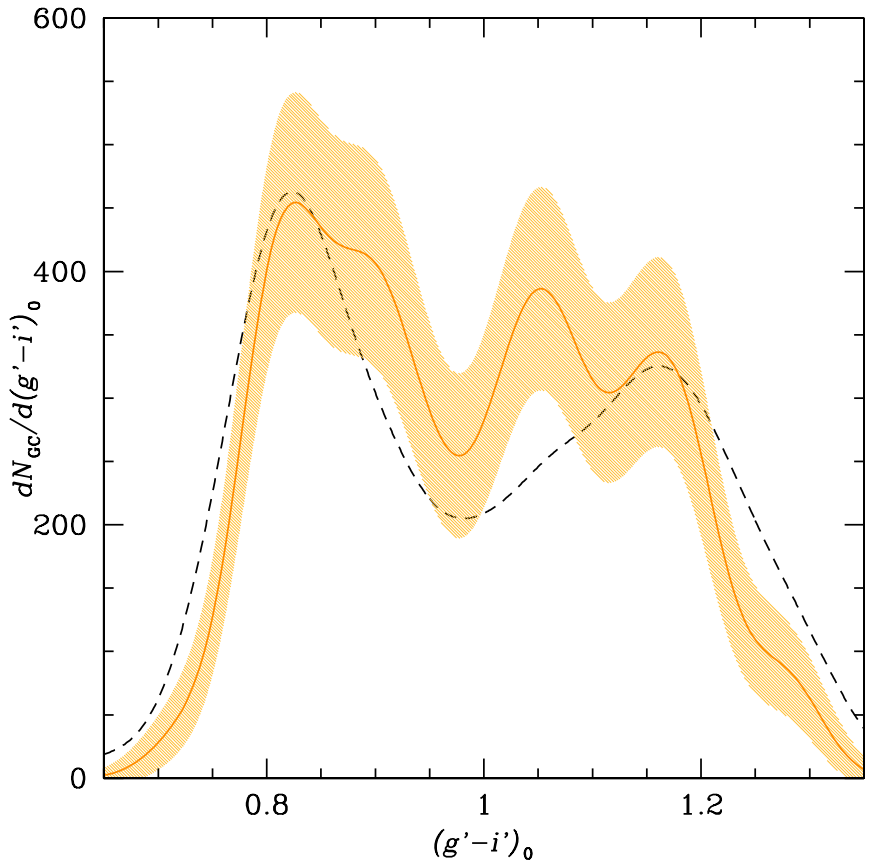

Figure 6. Color distribution of GCs around NGC 1407. The solid curve shows the 172 spectroscopically confirmed GCs, with shaded region indicating the approximate $1 \sigma$ uncertainties from Poisson noise. The dashed curve shows the underlying Suprime-Cam distribution of GC candidates with the same range of radius, colors, and magnitude, and renormalized to match the total number of confirmed GCs. For clarity, a smoothing kernel has been applied to these curves with a width of $\left(g^{\prime}-i^{\prime}\right)_{0}=0.03$, in addition to the measurement errors.

(A color version of this figure is available in the online journal.)

The azimuthal distribution of GC measurements is statistically uniform over the radial range of $106^{\prime \prime}-271^{\prime \prime}$. The mean and median values of the GC velocities in this annulus are 1769 and $1778 \mathrm{~km} \mathrm{~s}^{-1}$, respectively. The mean velocity of NGC 1407 from LRIS long-slit stellar kinematics is $1784 \mathrm{~km} \mathrm{~s}^{-1}$ (after subtracting the LRIS/DEIMOS systematic offset), which we adopt as the systemic velocity $v_{\text {sys }}$. For comparison, the $\mathrm{NED}^{7}$ velocity is $1779 \mathrm{~km} \mathrm{~s}^{-1}$.

The overall distribution of GC candidate velocities was shown in Figure 4. Given the uncertainties, the main peak of GCs is consistent with a simple Gaussian profile. We next inspect the velocity distribution with radius (Figure 7), seeing no obvious asymmetries.

There is one GC (No. 111003) with an "outlier" velocity (2692 $\mathrm{km} \mathrm{s}^{-1}$ ), well separated from the rest of the distribution. Calculating a smoothed profile of velocity dispersion with radius (see Section 3.3), we define a $3 \sigma$ envelope outside of which there is unlikely to be any object (if the distribution is Gaussian). The rogue GC is far outside of this envelope. It is a fairly bright, metal-poor object, which is borderline extended in the Suprime-Cam images, and whose velocity should be reliable. We conjecture that it belongs to either a loose intragroup population of GCs rather than to the GCS immediately surrounding NGC 1407 (compare the "vagrant" GC in the Fornax cluster; Schuberth et al. 2008) - or else to a sparse, dynamically distinct subpopulation of GCs that were propelled outward by three-body interactions, or were stripped from infalling satellites (e.g., Sales et al. 2007; Ludlow et al.

\footnotetext{
7 http://nedwww.ipac.caltech.edu/. The NASA/IPAC Extragalactic Database (NED) is operated by the Jet Propulsion Laboratory, California Institute of Technology, under contract with the National Aeronautics and Space Administration.
}

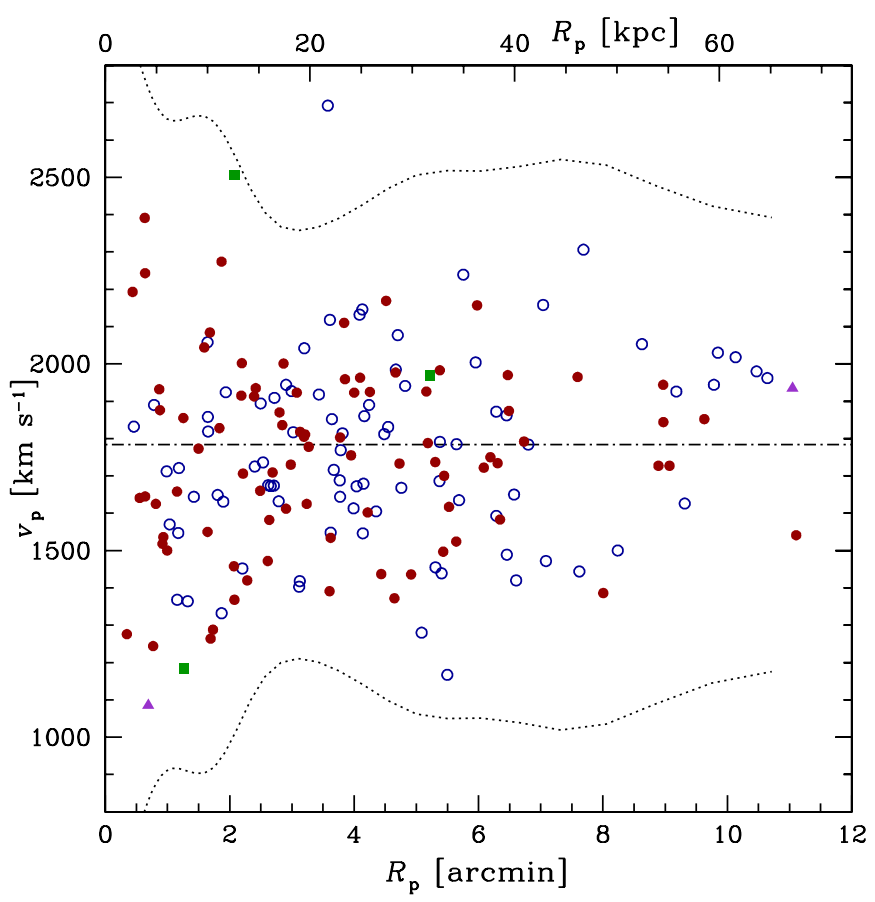

Figure 7. Velocity distribution of GC candidates with radius. The blue open circles are blue (metal-poor) GCs, and the red filled circles are red (metal-rich) GCs. The two purple filled triangles are uncertain (metal-poor) GC detections. The three DGTOs are shown by green squares. The systemic velocity is marked by a dot-dashed line, and the $\pm 3 \sigma$ "envelope" by dotted lines.

(A color version of this figure is available in the online journal.)

2009). This object is omitted from the following kinematical analyses. ${ }^{8}$

There is a second object (No. 135251, $2505 \mathrm{~km} \mathrm{~s}^{-1}$ ) that lies just below the upper $3 \sigma$ boundary. It is one of the brightest and reddest GC candidates around NGC 1407, appearing almost as an outlier in the color-magnitude diagram (Figure 5); from the Suprime-Cam images, it may be unusually extended. Noticing that one of the previously identified DGTOs also had an extreme (blueshifted) velocity, we suspect that there is a sizable population of unrecognized DGTOs concentrated toward the galactic center, with a high velocity dispersion. Indeed, a disproportionate share of the borderline extended objects seems to be red objects with high relative velocities, residing at small radii. Therefore, we assign this third object to the DGTO category, and keep in mind that the GCS kinematics inside $\sim 2^{\prime}-$ $3^{\prime}(\sim 15 \mathrm{kpc})$ may still be "contaminated" by a subpopulation of DGTOs (compare the case of NGC 1399, where a dozen DGTOs have been found within $\sim 15 \mathrm{kpc}$; Thomas et al. 2008).

We also note that No. 131881 has the lowest velocity in the sample $\left(1085 \mathrm{~km} \mathrm{~s}^{-1}\right)$, but we originally classified it as a borderline spectrum, so we remove it from our sample. Our final sample of GCs thus has 172 objects.

\subsection{Rotation}

As a rough measure of the amount of rotation in the NGC 1407 GCS, we perform a least-squares fit to the velocities as a function of azimuth, fitting the expression

$$
v(\theta)=v_{\mathrm{sys}}+v_{\mathrm{rot}} \sin \left(\theta_{0}-\theta\right)
$$

\footnotetext{
8 Neither this object nor any other GC candidate has a velocity that would place its pericenter near the virial radius as found in Section 4, which implies that they are all associated with the NGC 1407 group.
} 
Table 2

Kinematical Properties of NGC 1407 GCS in Various Subsamples

\begin{tabular}{|c|c|c|c|c|c|c|c|c|}
\hline Subsample & $N_{\mathrm{GC}}$ & $\begin{array}{c}v_{\text {rot }} \\
\left(\mathrm{km} \mathrm{s}^{-1}\right)\end{array}$ & $\begin{array}{l}\theta_{0} \\
\left(^{\circ}\right)\end{array}$ & Significance & $\begin{array}{c}\sigma_{\mathrm{p}} \\
\left(\mathrm{km} \mathrm{s}^{-1}\right)\end{array}$ & $\begin{array}{c}v_{\mathrm{rms}} \\
\left(\mathrm{km} \mathrm{s}^{-1}\right)\end{array}$ & $v_{\text {rot }} / \sigma_{\mathrm{p}}$ & $\kappa_{\mathrm{p}}$ \\
\hline \multicolumn{9}{|c|}{ All radii } \\
\hline All, 0'.35-11'.1 & 172 & $30_{-30}^{+20}$ & $47_{-47}^{+55}$ & $47 \%$ & $240_{-12}^{+14}$ & $241_{-12}^{+14}$ & $0.13_{-0.13}^{+0.08}$ & $-0.32 \pm 0.37$ \\
\hline Blue, $0.35-11^{\prime} .1$ & 86 & $41_{-41}^{+25}$ & $129_{-58}^{+49}$ & $44 \%$ & $232_{-16}^{+20}$ & $234_{-16}^{+21}$ & $0.18_{-0.18}^{+0.11}$ & $-0.32 \pm 0.51$ \\
\hline Red, $0.35-11^{\prime} .1$ & 86 & $58_{-42}^{+32}$ & $10_{-43}^{+34^{\circ}}$ & $68 \%$ & $243_{-16}^{+21}$ & $247_{-17}^{+21}$ & $0.24_{-0.18}^{+0.13}$ & $-0.28 \pm 0.51$ \\
\hline All, 0.35-1'.73 & 33 & $99_{-80}^{+62}$ & $-178_{-48}^{+37}$ & $\begin{array}{l}\text { Small radii } \\
57 \% \\
\text { Azimuthally complete }\end{array}$ & $291_{-30}^{+44}$ & $298_{-31}^{+45}$ & $0.34_{-0.28}^{+0.22}$ & $-0.61 \pm 0.80$ \\
\hline All, 1'.77-4'.52 & 75 & $79_{-37}^{+29}$ & $-12_{-25}^{+24}$ & $93 \%$ & $201_{-15}^{+19}$ & $209_{-15}^{+20}$ & $0.35_{-0.19}^{+0.15}$ & $-0.38 \pm 0.55$ \\
\hline Blue, $1^{\prime} .77-4.52$ & 38 & $12_{-12}^{+33}$ & $-115_{-98}^{+107}$ & $3 \%$ & $198_{-19}^{+27}$ & $198_{-19}^{+28}$ & $0.06_{-0.06}^{+0.17}$ & $-0.26 \pm 0.75$ \\
\hline Red, 1'.77-4'.52 & 37 & $149_{-49}^{+40}$ & $0_{-20}^{+1 \overline{7}^{-90}}$ & $98 \%$ & $190_{-19}^{+27}$ & $220_{-22}^{+31}$ & $0.79_{-0.28}^{+0.22}$ & $-0.41 \pm 0.76$ \\
\hline \multicolumn{9}{|c|}{ Large radii } \\
\hline All, $4.55-11^{\prime} .1$ & 64 & $71_{-55}^{+34}$ & $84_{-32}^{+44}$ & $74 \%$ & $237_{-19}^{+24}$ & $242_{-19}^{+25}$ & $0.30_{-0.23}^{+0.14}$ & $-0.36 \pm 0.59$ \\
\hline Blue, $4.55-11^{\prime} .1$ & 36 & $112_{-112}^{+47}$ & $102_{-33}^{+43}$ & $78 \%$ & $257_{-26}^{+36}$ & $272_{-28}^{+38}$ & $0.44_{-0.44}^{+0.19}$ & $-0.59 \pm 0.77$ \\
\hline Red, 4'.55-11'.1 & 28 & $61_{-61}^{+40}$ & $11_{-54}^{+46}$ & $47 \%$ & $194_{-22}^{+32}$ & $198_{-22}^{+33}$ & $0.32_{-0.32}^{+0.21}$ & $-0.24 \pm 0.86$ \\
\hline \multicolumn{9}{|c|}{ Outside center } \\
\hline All, 1'.77-11'.1 & 139 & $52_{-30}^{+26}$ & $32 \pm 29$ & $83 \%$ & $222_{-12}^{+15}$ & $225_{-13}^{+15}$ & $0.23_{-0.13}^{+0.12}$ & $-0.34 \pm 0.41$ \\
\hline Blue, 1'.77-11'.1 & 74 & $54_{-54}^{+29}$ & $122_{-45}^{+43}$ & $62 \%$ & $233_{-17}^{+22}$ & $237_{-18}^{+22}$ & $0.23_{-0.23}^{+0.13}$ & $-0.32 \pm 0.55$ \\
\hline Red, 1'.77-11'.1 & 65 & $115_{-38}^{+32}$ & $2_{-19}^{+17}$ & $99 \%$ & $194_{-15}^{+20}$ & $211_{-17}^{+21}$ & $0.59_{-0.21}^{+0.17}$ & $-0.39 \pm 0.59$ \\
\hline
\end{tabular}

where $v_{\text {rot }}$ is the rotation amplitude and $\theta_{0}$ is the direction of the angular momentum vector. The physical interpretation of this model is discussed in Côté et al. (2001, hereafter $\mathrm{C}+01$; note the different sign convention). $\mathrm{C}+01$ and some other studies have used error-weighted least-squares rotation fitting (equivalent to $\chi^{2}$ fitting). However, this approach is not so appropriate for a system with an intrinsic dispersion, and it is preferable to use straightforward least-squares fitting (equivalent to minimizing the rotation-subtracted dispersion). The differences become important when the true underlying rotation is weak $\left(v_{\text {rot }} / \sigma_{\mathrm{p}} \lesssim 0.4\right)$. Further refinements are possible (e.g., Kissler-Patig \& Gebhardt 1998; Woodley et al. 2007), but for now we will keep things simple using leastsquares fitting. The procedures we describe below for evaluating statistical uncertainties and significance follow Sharples et al. (1998).

Using the full data set of $172 \mathrm{GCs}$, we find $v_{\text {rot }}=$ $30_{-30}^{+20} \mathrm{~km} \mathrm{~s}^{-1}$ and $\theta_{0}=47_{-47}^{+55} \mathrm{deg}$, where the uncertainties have been estimated through Monte Carlo simulated recovery of the best-fit models. An additional test with random reshuffling of the GC data point position angles shows a $53 \%$ probability of a rotation amplitude this high happening just by chance, i.e., there is no significant rotation detected. The position angle is close to the galaxy's major axis (P.A. $\simeq 55-65 \mathrm{deg} ; \mathrm{S}+08$; $\mathrm{S}+10$ ), implying that the GCS may rotate around rather than along the major axis.

Before drawing any conclusions, we must recognize that the rotation field can be complicated by a mixture of metal-poor and metal-rich GCs with very different rotation patterns, by variations with radius, and by potential DGTO contamination in the innermost regions. Therefore, we fit the rotation for different subsamples of metallicity and radius, as summarized in Table 2 . The most secure result is for the azimuthally complete region of $R_{\mathrm{p}}=1.77-4.52(11-27 \mathrm{kpc})$, where there is highly significant rotation close to the major axis, produced by the metal-rich GCs (see Figure 8). At larger radii, there is not a clear rotation signature for the metal-rich GCs, but the metal-poor GCs appear to rotate around an orthogonal direction intermediate to the major and minor axes. The combination of the misaligned metal-rich and metal-poor rotation vectors outside the center

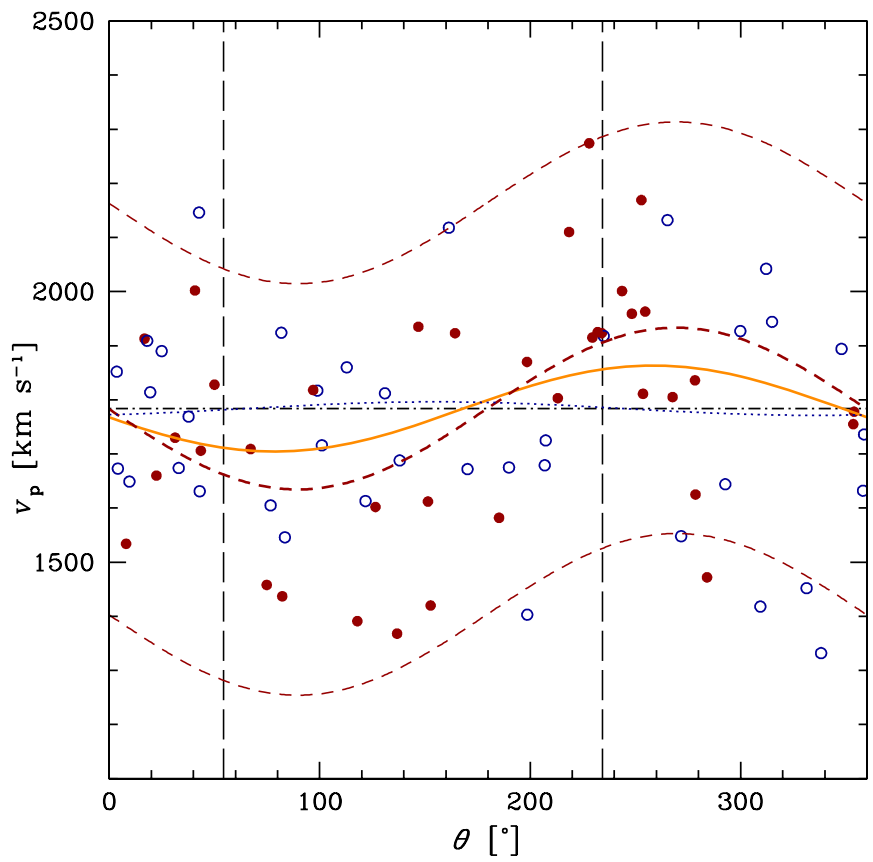

Figure 8. Rotation of NGC 1407 GCS, illustrated by velocities plotted against position angles. The symbols are as in Figure 7, with GCs in only the azimuthally complete region (1'.77-4.52) plotted. The best-fit sine curves are shown for all GCs (solid), metal-poor GCs (dotted), and metal-rich GCs (dashed). For illustration, the $\pm 2 \sigma_{\mathrm{p}}$ boundaries of the metal-rich GCs are also shown, corresponding to the dispersion of this subsample. The horizontal line shows the systemic velocity, and the vertical lines mark the galaxy photometric major axis $\left(\sim 55^{\circ}\right.$ at $\left.3^{\prime} ; \mathrm{S}+10\right)$.

(A color version of this figure is available in the online journal.)

produces a possible overall rotation around the major axis (with $v_{\text {rot }} / \sigma_{\mathrm{p}} \sim 0.1$; see Section 3.3 for the calculation of the velocity dispersion $\sigma_{\mathrm{p}}$ ). Looking in more detail at the kinematics, the rotation of both subpopulations is driven by the region $R_{\mathrm{p}} \sim 2^{\prime}-$ $6^{\prime}(13-38 \mathrm{kpc})$.

In addition, we find evidence for a cold moving group of metal-poor GCs at $\sim 10^{\prime}(\sim 60 \mathrm{kpc})$ to the northeast, with $v_{\text {rot }}=224_{-12}^{+13} \mathrm{~km} \mathrm{~s}^{-1}$ and $\sigma_{\mathrm{p}}=14_{-6}^{+10} \mathrm{~km} \mathrm{~s}^{-1}$ (this feature 
is visible in Figure 7). These GCs have a wide range of luminosities but a relatively narrow spread in color $\left[\left(g^{\prime}-i^{\prime}\right)_{0}=\right.$ $0.89 \pm 0.06]$, suggesting a shared formational history. One speculative interpretation is that the cold group traces the recent disruption of a disk galaxy (compare Feldmann et al. 2008).

We have further investigated dependencies of rotation on GC luminosity, and while it at first glance appears that the brighter GCs rotate more strongly and with a different axis than the faint GCs, on closer inspection, this may be simply due to spatial biasing with magnitude of our selected GCs. The reintroduction of the DGTO velocities (see Section 3.1) would change some of the rotation vectors noticeably. Given the focus of this paper on the mass analysis, we will defer closer examinations of rotational properties to a future paper with a more complete data set.

We would like to make one final comparison, between the rotation of the GCs and the field stars in NGC 1407. The general hypothesis is that the metal-poor GCs in galaxies are closely related to their metal-poor halo stars (which are observationally inaccessible at the distance of NGC 1407), while the metal-rich GCs are related to their old metal-rich bulge stars (which correspond to the bulk of the diffuse starlight in an old elliptical like NGC 1407; see Pritzl et al. 2005; Forte et al. 2007; Baumgardt et al. 2008). Unfortunately, the existing stellar kinematics data in NGC 1407 extend to only $\sim 40^{\prime \prime}$ along the major axis $(\mathrm{S}+08)$, which is the region with only minimal numbers of measured GC velocities, and furthermore with possible DGTO contamination. Comparing the stellar and GC rotation fields will thus require further efforts, either by extracting galaxy rotation directly from the same DEIMOS slits used for the GCs (compare Norris et al. 2008; Proctor et al. 2008, $2009)$, or by observing planetary nebulae (PNe) as proxies for metal-rich halo stars (Romanowsky 2006). We do note that the field stars and metal-rich GCs in the overlap region were found by Spolaor et al. (2008b) to have similar ages, metallicities, and $\alpha$-element enhancements. If the metal-rich GCs are directly associated with the field stars, then the increase of $v_{\text {rot }}$ from $\sim 50 \mathrm{~km} \mathrm{~s}^{-1}$ in the central parts $(\mathrm{S}+08)$ to $\sim 100 \mathrm{~km} \mathrm{~s}^{-1}$ in the outer parts may support the hypothesis that the "missing" angular momenta of elliptical galaxies (e.g., Fall 1983) can be found in their halos (e.g., Weil \& Hernquist 1996; B+05).

\subsection{Velocity Dispersion}

We construct a projected GCS velocity dispersion profile from these data out to a distance of $60 \mathrm{kpc}$ from the central galaxy. Technically, we compute the rms velocity profile, $v_{\text {rms }} \sim \sqrt{\Sigma_{i}\left(v_{i}-v_{\text {sys }}\right)^{2} / N}$, which includes the (small) contribution from rotation, but we will refer to it freely as the "velocity dispersion." The true dispersion $\sigma_{\mathrm{p}}$ is also calculated by subtracting the contribution from the rotation (Section 3.2), and both $v_{\text {rms }}$ and $\sigma_{\mathrm{p}}$ are shown in Table 2. We compute the binned dispersions and its uncertainties using standard formulae (Danese et al. 1980), and complementarily construct smoothed dispersion radial profiles using maximum likelihood fitting to a Gaussian line-of-sight velocity distribution, weighted at each point by neighboring data (see Bergond et al. 2006). We also use this maximum likelihood technique to fit power-law functions to the dispersion profiles,

$$
v_{\mathrm{rms}}^{2}\left(R_{\mathrm{p}}\right)=v_{\mathrm{rms}, 0}^{2} \times\left(R_{\mathrm{p}} / R_{0}\right)^{-\gamma_{\mathrm{p}}} .
$$

With the full data set of 172 GCs, there is a striking transition in the dispersion profile (Figure 9) from a rapid decline inside $1: 8(11 \mathrm{kpc}$ - confirming the finding of $\mathrm{C}+07)$ to a fairly constant

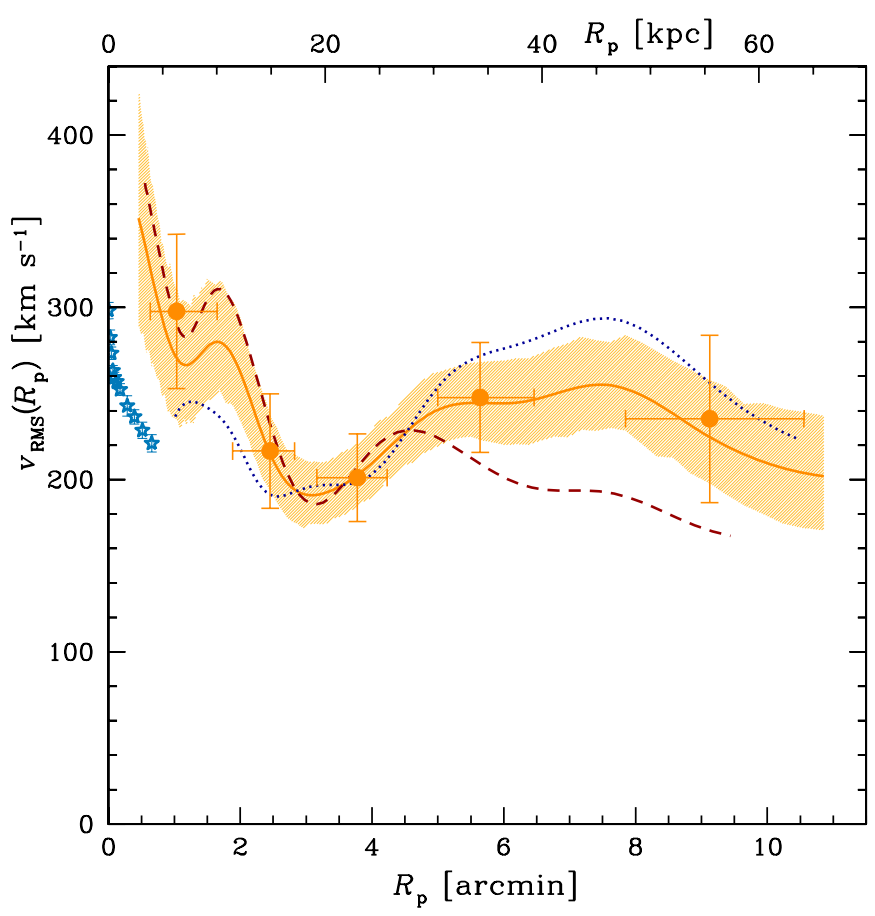

Figure 9. Projected rms velocity profile of NGC 1407. The circles with error bars show binned GCS values with no color selection. The solid curve shows a smoothed GCS profile using a variable-width Gaussian kernel (see Bergond et al. 2006 for details). The shaded region indicates the 1- $\sigma$ uncertainty boundaries for the full GCS sample, as computed by Monte Carlo simulations. The dotted and dashed lines show the profiles for the metal-poor and metal-rich GC subsamples, respectively. The star symbols with error bars show long-slit stellar kinematics data from S+08, as updated in Proctor et al. (2009).

(A color version of this figure is available in the online journal.)

value outside of this radius $\left(\gamma_{\mathrm{p}}=-0.07 \pm 0.26\right)$. There is also the hint of another decline outside of 7.5 $(50 \mathrm{kpc})$, but it is not significant. The constancy of the dispersion profile out to $\sim 10 R_{\text {eff }}$ is immediately a strong indication of a massive DM halo around NGC 1407.

To better understand the dispersion profile, we analyze the metal-rich and metal-poor GCs separately (Figure 9). The dispersions of the metal-rich and metal-poor clusters are similar over the range of $R_{\mathrm{p}} \sim 3^{\prime}-4.5(\sim 20-30 \mathrm{kpc})$. Inside this range, the metal-rich GCs have a significantly higher dispersion than the metal-poor GCs, and at larger radii, their dispersion is lower. This difference was already visible by eye in Figure 7, and translates into very different slopes with radius, with $\gamma_{\mathrm{p}}=-0.26 \pm 0.23$ (increasing) for the metal-poor GCs, and $\gamma_{\mathrm{p}}=+0.54 \pm 0.19$ (decreasing) for the metal-rich GCs.

Such a difference in dispersion slope is qualitatively expected from the differences in spatial density profiles of the two subpopulations (the blue GCS is more extended than the red GCS), but explaining this situation quantitatively may be a challenge (investigated in Section 4.1.4). The steepness of the inner slope (driven by the metal-rich GCs) would have been even steeper if the probable DGTOs had been left in the sample (see Section 3.1), which we surmise may still include contamination from a population of as yet unresolved, metal-rich DGTOs on fast orbits at small galactocentric radii (we will return to this issue in Section 5.1). Within the uncertainties of our limited data set, we do not find any trend of dispersion with GC luminosity.

Since we are unsure about the nature of this steep inner slope, we will omit the inner dispersion data point from our mass profile analyses (Section 4.1), assuming that it is affected by a 
distinct subpopulation of objects with very different orbits than the rest of the GCs. Note that the dispersion of the metal-rich GCs is inconsistent with the field stars' dispersion in the narrow region of overlap (Figure 9).

\subsection{Velocity Kurtosis}

Other measures of line-of-sight velocity distribution (LOSVD) beyond rotation and dispersion can provide key information about the orbital structure. In particular, measures of departures of the LOSVD from a Gaussian shape, such as provided by the fourth-order velocity moment, are important diagnostics that can be crudely used to distinguish between radial and tangential orbits. Here, we compute a basic projected reduced kurtosis statistic that is approximated by

$$
\kappa_{\mathrm{p}}=\left[\frac{1}{N} \sum_{i=1}^{i=N} \frac{\left(v_{i}-v_{\mathrm{sys}}\right)^{4}}{v_{\mathrm{rms}}^{4}}\right]-3 \pm \sqrt{\frac{24}{N}},
$$

with a more complicated bias-corrected expression for smallnumber statistics given by $G_{2}$ in Joanes \& Gill (1998). ${ }^{9}$ For a Gaussian LOSVD, $\kappa_{\mathrm{p}}=0$, which describes isotropic orbits in a logarithmic potential. The kurtosis is only useful for regions over which $v_{\text {rms }}$ is approximately constant, so we exclude the inner regions of the GCS $\left(R_{\mathrm{p}}<1.73\right)$ from our analysis.

For the overall GCS outside the central regions, we find $\kappa_{\mathrm{p}}=-0.30 \pm 0.41$, i.e., a suggestion of negative kurtosis. The "flat-topped" behavior of the platykurtic LOSVD can be marginally seen by eye in Figures 4 and 7, where the velocities do not peak strongly around $v_{\text {sys }}$ (particularly at large radii). The LOSVD is still statistically consistent with being Gaussian (the kurtosis estimator is unfortunately not a very powerful measure of LOSVD shape), but a strongly peaked (leptokurtic) distribution does appear to be ruled out, with important implications for the orbits as we will see in the next section. For the metal-poor GCs, we find $\kappa_{\mathrm{p}}=-0.24 \pm 0.55$, and for the metal-rich GCs, we find $\kappa_{\mathrm{p}}=-0.30 \pm 0.59$, so there is no overall difference detected in the LOSVD shapes between the two subsystems.

We would like to further consider the behavior with radius $\kappa_{\mathrm{p}}\left(R_{\mathrm{p}}\right)$ but the data are inadequate for detecting significant trends. We do see signs of the kurtosis becoming stronger with GC luminosity: see Figure 10, where there appears to be a dearth of velocities near $v_{\text {sys }}$ for $i_{0}^{\prime} \lesssim 21.3$. This effect is driven by bright, central objects, and does not impact the result of negative kurtosis outside the central regions.

One caveat about our entire kurtosis analysis is that a Gaussian LOSVD was assumed in Section 3.1 to remove outliers; for example, restoring No. 111003 to the data set would imply a higher $v_{\text {rms }}$ and a significantly positive $\kappa_{\mathrm{p}}$. More robust measures of LOSVD shapes and outlier removal will eventually require detailed self-consistent dynamical modeling. We will return to the dynamical implications of the kurtosis in Section 4.1.4, and to the issue of the luminosity dependence in Section 5.1.

We also note that there are no faint GCs $\left(i_{0}^{\prime}>22.5\right)$ with $v \quad 1550 \mathrm{~km} \mathrm{~s}^{-1}$, which may be due to systematic loss near this velocity from sky line collisions (Section 2.2). But even if this is the case, we would expect to have lost only about one

\footnotetext{
9 In principle, $\kappa_{\mathrm{p}}$ can be compromised by velocity measurement uncertainties, rotation, and small-number statistics. However, we have modeled these effects analytically and with Monte Carlo simulations, and determined that they are negligible for this data set, except for the small number statistics which may bias the results by $\sim-0.1$.
}

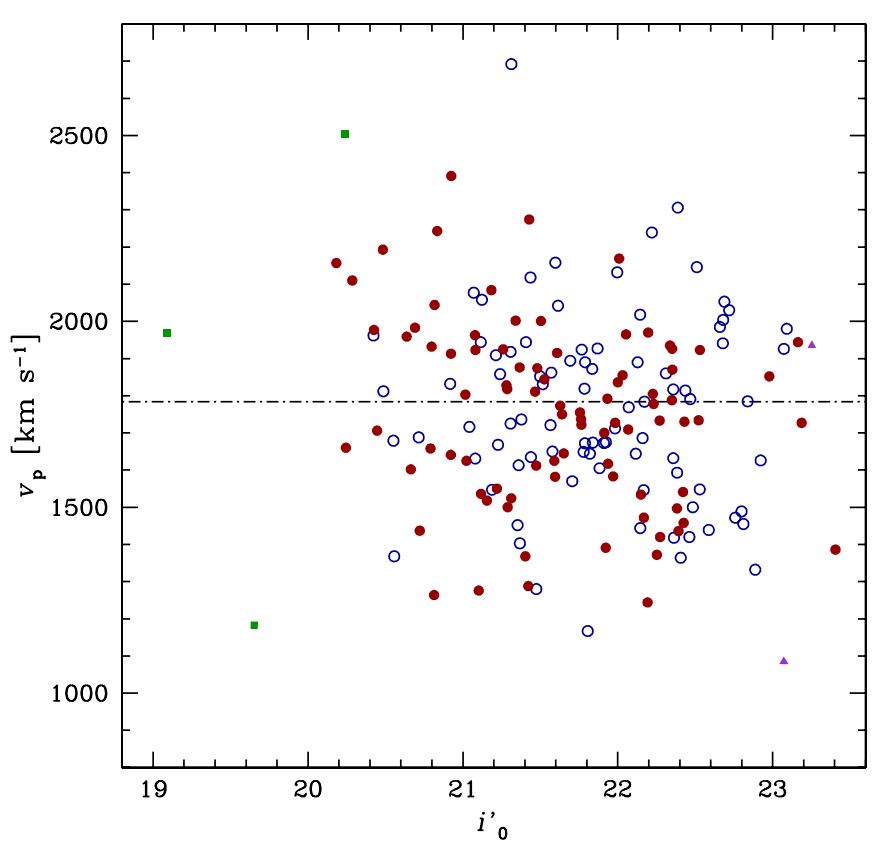

Figure 10. Distribution of GC candidate velocities vs. magnitude. Symbols are as in Figure 7. Note the paucity of bright GCs near the systemic velocity.

(A color version of this figure is available in the online journal.)

GC to the effect, which should not compromise our results. Additionally, a few GC candidates near the extreme mask edges experienced wavelength-dependent vignetting that was not recognized during the original mask design. However, we have verified that this issue would not have caused any velocity bias in the recovered GC spectra.

\section{NGC 1407 MASS PROFILE}

Reaching the main focus of our paper, we consider various aspects of the mass profile of the NGC 1407 system. In Section 4.1, we model the dynamics of the GCS to derive a set of permitted mass models. In Section 4.2, we present an X-ray-based mass analysis, and in Section 4.3, we compare independent mass constraints in NGC 1407 and in other galaxies.

\subsection{GCS Dynamics}

Here, we model the dynamics of the GCs around NGC 1407, beginning with a summary of the modeling assumptions in Section 4.1.1, then results with no DM in Section 4.1.2, and a $\Lambda$ CDM-motivated model in Section 4.1.3. Alternative models that better reproduce the data are explored in Section 4.1.4.

\subsubsection{Modeling Assumptions}

Our approach is to construct a sequence of cosmologically motivated galaxy+halo mass models, calculate their projected GCS rms velocity profiles $v_{\text {rms }}\left(R_{\mathrm{p}}\right)$, and compare these predictions to the data. For this initial dynamical model of the GCS of NGC 1407, we make several simplifying assumptions. First is that the system is spherically symmetric. The NGC 1407 outer galaxy isophotes have an ellipticity of $e \sim 0.05$ (S+08), supporting sphericity as a reasonable approximation, which seems to be the case in general for massive ellipticals at group and cluster centers, although flattening may become important at radii of $\gtrsim 20 \mathrm{kpc}$ (Porter et al. 1991; Ryden 1996).

Second, we assume a particular form for the GCS velocity dispersion tensor that is nearly isotropic near the central galaxy, 
and becomes increasingly radially anisotropic at larger galactocentric radii (details below). We also assume that the system is in dynamical equilibrium and that the solutions to the Jeans equations are physical and stable (these assumptions are supported by analyses of simulated DM halos with no recent major merger; Diemand et al. 2005, hereafter D+05).

Third, we represent the cumulative mass profile $M(r)$ by a constant mass-to-light ratio $\left(\Upsilon_{*}\right)$ Sérsic (1968) model for the galaxy's field stars, plus a DM halo characterized by a NavarroFrenk-White (NFW) profile, expressed as a circular velocity that is independent of distance and bandpass:

$$
\begin{aligned}
v_{\mathrm{c}}^{2}(r) \equiv & \frac{G M(r)}{r} \\
= & \frac{G \Upsilon_{*} L_{*}}{r} \times\left\{1-\frac{\Gamma\left[(3-p) m,\left(r / a_{\mathrm{S}}\right)^{1 / m}\right]}{\Gamma[(3-p) m]}\right\} \\
& +v_{s}^{2}\left[\frac{r_{s}}{r} \ln \left(1+r / r_{s}\right)-\frac{1}{1+r / r_{s}}\right],
\end{aligned}
$$

where $m$ and $a_{\mathrm{S}}$ are the Sérsic index and scale radius, and $p$ is a function of $m$ (Mamon \& Łokas 2005b, hereafter MŁ05), while $v_{s}$ and $r_{s}$ are the characteristic velocity and spatial scales for the DM halo (Navarro et al. 1996). The $v_{\mathrm{c}}$ profile of the halo reaches a maximum of $0.465 v_{s}$ at a radius of $2.16 r_{s}$.

Our Sérsic stellar mass model is obtained by fitting a preliminary combined photometric profile (I-band ACS $+i^{\prime}$ band Suprime-Cam) outside of a radius of $1^{\prime \prime}$, resulting in fit parameters corresponding to $\left(m=4.32, R_{\text {eff }}=57^{\prime \prime}=5.8 \mathrm{kpc}\right.$, $\left.M_{I}=-23.3\right)$. As is typical for giant ellipticals, these values are fairly uncertain (total luminosity by at least $20 \%$, and $R_{\text {eff }}$ by at least $30 \%$ ) because of the large fraction of light contained in the faint stellar halo. One obtains a far superior fit to the galaxy light using a Nuker model (Lauer et al. 1995) or a core+Sérsic model (e.g., Trujillo et al. 2004), but the former does not converge to a finite luminosity, and the latter is not simple to incorporate in the modeling equations. At the radii corresponding to our GC velocity measurements (to $\sim 10^{\prime}$ ), the total luminosity of the Sérsic model deviates from that of the core+Sérsic model by up to $15 \%$, but as we will see, the total mass in this region is dominated by DM, and in any case, the luminosity profile is not well constrained outside $2^{\prime}$.

The galaxy mass profile is uniquely determined by the stellar mass-to-light ratio $\Upsilon_{*}$, which we estimate from a stellar populations analysis $(\mathrm{Z}+07)$ to be $\Upsilon_{*, I}=2.12 \Upsilon_{\odot, I}\left(\Upsilon_{*, B}=\right.$ $4.45 \Upsilon_{\odot, B}$ ), assuming a Kroupa intial mass function (IMF). ${ }^{10}$ The adopted NGC 1407 galaxy mass is thus $M_{*}=2.0 \times$ $10^{11} M_{\odot}$; we will examine the impact of $\Upsilon_{*}$ on our results in Sections 4.1.4 and 4.3.1.

The density profile for the GCS is obtained by a Sérsic model fit to preliminary surface density data $\Sigma_{\mathrm{GC}}\left(R_{\mathrm{p}}\right)$ from Suprime-Cam, over the radial range of $0.8-23^{\prime}(\mathrm{S}+10)$. As shown in Figure 11, the fit is characterized by the parameters $\left(m=1.39 \pm 0.09, R_{\text {eff }}=3.52 \pm 0.07=21 \mathrm{kpc}\right)$, demonstrating that the radial extent of the GCS of NGC 1407 is far

\footnotetext{
10 This value for $\Upsilon_{*}$ may seem low relative to historical findings in other bright ellipticals (e.g., $\Upsilon_{*, I} \sim 3 \Upsilon_{\odot, I}$ in Cappellari et al. 2006, and $\Upsilon_{*, B} \sim 6$ $\Upsilon_{\odot, B}$ in Gerhard et al. 2001), and for NGC 1407 in particular, the stellar populations models of Humphrey et al. (2006, hereafter $\mathrm{H}+06 \mathrm{~b})$ imply $\Upsilon_{*, B}=5.7 \pm 0.5 \Upsilon_{\odot, B}$. However, the $\mathrm{Z}+07$ value is meant to characterize the stars outside $0.5 R_{\text {eff }}$ rather than in the oft-studied central regions $\left(\sim 0.1 R_{\text {eff }}\right.$ in the case of $\mathrm{H}+06 \mathrm{~b})$; another study averaging over $1 R_{\mathrm{eff}}$ found $\Upsilon_{*, B} \sim 4$ for NGC 1407 (Tortora et al. 2009). The full range of possible values Z+07 found for different radii and modeling assumptions was $\Upsilon_{*, B}=3.8-7.1 \Upsilon_{\odot, B}$ for a Kroupa IMF, and up to $11.2 \Upsilon_{\odot, B}$ for a Salpeter IMF.
}

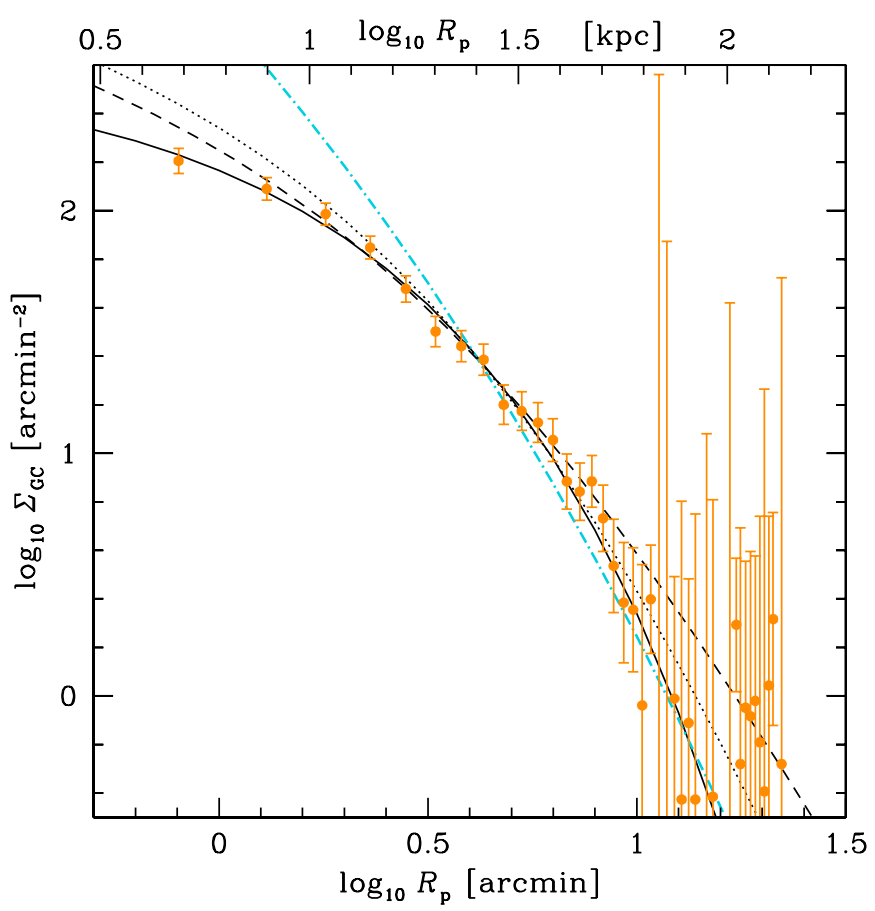

Figure 11. NGC 1407 GCS surface density profile. The points with error bars show Suprime-Cam data with a background contamination level subtracted $(\mathrm{S}+10)$. The solid curve is our best-fit Sérsic model. For comparison, the NGC 1407 galaxy light model is shown as a blue dot-dashed line (with arbitrary normalization). The other curves show profiles corresponding to the $\Lambda \mathrm{CDM}$ simulations of D+05, for overdensities of $2.5 \sigma$ (dashed) and $4 \sigma$ (dotted).

(A color version of this figure is available in the online journal.)

greater than that of its field starlight. Dividing the GCS into subpopulations, the metal-poor component is more extended than the metal-rich, with $\left(m \sim 1.3, R_{\text {eff }} \sim 4.3\right)$ and $\left(m \sim 1.2, R_{\text {eff }}\right.$ $\sim 2^{\prime} .8$ ), respectively. We have also verified that these density fits are fair representations of the bright GC subpopulation probed in our kinematics sample $\left(i_{0}^{\prime}<23\right)$. The uncertainty in the GCS density profile is not explicitly included in our dynamical modeling, but we will explore the effects of reasonable variations in this profile as needed.

The model GCS dispersion profile $v_{\mathrm{rms}}\left(R_{\mathrm{p}}\right)$ is calculated with the spherical Jeans equations as summarized in MŁ05. ${ }^{11}$ In using these equations, we are also assuming that the net rotation can be simply folded into $v_{\text {rms }}$ along with the velocity dispersion, since the observed rotation in NGC 1407 is not dynamically important $\left(v_{\text {rot }} / \sigma_{\mathrm{p}} \sim 0.4\right.$; see Section 3.2$)$. We generally attempt to fit the dispersion data outside of 1.8 only, since the central values may be skewed by DGTO contamination (see Section 3.3). We will return to this assumption in Section 4.1.4.

\subsubsection{Constant Mass-to-Light Ratio Models}

Our first model is a galaxy with no DM halo, or a "constant$\Upsilon$ " model; see Table 3 for the details of the main models that we will discuss. In this case, we keep things simple by adopting an isotropic velocity dispersion tensor: $\beta(r)=0$, where $\beta \equiv 1-\sigma_{\theta}^{2} / \sigma_{r}^{2}$ specifies the bias of specific kinetic energy in the tangential direction $\sigma_{\theta}^{2}$ relative to the radial direction $\sigma_{r}^{2}$. This model's predicted $v_{\text {rms }}\left(R_{\mathrm{p}}\right)$ for the GCs in the outer parts is much lower than the observed values (see model $\mathrm{S}$ in Figure 12,

\footnotetext{
11 We use the Prugniel \& Simien (1997) function $p(m)$ in the GCS density profile since in this case it appears to provide a better match than the Lima Neto et al. (1999) function to the Sérsic surface density profile.
} 
Table 3

Summary of Noteworthy Mass Models for the NGC 1407 Group

\begin{tabular}{|c|c|c|c|c|c|c|c|c|c|c|c|}
\hline Label & $\begin{array}{c}\Upsilon_{*} \\
{\left[\Upsilon_{\odot, B}\right]}\end{array}$ & $\beta(r)$ & $\begin{array}{c}v_{s} \\
\left(\mathrm{~km} \mathrm{~s}^{-1}\right)\end{array}$ & $\begin{array}{c}\rho_{s} \\
\left(10^{6} M_{\odot} \mathrm{kpc}^{-3}\right)\end{array}$ & $\begin{array}{c}r_{s} \\
(\mathrm{kpc})\end{array}$ & $\begin{array}{c}r_{\mathrm{vir}} \\
(\mathrm{Mpc})\end{array}$ & $c_{\mathrm{vir}}$ & $\begin{array}{c}M_{\mathrm{vir}} \\
\left(10^{13} M_{\odot}\right)\end{array}$ & $\begin{array}{c}\tilde{\chi}_{\mathrm{GC}}^{2} \\
\left(N_{\mathrm{dof}}\right)\end{array}$ & $\begin{array}{l}\tilde{\chi}_{\mathrm{X}-\text { ray }}^{2} \\
\left(N_{\text {dof }}\right)\end{array}$ & Model description \\
\hline $\mathrm{S}$ & 4.45 & 0 & $\ldots$ & $\ldots$ & $\ldots$ & 0.18 & $\ldots$ & 0.03 & $43.5(4)$ & $46.0(7)$ & Constant- $\Upsilon$ \\
\hline GR & 4.45 & Equation (6) & $1600_{-400}^{+600}$ & $0.83_{-0.50}^{+1.23}$ & $230_{-110}^{+290}$ & $1.35_{-0.37}^{+0.65}$ & $5.8_{-2.0}^{+2.7}$ & $14.2_{-8.9}^{+31.9}$ & $2.6(2)$ & $10.9(7)$ & Radial $\Lambda \mathrm{CDM}$ \\
\hline GI & 4.45 & 0 & $1300_{-200}^{+600}$ & $1.2_{-0.8}^{+1.7^{+50}}$ & $170_{-80}^{+200}$ & $1.13_{-0.28}^{+0.37}$ & $6.7_{-2.3}^{+3.1}$ & $8.4_{-5.0}^{+17.7}$ & $1.1(2)$ & $11.1(7)$ & Isotropic $\Lambda$ CDM \\
\hline II & $\ldots$ & 0 & $\ldots$ & $\cdots$ & $\ldots$ & $\ldots$ & $\ldots$ & $\ldots$ & $0.8(2)$ & $81.7(7)$ & Isotropic GC inversion \\
\hline GT & 4.45 & -0.5 & $1200_{-200}^{+400}$ & $1.5_{-1.0}^{+2.1}$ & $140_{-70}^{+160}$ & $1.00_{-0.24}^{+0.45}$ & $7.3_{-2.5}^{+3.4}$ & $5.7_{-3.2}^{+12.1}$ & $0.8(2)$ & $11.6(7)$ & Tangential $\Lambda \mathrm{CDM}$ \\
\hline XR & 4.45 & Equation (6) & $1300 \pm 100$ & $11_{-3}^{+1.0}$ & $52_{-9}^{+13}$ & $0.88_{-0.06}^{+0.09}$ & $17 \pm 2$ & $3.9_{-0.9}^{+1.3}$ & $8.4(4)$ & $0.8(5)$ & X-ray-based NFW \\
\hline XT & $\ldots$ & -0.5 & $\ldots$ & $\ldots$ & $\cdots$ & $0.76_{-0.04}^{+0.44}$ & $\ldots$ & $2.6^{+7.5}$ & $5.6(4)$ & $0.0(2)$ & Tangential X-ray based \\
\hline$X G$ & $\ldots$ & Figure 13 & $\ldots$ & $\ldots$ & $\ldots$ & $0.76_{-0.04}^{+0.04}$ & $\ldots$ & $2.6_{-0.5}^{-7.5}$ & $0.7(4)$ & $0.0(2)$ & GC/X-ray consonance \\
\hline $\mathrm{C}$ & 4.45 & -0.5 & $1300 \pm 200$ & $2.4_{-0.9}^{+1.8}$ & $120 \pm 40$ & $1.07_{-0.17}^{+0.18}$ & $9.1_{-17}^{+2.3}$ & $7.1_{-28}^{+4.5}$ & $4.0(2)$ & $7.2(5)$ & Consensus $\Lambda \mathrm{CDM}$ \\
\hline GG & 4.45 & -0.5 & $1400_{-100}^{+200}$ & $0.71_{-0.28}^{+0.90}$ & $230_{-70}^{+100}$ & $1.26 \pm 0.21$ & $5.4_{-1.1}^{+1.6}$ & $11.5_{-4.7}^{+6.8}$ & $0.7(2)$ & $14.0(7)$ & GCs + galaxies \\
\hline
\end{tabular}

Notes. The characteristic NFW halo density is $\rho_{s} \equiv v_{s}^{2} /\left(4 \pi G r_{s}^{2}\right)$. The reduced $\chi^{2}$ statistic, $\tilde{\chi}^{2} \equiv \chi^{2} / N_{\text {dof }}$, is provided along with the number of degrees of freedom $N_{\text {dof. }}$.

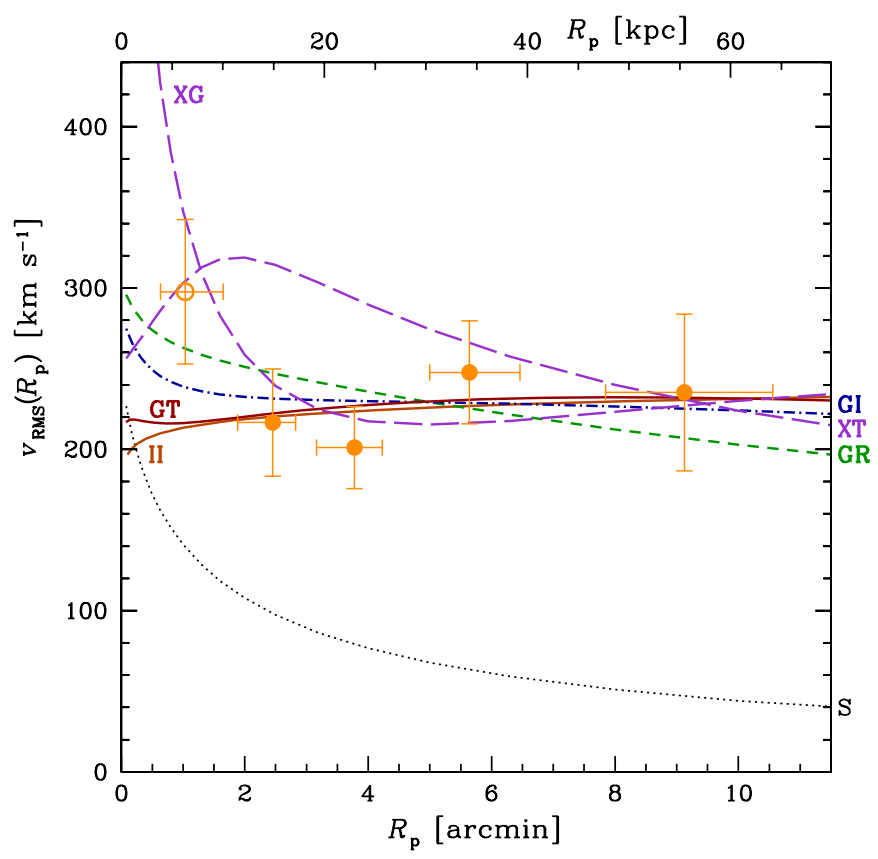

Figure 12. NGC 1407 GCS projected rms velocity profile. The points with error bars show the measurements, while curves show model predictions that are described in more detail in the main text. The black dotted curve (S) shows a stars-only galaxy (no DM). The green short-dashed curve (GR) is a model with a $\Lambda \mathrm{CDM}$ halo and a theoretically motivated GC anisotropy profile (Equation (6)). The dark blue dot-short-dashed curve (GI) is a similar model with an isotropic GC system. The orange solid curve (II) is a power-law fit to the data. The red solid curve (GT) is a $\Lambda$ CDM halo with tangential anisotropy. The purple longdashed curves come from the X-ray-based mass model: with either $\beta=-0.5$ (XT) or an anisotropy that varies from $\beta=1$ in the center to $\beta=-\infty$ at large radii (XG; see Figure 13).

(A color version of this figure is available in the online journal.)

with comparison data corresponding to the $v_{\mathrm{rms}}\left(R_{\mathrm{p}}\right)$ profile from Section 3.3). In principle, the data could be roughly fitted by setting the distance to $40 \mathrm{Mpc}$, the stellar mass-to-light ratio to $\Upsilon_{*, I} \sim 7 \Upsilon_{\odot, I}\left(\Upsilon_{*, B} \sim 15 \Upsilon_{\odot, B}\right)$, and the outer GC orbits to nearly circular $(\beta \sim-10)$, but this solution is highly contrived and implausible.

The discrepancy between the velocities measured and predicted for a simple stellar galaxy is strong evidence for either nonclassical gravity behavior (e.g., Brownstein \& Moffat 2006; Angus et al. 2008), or for an unseen mass component-or for both. It is beyond the scope of this paper to treat alternative gravities, so we will simply presume to be witnessing the effects of a massive DM halo. Note that the missing mass is not attributable to the hot gas in the system, whose mass of $\sim 10^{10} M_{\odot}(\mathrm{Z}+07)$ is less important than even the stellar component.

\subsubsection{Fiducial $\Lambda C D M$ Models with Anisotropy}

We next examine a fiducial set of models including DM halos. These models are parameterized by the virial mass $M_{\mathrm{vir}}$ and the halo concentration $c_{\text {vir }} \equiv r_{\text {vir }} / r_{s}$, which are correlated according to the predictions of $N$-body cosmological simulations by the relation:

$$
c_{\mathrm{vir}}=17.88 \times\left(M_{\mathrm{vir}} / 10^{11} M_{\odot}\right)^{-0.125},
$$

with a scatter in $c_{\text {vir }}$ (at fixed $M_{\text {vir }}$ ) of 0.14 dex (though the scatter may be smaller for high-mass, relaxed halos; Bullock et al. 2001; Wechsler et al. 2002; see Section 3.2 in Napolitano et al. 2005). Here, we have adopted a virial overdensity of $\Delta_{\text {vir }}=101$, a power-spectrum normalization of $\sigma_{8}=0.9$, and a Hubble constant of $H_{0}=70 \mathrm{~km} \mathrm{~s}^{-1} \mathrm{Mpc}^{-1}$. The relation (5) was derived from Bullock et al. (2001) for galaxy-mass halos, but turns out to also be valid for higher masses, differing by at most $5 \%$ from the updated relation of Neto et al. (2007) over the range $M_{\text {vir }}=(2-70) \times 10^{13} M_{\odot}$. Lower values for $\sigma_{8}$ would imply systematically lower concentrations, but the effect is probably smaller than the intrinsic scatter of the concentrations (Duffy et al. 2008).

For simplicity, we do not include any back-reaction of the baryons on the DM profile (such as adiabatic contraction: e.g., Blumenthal et al. 1986; Gnedin et al. 2004), which would be only a small correction at the outer DM-dominated radii that we are probing (see also $\mathrm{H}+06 \mathrm{~b}$ ). We also do not include the most recent refinements to the NFW profile used in Equation (4) (e.g., Navarro et al. 2004; Gao et al. 2008).

The anisotropy profile is motivated by numerous theoretical studies that generically predict that DM halos, and the baryonic tracers within them, are radially anisotropic in their outer regions (e.g., van Albada 1982; Cole \& Lacey 1996; Diaferio 1999; Colín et al. 2000; Fukushige \& Makino 2001; Vesperini et al. 2003; Rasia et al. 2004; Wojtak et al. 2005; Dekel et al. 2005; MŁ05; D+05; Abadi et al. 2006; Hansen \& Moore 2006; Sommer-Larsen 2006; Nipoti et al. 2006; Oñorbe et al. 2007; McMillan et al. 2007; Prieto \& Gnedin 2008). D+05 in particular explored the dependence of the anisotropy of tracers 
on their concentrations within their host halos, and we adopt their Equation (8) to represent the anisotropy profile of the NGC $1407 \mathrm{GCS}^{12}$ :

$$
\beta(r)=\beta_{0} \frac{r}{r+r_{0}} .
$$

To assign values to the asymptotic anisotropy $\beta_{0}$ and the break radius $r_{0}$, we first compare our GCS surface density profile to the parameterized projected profiles of the D+05 simulations corresponding to DM subsets characterized by an overdensity of $n$ times the rms background fluctuation (see their Equation (1)). We ignore the central cusp in these density profiles since D+05 did not report the natural variation around a typical log slope of -1.2 , and since the central GCS number density may be altered considerably by destruction processes. Focusing on the outer density profiles, we find that $n \sim 4$ is needed to reproduce the steep decline seen in NGC 1407 (see Figure 11). The data include both metal-rich and metal-poor GCs, so this fit is not appropriate for determining GC formation redshift as proposed by D+05 and Moore et al. (2006), but rather provides a heuristic reasonable assumption for the anisotropy profile. The $\log$ density slope at $r_{0}$ of the $n=4$ simulation model is -3.3 , and finding the location in our GCS density model with the same slope, we arrive at $r_{0}=6.33=38.5 \mathrm{kpc}$. As specified by D+05 Equation (8), $n=4$ corresponds to $\beta_{0}=0.8$; Figure 13 shows the resulting anisotropy profile. The values of $\beta_{0}$ and $r_{0}$ are not very sensitive to our choice for $n$.

Now to find the best-fit fiducial model, we combine a $\chi^{2}$ fit of the model to the $v_{\text {rms }}\left(R_{\mathrm{p}}\right)$ data (excluding the innermost point which we regard as questionable, see Sections 3.1 and 3.3) with a $\chi^{2}$ fit to Equation (5) including its scatter. This fitting method is equivalent to a Bayesian analysis where the model priors are given by Equation (5). We find a virial mass $M_{\text {vir }} \quad 1.4 \times 10^{14} M_{\odot}$, with a corresponding virial radius of $r_{\mathrm{vir}}=1.0-2.0 \mathrm{Mpc}$-which is somewhat larger than the optical size of the group of $0.4-0.6 \mathrm{Mpc}(\mathrm{B}+06 \mathrm{~b})$. As seen in Figure 12 (with best fit reported in Table 3 as model GR), this "standard" $\Lambda$ CDM model is roughly consistent with the GCS data (including the central $v_{\text {rms }}$ point) but it does not match particularly well the constant $v_{\text {rms }}$ profile outside of the center $\left(-0.3 \lesssim \gamma_{\mathrm{p}} \lesssim+0.2\right)$. The model predicts a fairly steep decline of $v_{\text {rms }}\left(R_{\mathrm{p}}\right)$ (slope $\left.\gamma_{\mathrm{p}} \sim+0.3\right)$, despite a rising $v_{\mathrm{c}}(r)$. This behavior is due partially to the strong radial anisotropy, and partially to the very low Sérsic index of the GCS density profile, whose flat core and sharp outer edge mimic the effects of radial anisotropy.

\subsubsection{Model Alternatives}

Suspecting that at least one of the modeling assumptions is awry, we next try excluding any a priori constraint on the concentration. Without this constraint, there is a severe degeneracy between the virial quantities $M_{\mathrm{vir}}$ and $c_{\mathrm{vir}}$, since even with an NFW profile assumed, we cannot make reliable extrapolations from data extending to only $\sim 5 \%$ of $r_{\mathrm{vir}}$. As seen in the left panel of Figure 14, the DM halo could have a high mass and a low concentration, or alternatively a low mass and a high concentration. The right panel illustrates some physical quantities more directly constrained by the data: the circular velocity inside $10^{\prime}$, and the characteristic acceleration of the halo $a_{s} \equiv 0.193 v_{\mathrm{s}}^{2} / r_{s}$ (which corresponds to a constraint on the projected mass density $\rho_{s} r_{s}$, or equivalently to the

\footnotetext{
12 The D+05 anisotropy profile has the rotational component subtracted, unlike our modeling convention, but this difference impacts their predictions at the level of $\Delta \beta \sim-0.02$ at most.
}

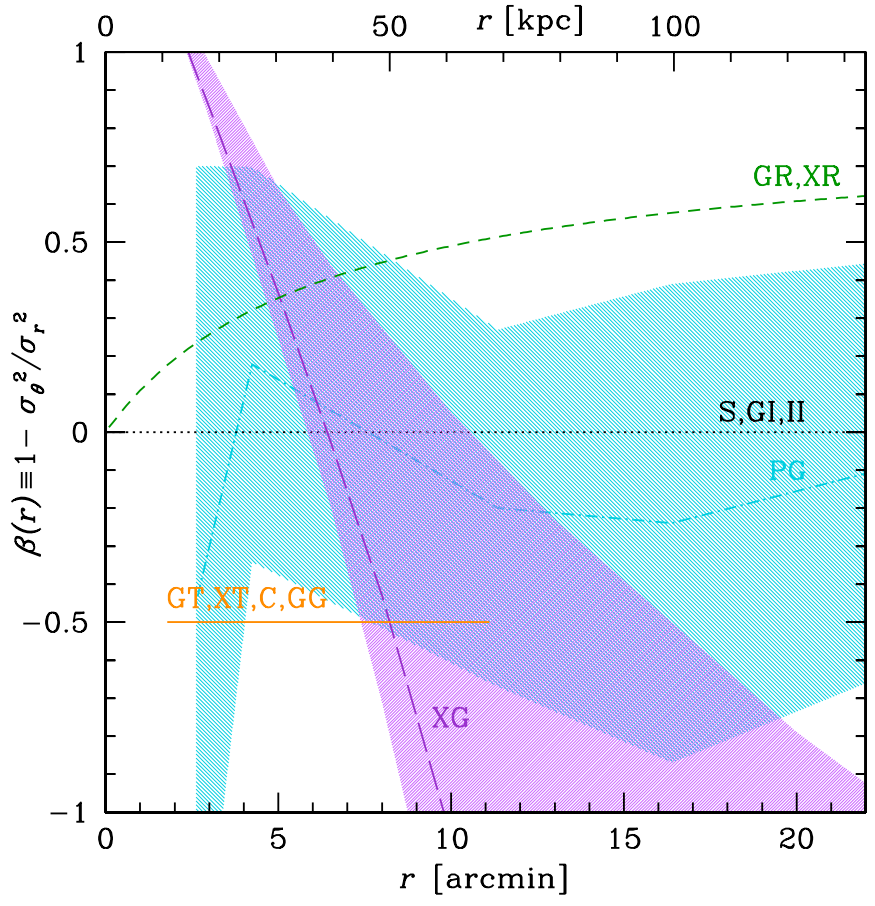

Figure 13. Velocity dispersion anisotropy profiles for GCs in NGC 1407. The black horizontal dotted line shows isotropy. The green short-dashed line shows a theoretically motivated profile (Equation (6)). The orange horizontal solid line shows the dynamics-based estimate of the GC anisotropy (Section 4.1.4). The purple long-dashed line with surrounded shaded region shows the profile necessary for agreement with the X-ray analysis (Section 4.3.2). The blue dotdashed line with shaded region shows the cosmological simulations of metalpoor GCs from Prieto \& Gnedin (2008, see Section 5.1), scaled to the virial radius of NGC 1407.

(A color version of this figure is available in the online journal.)

gravitational lensing convergence $\kappa$ ); these quantities are more tightly constrained than other halo parameterizations (e.g., Alam et al. 2002). The GC data naturally prefer very lowconcentration, high-mass halos in order to try to reproduce the flat $v_{\text {rms }}\left(R_{\mathrm{p}}\right)$ profile despite the low Sérsic index and the presence of radial anisotropy. The best-fit solution is implausible $\left(M_{\text {vir }} \sim 10^{17} M_{\odot}, c_{\text {vir }} \sim 10^{-2}\right)$, and the fit is still not very good $\left(\chi_{\mathrm{GC}}^{2}=3.0\right.$ for 2 dof $)$.

Another possibility is that our adopted $\beta(r)$ profile is too extreme, and so we return to the standard $c_{\mathrm{vir}}-M_{\mathrm{vir}}$ correlation, with a simple $\beta=0$ isotropic assumption. There is little theoretical justification for isotropy on scales of $\sim 50 \mathrm{kpc}$, but there are empirical indications of isotropic GC systems in other massive group-central ellipticals (e.g., Romanowsky \& Kochanek 2001, hereafter RK01; C+01; C+03; Samurović \& Ćirković 2008; Johnson et al. 2009, hereafter J+09; see summary in Hwang et al. 2008, hereafter H+08). With isotropy, we find a better fit to the data, with naturally a lower $M_{\text {vir }}$ preferred since mass is no longer "hidden" by radial anisotropy (see model GI in Table 3 and in Figures 12 and 14). The resulting virial radius of $0.8-1.7 \mathrm{Mpc}$ is closer to the optical group radius.

Given that the isotropic model provides an improved fit, we use this simplifying fact as a starting point to derive an estimated mass profile directly from the data, using no theoretical priors such as Equation (4). We wish to deproject the $v_{\text {rms }}\left(R_{\mathrm{p}}\right)$ profile and use it in the isotropic Jeans equation to find the $v_{\mathrm{c}}(r)$ profile. We start with the standard Abel deprojection equation for velocity dispersion (e.g., Binney \& Tremaine 1987, Equation (4-58b)) and differentiate it, integrating by parts and 

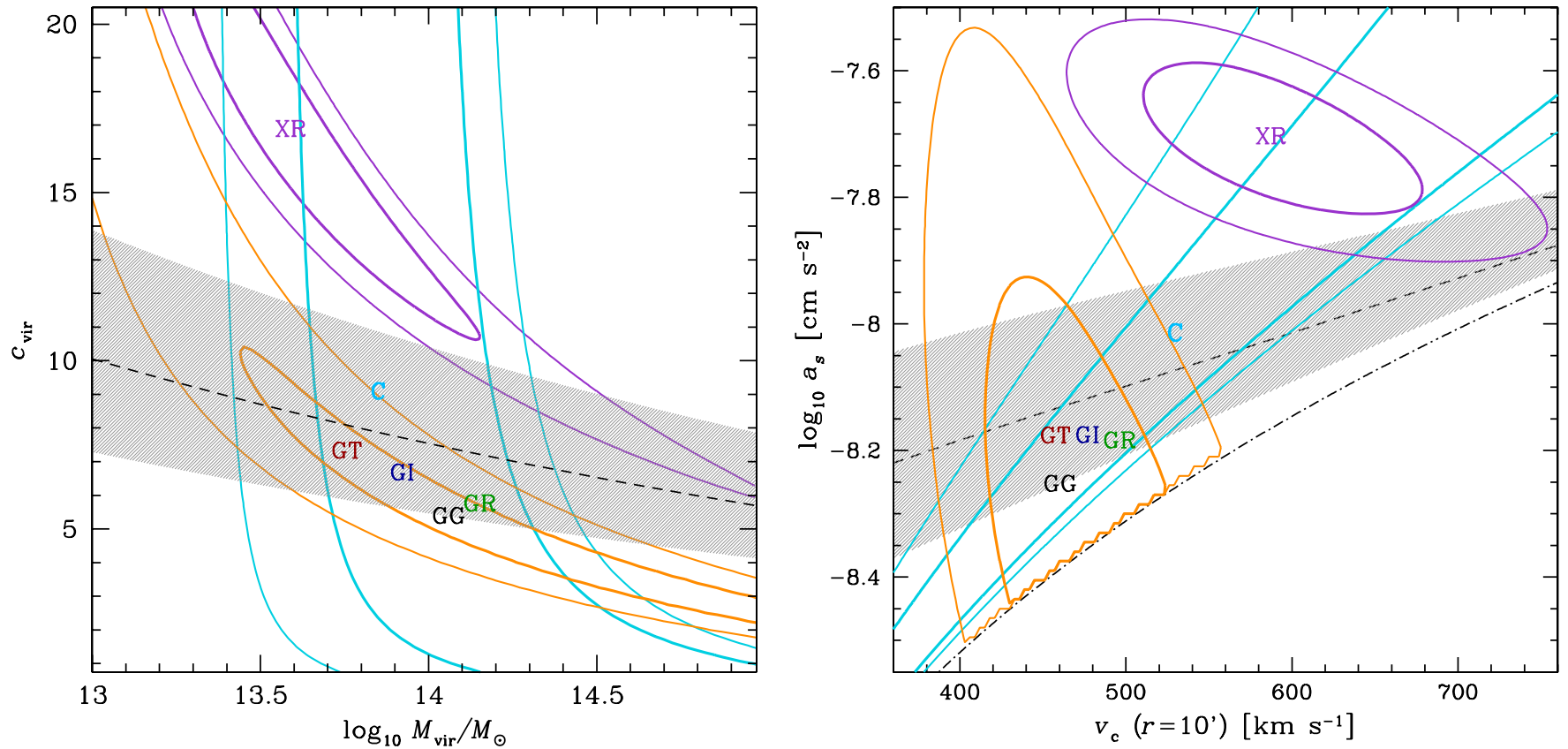

Figure 14. Mass profile solutions for NGC 1407. Thick and thin solid contours show the $1 \sigma$ and $2 \sigma$ confidence levels, respectively (as found by $\Delta \chi^{2}$ tests); none of the contoured solutions include a concentration prior. The orange contours toward the bottom of the panels correspond to the tangentially anisotropic GCS solutions, the purple contours toward the top to the X-ray solutions (model XR), and the blue near-vertical contours to the group galaxies solutions. The mean relation and scatter from cosmological simulations are shown by the dashed curve and shaded region (see the text). Additional letters label various best-fit solutions as described in the text and in Table 3. The left panel shows the virial mass and concentration, and the right panel shows the circular velocity inside the GC-constrained region of $10^{\prime}$ $(60 \mathrm{kpc})$ as well as the characteristic halo acceleration. The dot-dashed line in the right panel shows the physically meaningful limit of the NFW model (i.e., $\left.c_{\mathrm{vir}}=0\right)$. The plots demonstrate that the GCS dynamics are consistent with a "normal" high-mass $\Lambda$ CDM halo, while the X-ray-based mass model is mildly inconsistent with $\Lambda \mathrm{CDM}$ and strongly inconsistent with the GC results.

(A color version of this figure is available in the online journal.)

using Leibniz's rule to find

$$
\begin{aligned}
v_{\mathrm{c}}^{2}(r) & =-\frac{r}{v} \frac{d\left(v \sigma_{r}^{2}\right)}{d r} \\
& =\frac{r^{2}}{\pi v} \int_{r}^{\infty} \frac{d R_{\mathrm{p}}}{\sqrt{R_{\mathrm{p}}^{2}-r^{2}}} \frac{d}{d R_{\mathrm{p}}}\left[\frac{1}{R_{\mathrm{p}}} \frac{d\left(\Sigma v_{\mathrm{rms}}^{2}\right)}{d R_{\mathrm{p}}}\right],
\end{aligned}
$$

where $\Sigma\left(R_{\mathrm{p}}\right)$ and $v(r)$ are the projected and deprojected density profiles of the GC system, as described in Section 4.1.1. Now parameterizing the observed $v_{\mathrm{rms}}\left(R_{\mathrm{p}}\right)$ by Equation (2) with $\left(v_{\mathrm{rms}, 0}=225 \mathrm{~km} \mathrm{~s}^{-1}, R_{0}=4.24, \gamma_{\mathrm{p}}=-0.07\right.$; see Figure 12), we find a steeply rising mass profile, shown as model II in Figure 15. This model is similar to but steeper than the isotropic fiducial $\Lambda$ CDM-based model, which does not reproduce the observed increasing behavior of $v_{\mathrm{rms}}\left(R_{\mathrm{p}}\right)$. Also shown in Figure 15 is the mass profile if the central dispersion points were not excluded $\left(v_{\mathrm{rms}, 0}=234 \mathrm{~km} \mathrm{~s}^{-1}, R_{0}=3.78\right.$, $\left.\gamma_{\mathrm{p}}=0.24\right)$ : the central mass would be significantly higher, with a density cusp inside $\sim 5 \mathrm{kpc}$.

Given that the fiducial anisotropy profile does not seem to be correct, and isotropy is an arbitrary assumption, we next investigate what the data themselves can tell us about the anisotropy. This transpires in two ways: by considering the metal-poor and metal-rich GC populations as independent dynamical subsystems that must yield consistent results for the mass profile; and by making use of the observed kurtosis to directly constrain the anisotropy.

We begin by considering the system to be scale free, i.e., $v(r)$ and $\sigma_{r}^{2}(r)$ vary with radius as simple power laws with exponents $-\alpha$ and $-\gamma$, respectively, while $\beta$ is constant with radius. Then we can connect the circular velocity and projected dispersion by a constant relation

$$
v_{\mathrm{c}}\left(r=R_{\mathrm{p}}\right)=k \sigma_{\mathrm{p}}\left(R_{\mathrm{p}}\right),
$$

where $k$ is a complicated function of $\alpha, \beta$, and $\gamma$ (Dekel et al. 2005). We select a fixed intermediate radius $r=R_{\mathrm{p}}=4 ! 24$, where $\alpha_{\mathrm{MP}} \quad 2.7, \alpha_{\mathrm{MR}} \quad 3.4, \gamma_{\mathrm{MP}} \simeq-0.4$, and $\gamma_{\mathrm{MR}} \quad+0.4$. Now using either the metal-poor or metal-rich GCs for the righthand side of Equation (8) must yield the same answer for the left-hand side, so we search for combinations of $\left(\beta_{\mathrm{MP}}, \beta_{\mathrm{MR}}\right)$ that satisfy the equation $k_{\mathrm{MP}} / k_{\mathrm{MR}}=\sigma_{\mathrm{p}, \mathrm{MR}} / \sigma_{\mathrm{p}, \mathrm{MP}}$. We find that a fully isotropic solution $\left(\beta_{\mathrm{MP}}=\beta_{\mathrm{MR}}=0\right)$ is not preferred, because the steeper density and dispersion profiles of the metalrich GCs should depress $\sigma_{\mathrm{p}, \mathrm{MR}}$ relative to $\sigma_{\mathrm{p}, \mathrm{MP}}$ even more than is observed. In fact, strong tangential anisotropy $(\beta \lesssim-1)$ is implied for both the metal-poor and metal-rich GCs. The degeneracies and uncertainties among the parameters do not allow us to determine more than this (if we assume $\beta_{\mathrm{MP}}=\beta_{\mathrm{MR}}$ for simplicity, then these formally take the value of -3.6).

We now turn to constraints from the kurtosis, which as higher order LOSVD moments can provide direct information about the orbital types. It is beyond the scope of this paper to explore this theme in detail, but we can take advantage of one simplification discussed in Napolitano et al. (2009, Equation (B10)). If $\gamma=0, \beta$ is a constant, and a simplified distribution function is adopted, then $\beta$ can be directly estimated by deprojecting $\kappa_{\mathrm{p}}$ and $\Sigma_{\mathrm{p}}$-with no dynamical modeling necessary. We therefore take this approach for the overall GCS of NGC 1407, since $\gamma_{\mathrm{p}}=-0.07 \pm 0.26$. With $\kappa_{\mathrm{p}}=-0.30 \pm 0.41$ (Section 3.4), we find that $\beta=-0.5_{-1.3}^{+0.6}$, where the uncertainty is based only on the observed uncertainty in $\kappa_{\mathrm{p}}$ and does not include the 


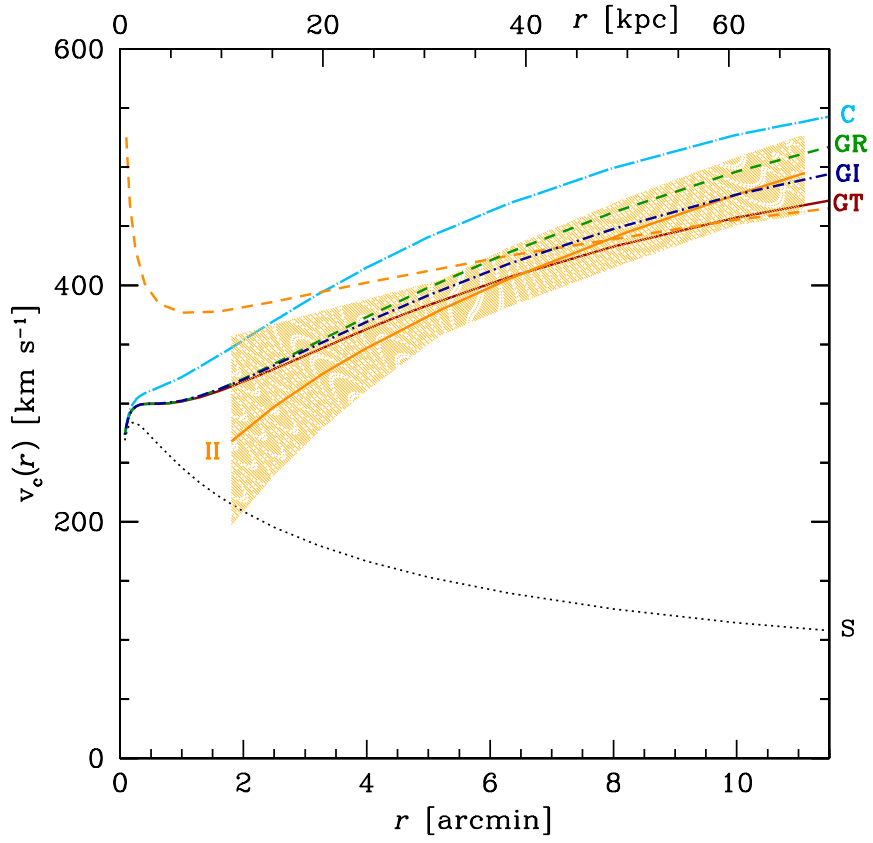

Figure 15. Mass modeling results for NGC 1407 from GCS dynamics, expressed as circular velocity profiles. Individual models are labeled by letters and are described in the text and in Table 3. The orange shaded region around model II (direct mass inversion) reflects the $68 \%$ statistical uncertainties on the dispersion profile. The orange dashed line shows model II where the dispersion data at all radii have been included.

(A color version of this figure is available in the online journal.)

uncertainties in $\Sigma_{\mathrm{p}}$ nor in the deviations of the galaxy from the simplified $(\gamma, \beta)$ model. Thus, we find an independent indication that the GC system is tangentially anisotropic overall.

Given the two separate indicators of tangential anisotropy, we now fit a $\Lambda$ CDM model to the GC data with $\beta=-0.5$ assumed. The best-fit model ("GT") provides a slightly better fit than the isotropic model (see Figure 12 and Table 3), and we therefore have three different lines of evidence for tangential anisotropy-none of which is statistically significant on its own, but in combination are suggestive of a reliable result. This solution implies a slightly lower virial mass of $\sim 6 \times 10^{13} M_{\odot}$ (Figures 14 and 15) than the isotropic model.

We can next take this best-guess model as a starting point to estimate the anisotropy profiles of the metal-poor and metal-rich subsystems. Using the method of Tonry (1983, Appendix), we take the model $v_{\mathrm{c}}(r)$ and the observed $\sigma_{\mathrm{p}}\left(R_{\mathrm{p}}\right)$ (parameterized in our case by a power-law fit), then invert them to an intrinsic $\sigma_{r}(r)$, and finally solve the Jeans equation to find $\beta(r)$ (see also Hobbs \& Willmore 1997).

This inverse approach can be sensitive to assumptions about $v_{c}$ and $\sigma_{\mathrm{p}}$ outside the regions constrained by the data, so we use it as a starting guess for simplified $\beta(r)$ functions, re-solving the Jeans equations and comparing the model $\sigma_{\mathrm{p}}$ to the data. We adopt the simplest assumption of constant anisotropy, and find that $\beta_{\mathrm{MP}} \sim-4$ (tangentially biased orbits) provides a good description for the metal-poor GCs (see Figure 16). The metal-rich GCs on the other hand appear to be roughly isotropic $\left(\beta_{\mathrm{MR}} \sim 0\right)$ outside $\sim 2^{\prime}$. The high metal-rich velocity dispersion observed at smaller radii suggests that the orbits become very radial in this region-although even $\beta_{\mathrm{MR}}=+1$ is not quite enough to reproduce the data, and to compensate, very tangential orbits would be needed in a transition region at $\sim 1.5$. These peculiarities could be caused by an admixture of DGTOs

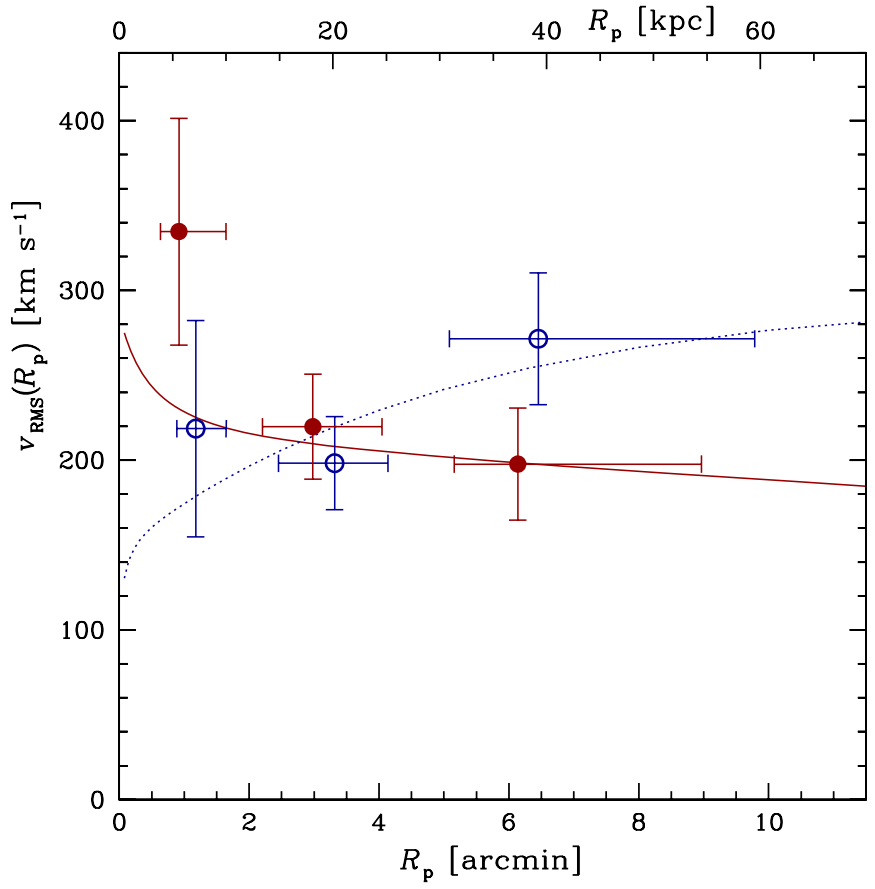

Figure 16. Projected rms velocities of NGC 1407 GCs, where curves show model predictions and points with error bars show the data. The blue dotted curve and open points show the metal-poor GCs, while the red solid curve and filled points show the metal-rich GCs. The model is the best-fit overall GC solution ("GT"), with $\beta_{\mathrm{MP}}=-4$ and $\beta_{\mathrm{MR}}=0$.

(A color version of this figure is available in the online journal.)

concentrated in the central regions as discussed in Section 3.3, and will require more detailed modeling in the future. Note that changing our assumed distance and $\Upsilon_{*}$ to higher values would have little impact on the inferred values for $\beta, M_{\mathrm{vir}}$, and $c_{\mathrm{vir}}$.

In summary, we can conclude that the GC kinematical information is compatible with a standard $\Lambda$ CDM halo with size and mass parameters similar to a very high mass galaxy group. The GCs appear to reside on somewhat tangential orbits overall, which may be decomposed into very tangential orbits for the metal-poor subpopulation and isotropic orbits for the metal-rich GCs. The anisotropy results are not yet robust, and in Section 4.3 we will explore additional constraints that may clarify the mass profile of the NGC 1407 system.

For now, assuming that this anisotropy result is correct, we report a range for the mass inside the GCS-constrained region of $10^{\prime}(60 \mathrm{kpc})$ to be $(2.7-3.2) \times 10^{12} M_{\odot}$, implying a massto-light ratio relative to the galaxy NGC 1407 of $\Upsilon_{I}=(29-34)$ $\Upsilon_{\odot, I}$, or $\Upsilon_{B}=(61-71) \Upsilon_{\odot, B}$. The systematic uncertainty from plausible ranges on the anisotropy is $\sim 20 \%$; the uncertainties from the GCS density profile and the distance are at the level of $\sim 10 \%$ and $\sim 20 \%$, respectively. The GCs with the lowest binding energies have apocenters of $\lesssim 15^{\prime} \sim 90 \mathrm{kpc}$, which does not necessarily mean that the GCS ends beyond this radius (compare Figure 11), but rather is consistent with our model of the GCs on near-circular orbits.

\subsection{X-Ray Analysis}

We next consider a fully independent constraint on the mass profile of the NGC 1407 group, making use of X-ray emission from hot gas trapped in its potential well. As a first approximation to estimating the group mass in this way, we take the ROSAT-derived X-ray temperature of $1.02 \mathrm{keV}$ (OP04), and 
use the mass-temperature relation of Vikhlinin et al. (2006) to estimate $M_{500}=3.3 \times 10^{13} M_{\odot}$ (i.e., for an overdensity of $\Delta=500)$. An alternative relation from Sanderson et al. (2006) that may be more valid in the group regime yields $M_{500}=1.7 \times 10^{13} M_{\odot}$. With reasonable NFW extrapolations to the virial radius, we thus infer $M_{\text {vir }} \sim(2-6) \times 10^{13} M_{\odot}$.

A firmer result on the mass profile requires a careful analysis based on high-resolution X-ray data. NGC 1407 was observed by Chandra (Weisskopf et al. 2002) on 2000 August 16 for $\sim 49 \mathrm{ks}$ (Obsid 791) using the ACIS instrument with the S3 chip at the focus. A new level 2 events file was produced from the level 1 events file using version 3.4 of the Chandra Interactive Analysis of Observations ${ }^{13}$ (CIAO), with CALDB version 3.3.0. Bad pixels, and events with ASCA grades of 1, 5, and 7, were removed from the analysis, and the appropriate gain map and time-dependent gain correction were applied. We eliminated flares by extracting light curves from CCDs 5 and 7, and one from the front-illuminated CCDs (2, 3, 6 and 8), and filtered these light curves using the Markevitch script lc_clean. The net remaining exposure time was $30.4 \mathrm{ks}$. Background subtraction was performed using the appropriate blank-sky data set, ${ }^{14}$ which was rescaled using the ratio of count rates at particle-dominated energies. We used the CIAO tool wavdetect to identify point sources in the data, and spectra and response files were extracted from the cleaned data using the CIAO analysis threads.

The X-ray data analysis techniques are described in detail in $\mathrm{J}+09$; a summary of the essential features is given here. Our aim is to produce a high-resolution mass profile, which is achieved through a two-stage analysis, by firstly analyzing temperature on a coarse grid, and then computing gas density on a fine grid. We extract a series of spectra from coarsely spaced concentric annuli, whose spacings are determined by a requirement of 1500 net counts in each annulus. We find that this criterion is sufficient to robustly constrain the temperature and to perform a successful deprojection of the spectra. We fit absorbed APEC models to the spectra from each coarsely spaced annulus using XSPEC version 11.3.2t, ignoring energies below $0.5 \mathrm{keV}$ and above $7.0 \mathrm{keV}$. We allow for the presence of unresolved point sources in the emission by including a power-law component of fixed index $=$ 1.56 (Irwin et al. 2003) following a de Vaucouleurs $R^{1 / 4}$ density law with $R_{\text {eff }}=1$ 1'17 (Z+07). We use the PROJCT model in XSPEC under the assumption of spherical symmetry to deproject the spectra, fitting a single global abundance value across all radial bins for the sake of deprojection stability. This yields three-dimensional model parameters at a series of characteristic coarse radii. We fit smoothing spline models to the profiles using the SMOOTH.SPLINE algorithm from the R PROJECT statistical package. ${ }^{15}$ This allows the interpolation of the fitted parameters at a series of characteristic fine radii, as described below.

It is worth noting that the temperature profile of NGC 1407 shows typical cool-core behavior, and we would expect an associated abundance gradient (Rasmussen \& Ponman 2007). In our current modeling, we fit the abundance at a constant level, which affects the APEC model normalization and thus the gas density, owing to the dominance of line emission at low temperatures. The primary effect of allowing for a gradient might be to decrease the inferred mass at the level of $\sim 20 \%$ (J+09).

The resulting temperature profile is shown in Figure 17 (left), where the vertical error bars are recovered from 100 Monte

\footnotetext{
13 http://cxc.harvard.edu/ciao/

14 http://cxc.harvard.edu/contrib/maxim/bg/

15 http://www.R-project.org/ (R Development Core Team 2006).
}

Carlo realizations of the procedure, and are used to weight the smoothing spline fit (by the inverse square of the temperature uncertainty). The most notable feature is a sharp peak of $\sim 1.6 \mathrm{keV}$ in the binned profile between $2^{\prime}$ and $3^{\prime}$. Although this feature appears significant, it may be an artifact of some instability in the deprojection procedure; comparable analyses by $\mathrm{H}+06 \mathrm{~b}$ and by $\mathrm{Z}+07$ found a peak of only $\sim 1.3 \mathrm{keV}$. The large error bar means that this point is down-weighted in the fit, but in any case, our Monte Carlo simulations do take large $T_{\mathrm{X}}$ excursions into account in the reported mass profile uncertainties.

We next extract a series of spectra from finely spaced concentric annuli, and again fit absorbed APEC models in each annulus. We use the PROJCT model in XSPEC to yield the deprojected model parameters. However, in this stage we only fit for the APEC model normalization, which directly yields the gas density. The temperature, abundance, and Galactic column in each shell are fixed at the interpolated value from the smoothing spline fits in the coarse stage. This process allows us to measure the gas density on a finer radial scale.

To determine the mass profile of NGC 1407, we use the smoothing spline function fitted to the coarse three-dimensional temperature profile, and fit a cusped "beta model" (e.g., H+06b) to the fine three-dimensional gas density profile ( $r_{\text {core }}=33^{\prime \prime}$. 0 , $\beta_{\mathrm{X}}=0.5$; see Figure 17, right). This allows the determination of the circular velocity profile under the assumption of hydrostatic equilibrium, using the equation

$$
v_{\mathrm{c}}^{2}(r)=-\frac{k_{\mathrm{B}} T_{\mathrm{X}}(r)}{\mu m_{p}}\left(\frac{d \ln n_{\mathrm{g}}}{d \ln r}+\frac{d \ln T_{\mathrm{X}}}{d \ln r}\right),
$$

where $T_{\mathrm{X}}(r)$ and $n_{\mathrm{g}}(r)$ are the gas temperature and density profiles (Fabricant et al. 1980).

We use a Monte Carlo procedure to produce 100 realizations of the mass profile of NGC 1407, which are each analyzed as described above. The span of results from these random realizations is used to define the confidence regions shown in Figure 18, where we now adopt a logarithmic radius scale in order to see the models more clearly. We find the $v_{\mathrm{c}}(r)$ profile to be consistent with constancy inside $4 \mathrm{kpc}$, and outside of this radius, it rapidly rises until $20 \mathrm{kpc}$-increasing the enclosed mass by a factor of 10-15 despite a radius increase of only a factor of 5-whereafter it may level off again. It is the rapid increase of $T_{\mathrm{X}}$ with radius (Figure 17) that produces the $v_{\mathrm{c}}$ increase; reinstating the highest $T_{\mathrm{X}}$ point in the modeling would produce an even steeper (and possibly unphysical) $v_{\mathrm{c}}$ profile. We will further discuss the features of the mass profile in the next section.

\subsection{Comparing Independent Results}

Having derived mass profiles from both GC and X-ray data, we now explore the systematic uncertainties by making independent modeling comparisons. In Section 4.3.1, we compare our own X-ray result for NGC 1407 to literature X-ray studies of this system, and in Section 4.3.2, we consider three different mass probes in NGC 1407. In Section 4.3.3, we survey similar multiple mass probes in other systems.

\subsubsection{Multiple X-Ray Studies in NGC 1407}

Several independent analyses of the same Chandra data for NGC 1407 have been published, whose results we also plot in Figure 18 for reference. Fukazawa et al. (2006) performed both a nonparametric deprojection analysis, and a parameterized "double-beta model" fit, to produce their mass profiles. $\mathrm{H}+06 \mathrm{~b}$ 

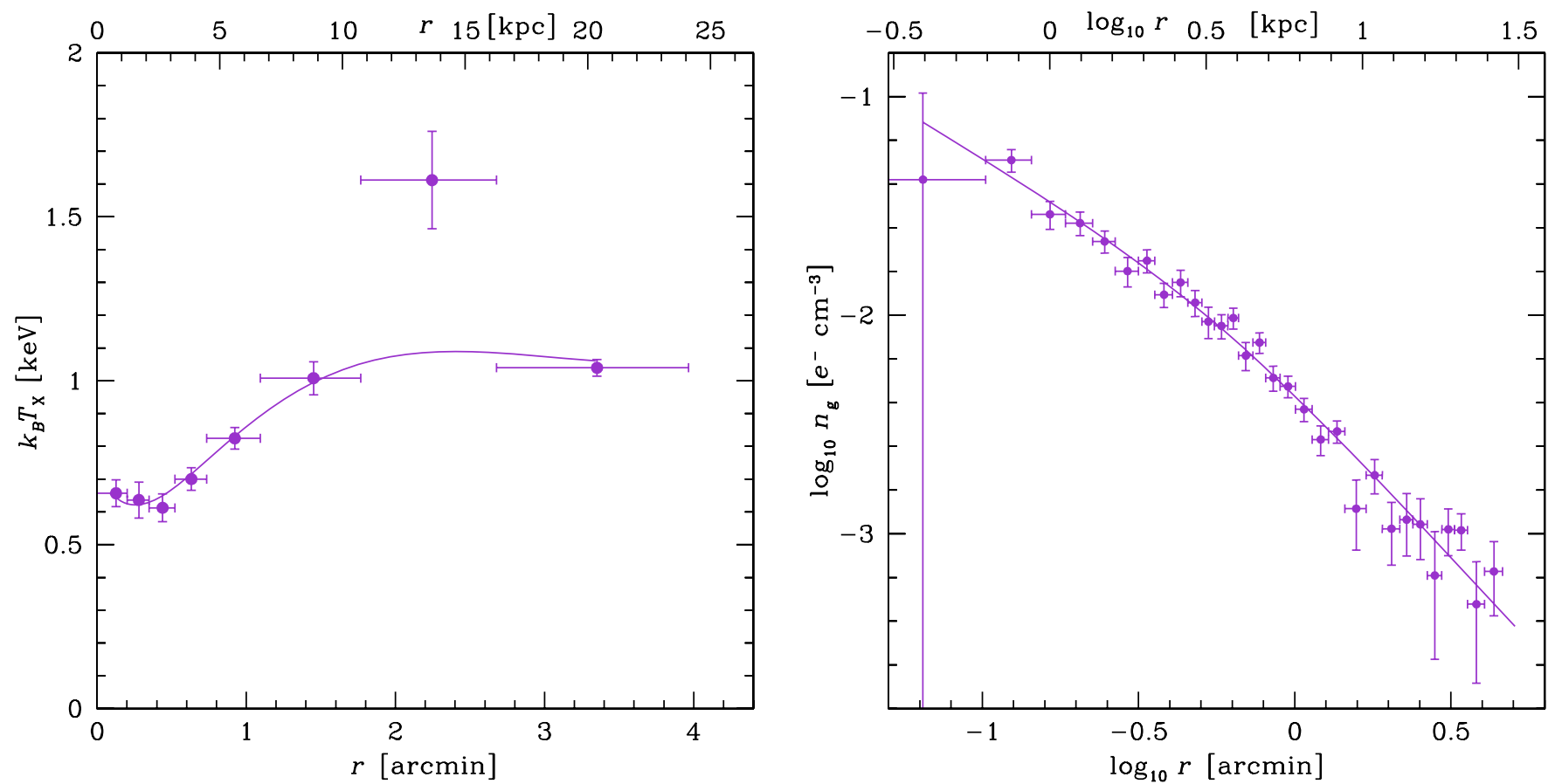

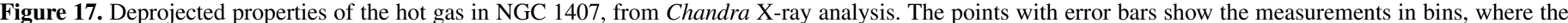

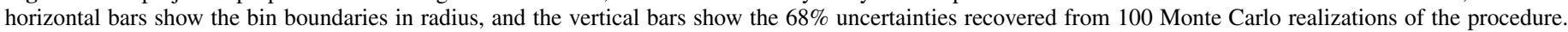

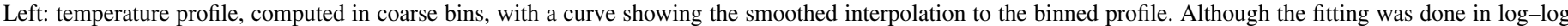

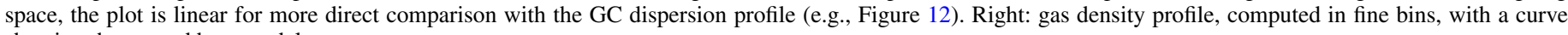
showing the cusped beta model.

(A color version of this figure is available in the online journal.)

also made deprojected models as well as forward fitting of $\Lambda \mathrm{CDM}$-based galaxy+halo models; their results extend to larger radii than ours by analyzing all of the available ACIS chips, while we conservatively used the back-illuminated S3 chip only. $\mathrm{Z}+07$ again used the Chandra data, combining them also with ROSAT data at larger radii, using both projected and deprojected double-beta models.

As seen in Figure 18, these various analyses yield qualitatively similar results for the mass profile, finding a rapid rise in $v_{\mathrm{c}}(r)$ followed by a probable leveling-off. But in quantitative detail, there are differences in the results that are larger than the claimed uncertainties. The Z+07 results agree well with ours outside 1', but inside that radius have an extra "hump" in $v_{\mathrm{c}}$ that is not seen in any other analysis. The hump is produced by their doublebeta model for the gas density, a parameterization for which we see no motivation from our higher resolution density profile. The $\mathrm{H}+06 \mathrm{~b} v_{\mathrm{c}}(r)$ deprojected result has a similar shape and amplitude to ours, but is oddly offset by a factor of $\sim 2$ in radius-perhaps owing to the different abundance treatments. We will examine these differences in more detail in another paper, and here note that there can be substantial systematic effects in deriving mass profiles from X-ray data, depending on the parameterizations and assumptions in the modeling.

Since the rapid $v_{\mathrm{c}}(r)$ rise between 5 and $20 \mathrm{kpc}$ seems to be a robust conclusion from the X-ray models, we next consider the implications of this feature. Similar "kinks" have been found in the X-ray-based mass profiles of many other galaxy groups and clusters (see Z+07 for a summary), suggesting a transition between a DM halo belonging to the central galaxy and a largerscale halo pertaining to the entire group or cluster. Such a galaxy-group halo transition would pose a problem to CDM theory, which predicts that halos should relax into smooth mass profiles similar to the NFW profile; any kink would be unstable and transient. Indeed, $\mathrm{H}+06 \mathrm{~b}$ did not find a good fit for their $\Lambda \mathrm{CDM}$-based models, which as seen in Figure 18 is probably due to an inability of such models to reproduce the kink. The model solutions that come closest are forced to higher DM halo concentrations than expected in $\Lambda \mathrm{CDM}(\mathrm{H}+06 \mathrm{~b}$ Figure 4).

We next try to fit our own galaxy+halo model sequence, using our own X-ray data analysis. In contrast to the forward-modeling performed by $\mathrm{H}+06 \mathrm{~b}$, we use a less direct method of fitting the model $v_{\mathrm{c}}(r)$ to our deprojected X-ray profile. The fit is performed at 31 points in radius, but since there were effectively seven independent data points in the original coarse-binned data, we renormalize the $\chi^{2}$ statistic by a factor of $7 / 31$. Our best-fit solution (model XR; see Table 3 ) is shown in Figure 18. Again, the NFW-based model has difficulty reproducing the kink and requires a DM halo that is more concentrated than is typical for $\Lambda C D M$ (see Figure 14).

To see if we can find a better solution, we re-examine our modeling assumptions. The distance to the galaxy, and $\Upsilon_{*}$, are both key parameters that could plausibly have higher values (see Section 4.1.1). However, increasing either of them causes the $\mathrm{X}$-ray fit to deteriorate rapidly (driven by the constraints in the central regions). Decreasing either of these parameters (e.g., to $\Upsilon_{*, I}=1.52 \Upsilon_{\odot, I}$ ) yields better fits, but also pushes the halo concentration to higher values.

As a single case, the high concentration of NGC 1407 is not a problem, but we note that most of the $\mathrm{X}$-ray bright galaxies and groups examined by H+06b and by Gastaldello et al. (2007) were also found to have high concentrations, and unconventionally low values for $\Upsilon_{*}$ (see also the high concentrations in Schmidt \& Allen 2007; Duffy et al. 2008; Broadhurst et al. 2008; Broadhurst \& Barkana 2008). It has been shown that improper modeling 


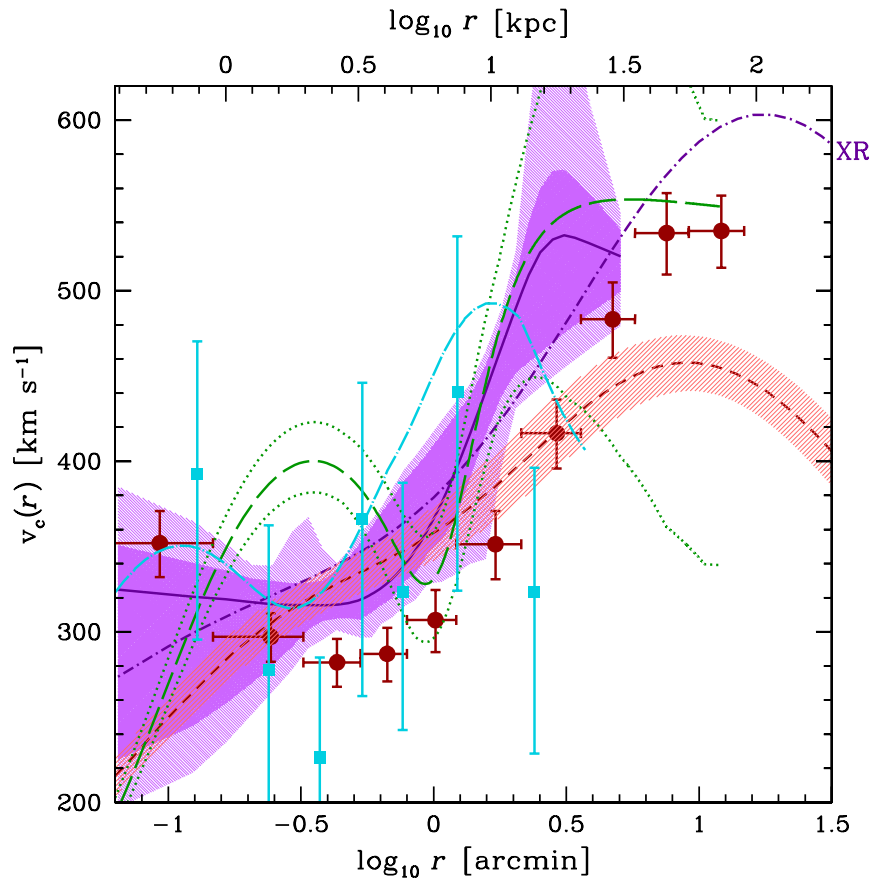

Figure 18. X-ray-based mass modeling results for NGC 1407. Our new Chandra-based result is shown as a purple solid curve with shaded regions showing the $1 \sigma$ and $2 \sigma$ uncertainty regions. The best-fit stars+NFW solution (model XR) is shown as a purple dot-short-dashed curve. The Chandra-based results of Fukazawa et al. (2006) are shown, including their double-beta model fit (blue dot-long-dashed curve) and their deprojection analysis (blue boxes with error bars). The Chandra-based results of $\mathrm{H}+06 \mathrm{~b}$ include their $\Lambda \mathrm{CDM}$-based model fit without adiabatic contraction (red short dashed curve with shaded uncertainty region) and their deprojection analysis (red circles with error bars). The Chandra+ROSAT analysis of $\mathrm{Z}+07$ is shown by the green long-dashed curve, with green dotted curves showing the $90 \%$ uncertainties.

(A color version of this figure is available in the online journal.)

of the stellar mass in galaxies can strongly skew X-ray-based inferences about their DM halos (Mamon \& Łokas 2005a). However, in the cases of NGC 1407 as well as the other galaxies studied by $\mathrm{H}+06 \mathrm{~b}$ (see also $\mathrm{H}+08$ ), it seems that the $\mathrm{X}$-ray data simply do not allow for plausible $\Upsilon_{*}$ values. $\mathrm{H}+06 \mathrm{~b}$ found best fits for NGC 1407 corresponding to $\Upsilon_{*, B} \sim 2-3 \Upsilon_{\odot, B}$, as compared to their stellar population estimate of $\sim 5-6 \Upsilon_{\odot, B}$ and ours of $\Upsilon_{*, B} \sim 4-7 \Upsilon_{\odot, B}$ (see Section 4.1.1; using our shorter adopted distance would lessen this discrepancy).

We illustrate the situation further in Figure 19 using the deprojected X-ray results of $\mathrm{H}+06 \mathrm{~b}$ for their entire sample (revising the distance to $21 \mathrm{Mpc}$ for NGC 1407). Presented as circular velocity profiles, several trends are immediately apparent. Three galaxies (NGC 720, NGC 4125, and NGC 6482) have flat or declining profiles consistent with galaxy-scale halos (modulo some issues with the innermost data points). The other four galaxies have increasing profiles suggesting group-scale halos (compare also Nagino \& Matsushita 2009). However, these increases generally set in much too quickly (by $\sim 1 R_{\text {eff }}$ ), as can be seen by comparison with the $\Lambda$ CDM-based model for NGC 1407. In order to reproduce these kinks, a combination of low $\Upsilon_{*}$ and high $c_{\text {vir }}$ is necessary (though still not sufficient).

The combination of awkward mass profile kinks, and nonstandard stellar and DM mass parameters, leads us to suspect a systematic problem with some X-ray mass inferences. The kinks found by $\mathrm{H}+06 \mathrm{~b}$ seem to coincide with strong increases in $T_{\mathrm{X}}$, which are examples of the common "cool core" phenomenon found in galaxy groups and clusters (e.g., Sun et al. 2009). Inde-

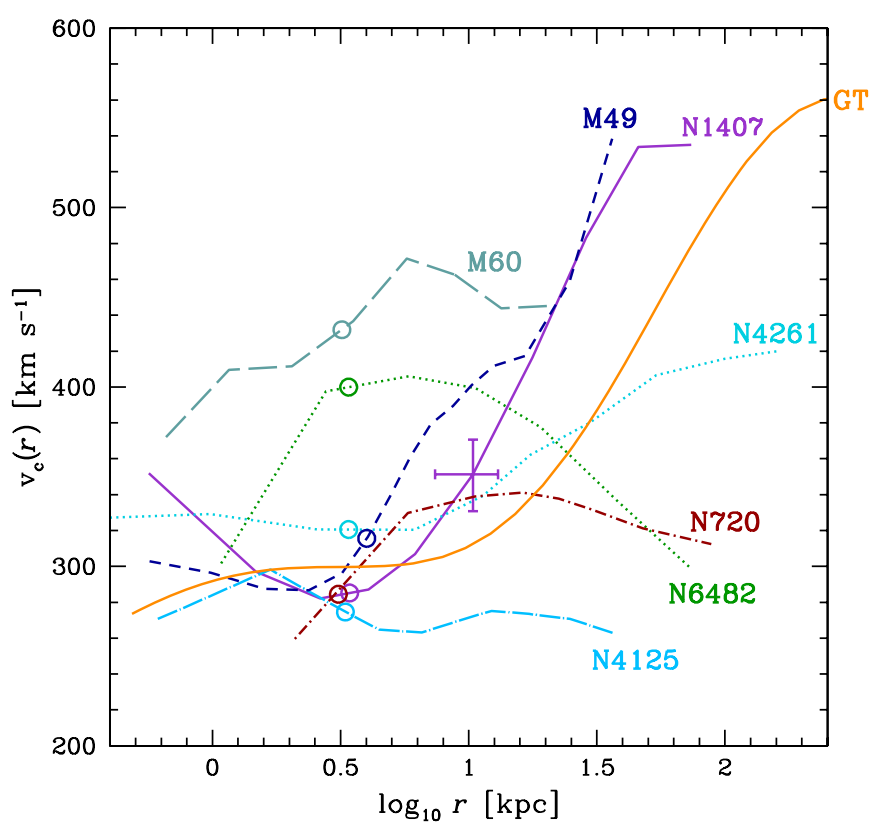

Figure 19. Circular velocity profiles of seven $\mathrm{X}$-ray bright galaxies from $\mathrm{H}+06 \mathrm{~b}$. Also shown is our best-fit GC-based solution for NGC 1407 (solid orange curve; model GT). The open circles mark $1 R_{\text {eff }}$ (in the $K_{s}$-band) for each galaxy. Error bars show the typical radial binning and mass uncertainty at one measurement point.

(A color version of this figure is available in the online journal.)

pendently of the wider implications of the cool cores, we wish to know whether there is a problem with applying Equation (9) in these regions. In the case of NGC 1407, the onset of the kink coincides not only with a rapid $T_{\mathrm{X}}$ increase, but also with an onset of increasing gas isophote ellipticity (from $e=0.0$ at 0.6 to $e=0.3$ at $1^{\prime} .7$ ) and slight asymmetry. It thus seems plausible that the gas in the kink region is not in hydrostatic equilibrium, or that there is additional nonthermal pressure support inside the kink radius (which might produce mass underestimates in these regions). Testing these possibilities with independent mass tracers is exactly one of the motivations of the present GCS kinematics study, as we will see in the following sections.

\subsubsection{Multiple Mass Probes in NGC 1407}

We next compare results from the available independent probes on the mass profile of the NGC 1407 group. These constraints include the GC and X-ray analyses that we presented in Sections 4.1 and 4.2, as well as the dynamics of galaxies in the group $(B+06 b)$, and are summarized in Figures 14 and 20.

$\mathrm{B}+06 \mathrm{~b}$ used a friends-of-friends algorithm to identify 19 galaxies belonging to the group, and a virial estimator to convert the kinematics into a mass. Their galaxy sample notably excluded NGC 1400, the bright early-type apparently in close three-dimensional proximity to NGC 1407 , but with a high peculiar velocity. $\mathrm{B}+06 \mathrm{~b}$ made an alternative mass estimate including NGC 1400 and another five galaxies in the region of the group-yielding both a higher group mass and a higher group luminosity; $\mathrm{B}+06 \mathrm{a}$ provided yet another updated mass that is intermediate to the previous two. The results for both the low- and high-mass group samples are shown in Figure 20, implying a mass at $0.5 \mathrm{Mpc}$ of $(4-10) \times 10^{13} M_{\odot}$, or $\Upsilon_{B}=$ $600-1200 \Upsilon_{\odot, B}$ (the corresponding group velocity dispersion is $\sigma_{\mathrm{p}}=320-450 \mathrm{~km} \mathrm{~s}^{-1}$ ). Trentham et al. (2006) identified 10 new dwarf galaxies belonging to the group, and estimated a virial mass of $6.1 \times 10^{13} M_{\odot}$. We plan to revisit this calculation 


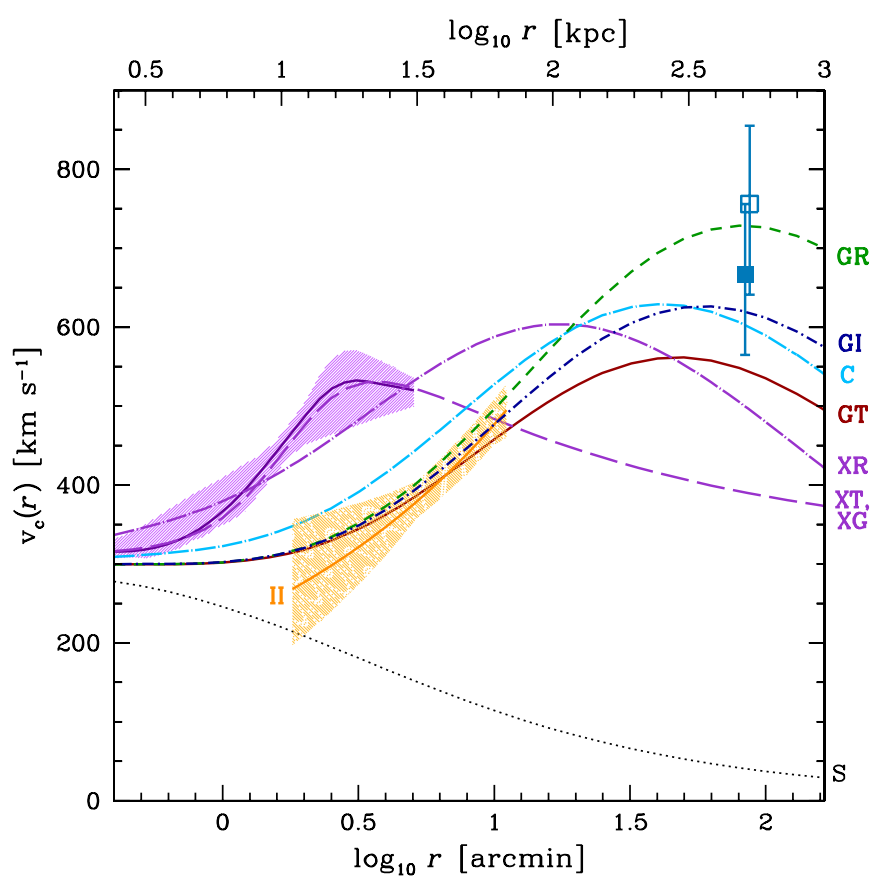

Figure 20. Circular velocity profile of NGC 1407, plotted vs. log radius Individual models are labeled by letters and are described in the text and in Table 3. Further included is our X-ray result (Section 4.2). The boxes with error bars show estimates from group galaxy dynamics $(B+06 b)$, using the friendsof-friends sample (filled box) or the entire sample of galaxies in the projected region (open box)

(A color version of this figure is available in the online journal.)

in the future with the addition of even more group dwarfs to the catalog. Using the $\mathrm{B}+06 \mathrm{~b}$ low-mass results for now, the permitted regions of $M_{\mathrm{vir}}-c_{\mathrm{vir}}$ parameter space are shown in Figure 14.

The orbit anisotropy of the group galaxies is an additional source of systematic uncertainty as with the GC dynamics; the virial mass estimator used by $\mathrm{B}+06 \mathrm{~b}$ implicitly assumes isotropy. Somewhat conflicting conclusions have been made about the accuracy of isotropic mass estimators in galaxy groups and clusters in general (Sommer-Larsen 2006; Biviano et al. 2006; Benatov et al. 2006; Faltenbacher \& Mathews 2007; Hwang \& Lee 2008). In the case of the NGC 1407 group, B+06b found a near-zero skewness for the galaxy velocities as well as a crossing time of only $0.02 \mathrm{H}_{0}^{-1}$, supporting the assumption of the group as virialized, and some indication of negative kurtosis, suggesting that isotropic estimators might overestimate the mass. In any case, the systematic mass uncertainty is probably no larger than the $\pm 40 \%$ statistical uncertainty.

Now comparing the results from the three mass probes, we see that they each independently imply a virial mass for the NGC 1407 group within the range $M_{\text {vir }} \sim(4-10) \times 10^{13} M_{\odot}$ (Figure 14). This is an encouraging broad consistency, but we are further interested in the accuracy of the spatially resolved mass profiles. The group galaxies and GC dynamics results are consistent (assuming fiducial DM models and any of the GC anisotropy profiles discussed in Sections 4.1.3 and 4.1.4), providing no evidence so far of a major problem with either approach. But snags appear when considering the X-ray mass profile: while the virial mass from the group galaxies and the $\mathrm{X}$ rays is compatible (Figure 14), the mass profile at smaller radii conflicts both with the theoretical expectations for a $\Lambda \mathrm{CDM}$ halo (see also Section 4.3.1) and with the GC-derived profile.
The best-fitting CDM halo parameters from the GC and X-ray data are inconsistent at the $2 \sigma$ level. This discrepancy also exists independently of the mass model assumed, as seen in the overlap region $\left(\sim 2.5-5^{\prime}\right)$ of Figure 20, where the unparameterized mass profile inferred from the GCs is $\sim 70 \%$ lower than the $\mathrm{X}$-ray result. This difference is approximately halved when considering instead the deprojected X-ray profile of $\mathrm{H}+06 \mathrm{~b}$, but the problem persists.

Our GC results are not yet robust, with the orbital anisotropy as the key remaining source of potential error. To see if this systematic uncertainty could account for the GCS/X-ray discrepancy, we construct a toy model where the X-ray-based mass profile is assumed to be correct, and investigate the GCS dynamics in the corresponding potential. To represent the X-ray result, we construct a $v_{\mathrm{c}}(r)$ profile with an arbitrary functional form

$$
v_{\mathrm{c}}(r)=v_{0}+\frac{v_{1} r^{\delta}}{1+\left(r_{\mathrm{c}} / r\right)^{\zeta}} .
$$

Model XT/XG has parameters $\left(v_{0}=312 \mathrm{~km} \mathrm{~s}^{-1}, v_{1}=\right.$ $\left.404 \mathrm{~km} \mathrm{~s}^{-1}, r_{\mathrm{c}}=1.94, \zeta=3.1, \delta=-0.37\right)$ and is shown in Figure 20; the range of plausible values for the asymptotic profile exponent $\delta$ is between -0.5 and +0.08 .

Assuming $\beta=-0.5$ for the GCS as derived in Section 4.1.4, we carry out a Jeans analysis in this mass potential, and illustrate the resulting prediction for $\sigma_{\mathrm{p}}\left(R_{\mathrm{p}}\right)$ in Figure 12 (model XT). This model is a poor fit to the data, mainly because the relatively high X-ray-derived $v_{\mathrm{c}}$ at $\sim 3^{\prime}$ overpredicts $\sigma_{\mathrm{p}}$ for the GCs around this intermediate radius. Setting aside our estimate for the anisotropy, we next investigate what profile $\beta(r)$ would be required to reproduce the $\mathrm{GC}$ observations, given the $\mathrm{X}$ ray mass model (compare Mathews \& Brighenti 2003). Some qualitative experimentation reveals that in order to suppress $\sigma_{\mathrm{p}}$ at intermediate radii while keeping $\sigma_{\mathrm{p}}$ high at larger radii, the GC orbits must be very radial in the center, and then rapidly become tangential at large radii. More quantitatively, we use the Tonry inversion method (see Section 4.1.4) to derive $\beta(r)$ for the GCS, given the observed $\sigma_{\mathrm{p}}\left(R_{\mathrm{p}}\right)$ (outside the central regions) and the assumed $v_{\mathrm{c}}(r)$.

The result of this mass-anisotropy inversion is shown in Figure 13: the GC orbits are near-isotropic in a narrow range around $r \sim 8^{\prime}$, becoming very tangential at larger radii and very radial at smaller radii (with an unphysical $\beta>1$ for $r \lesssim 3^{\prime}$ ). Interestingly, while the dispersion data inside 1.75 were not used in this analysis, the model does fit them a posteriori (see model XG in Figure 12, which uses a parameterized approximation to the numerically inverted anisotropy profile). This agreement lends credence to the existence of some objects (possibly DGTOs; Section 3.3) on very radial orbits near the galaxy center, but it is not clear that this scenario would solve the GC/X-ray inconsistency problem (see the dashed curve in Figure 15).

These anisotropy conclusions are not substantially affected by the extrapolation of the X-ray mass profile (parameter $\delta$ in Equation (10)), nor by the uncertainties in the GC density distribution $v(r)$; they are also completely independent of the distance assumed. Adopting the alternative X-ray-based mass profile from $\mathrm{H}+06 \mathrm{~b}$ (which notably accounts for a metallicity gradient) does not substantially change the $\beta(r)$ result. Reconciling the GC and X-ray data therefore seems to require an ad hoc GC anisotropy profile with extreme radial variations, conflicting with the anisotropy inferred from the GC data themselves. As we will discuss in Section 5.1, this profile is also highly implausible on theoretical grounds. 
We therefore have apparently found a fundamental incompatibility between the GC and X-ray results. However, it is premature at this juncture to pinpoint the culprit, since there are puzzling quirks in both analyses (the X-ray kink and the central $\mathrm{GC}$ dispersion peak). More exhaustive study of this system will be needed in the future, including stronger empirical constraints on the GC anisotropy (see Section 4.3.3) and a dynamical analysis of the field star kinematics-which should provide a crucial cross-check on the central X-ray mass profile (compare the study of M60 by $\mathrm{H}+08$, where the mass inside $1 R_{\text {eff }}$ derived from stellar dynamics may be significantly higher than the X-ray-based mass).

For now, we wish to estimate the best mass model that jointly fits all of the constraints, surmising in the spirit of the centrallimit theorem that the various systematic problems will roughly cancel out. The inconsistencies between the different constraints do suggest that we not naively use the usual method of simply adding up all of their $\chi^{2}$ values, but rather attempt to account for the systematic uncertainties through a weighting scheme. In particular, we do not wish the tighter constraints from the X-ray analysis to dominate the final solution.

Combining weighted data sets is a nontrivial exercise (see, e.g., Press 1997; Hobson et al. 2002), and for now we adopt the following ad hoc approach. We renormalize the $\chi^{2}$ corresponding to the X-ray and GC constraints by their best-fit values when each of them is fitted independently, including the $\Lambda \mathrm{CDM}$ concentration prior. The new $\chi^{2}$ functions, as well as the unmodified group-galaxies $\chi^{2}$ (corresponding to the total mass inside $500 \mathrm{kpc}$; see Figure 20), are summed along with the concentration prior to produce a new total $\chi^{2}$.

The resulting "consensus" solution is a normal $\Lambda \mathrm{CDM}$ halo with $M_{\text {vir }} \sim 7 \times 10^{13} M_{\odot}$ (see model C in Table 3 and Figures 14 and 20). With this solution, DM becomes dominant over the baryonic mass outside of $r \sim 1.3\left(\sim 1.4 R_{\text {eff }} \sim 8 \mathrm{kpc}\right)$. The benchmark mass-to-light ratio gradient parameter introduced by Napolitano et al. (2005) is $\nabla_{\ell} \Upsilon \sim 2.0$ for NGC 1407, which is by far the highest value reported for any early-type galaxy, demonstrating the extreme dominance of DM in this system. Note that the DM scale radius $r_{s}$ is much larger than the extent of the GC kinematics data $(120 \mathrm{kpc}$ vs. $60 \mathrm{kpc})$, so it is really the group galaxy kinematics that determines $M_{\mathrm{vir}}$, with $c_{\text {vir }}$ then pinned down by the GC and X-ray constraints. ${ }^{16}$ In the near future, it should be possible to push the GC kinematics measurements out to larger radii, and gain a stronger constraint on $M_{\text {vir }}$ without reference to the group galaxies.

One alternative estimate for the mass profile would be derived from the GCs and group galaxies only, disregarding the X-ray data and the $\Lambda \mathrm{CDM}$ concentration prior. The resulting model GG has a mass of $M_{\mathrm{vir}} \sim 1.1 \times 10^{14} M_{\odot}$ and a slightly low concentration (Figure 14).

\subsubsection{Multiple Mass Probes in Other Systems}

More detailed analyses of the current and future data, including direct constraints on the GCS anisotropy, should provide a more accurate picture of the NGC 1407 mass profile. But given the apparent conflicts in this case between independent mass constraints, we pause to review similar comparisons that are available in other early-type galaxies. Such an approach has become commonplace in the arena of galaxy clusters, where

\footnotetext{
16 The crossing time for the outer GCs is only $\sim 0.2 \mathrm{Gyr}$, and for the group galaxies is $\sim 0.3 \mathrm{Gyr}$, so nonequilibrium effects are unlikely to be the explanation for the high mass inferred.
}

gravitational lensing, $\mathrm{X}$-rays, and galaxy dynamics may all be used as cross-checks and to provide stronger constraints when used jointly (e.g., Girardi et al. 1998; Sand et al. 2004; Benatov et al. 2006; Mahdavi et al. 2008). But comparable studies in galaxies are in their infancy, as the data are difficult to acquire.

A handful of galaxy halos have been studied using both Xrays and kinematics; these are mostly cases of central group and cluster galaxies with GC data, but sometimes also with PN kinematics (e.g., Cohen \& Ryzhov 1997; Kronawitter et al. 2000; RK01; C+01; Napolitano et al. 2002; C+03; Richtler et al. 2004; Fukazawa et al. 2006; Bridges et al. 2006; Schuberth et al. 2006; Samurovic 2006; Wu \& Tremaine 2006; Richtler et al. 2008, hereafter R+08; Chakrabarty \& Raychaudhury 2008; $\mathrm{J}+09$ ). Within the uncertainties, most of these X-ray/kinematics intercomparisons are broadly consistent: the discrepancy we find in NGC 1407 is one of the largest seen. ${ }^{17}$ However, the kinematical tracers in these studies have generally been assumed to be fairly isotropic - which may indeed turn out to be typical for the GCs in group/cluster central galaxies, but is not yet certain.

Given the freedom to adjust orbital anisotropies, one could make almost any two data sets agree (e.g., Section 4.3.2), so to conclusively compare mass results, one must derive independent constraints on anisotropy. This is possible by measuring higher order shapes of the LOSVDs, which can be used to constrain the types of orbits (compare our crude kurtosis analysis in Section 4.1.4). Such mass/anisotropy degeneracy breaking techniques are well established for integrated-light stellar kinematics, but can also be applied to discrete velocities (e.g., RK01; Romanowsky et al. 2003; Wu \& Tremaine 2006; De Lorenzi et al. 2008; Chanamé et al. 2008). Although it is commonly supposed that a few hundred velocities are not sufficient to break this degeneracy, in fact additional constraints may be brought to bear on the problem. One is to make use of stellar kinematics including their LOSVDs to pin down the mass profile out to $\sim 1-2 R_{\text {eff }}$ (compare RK01). Another is to assume that the underlying spatial profile of the tracer population is fairly well known, e.g., the total GC surface density profile represents the same population as the kinematically measured GCs (compare Merritt \& Saha 1993; Merritt \& Tremblay 1993).

So far, the only galaxy to have its DM content studied using both high-quality X-ray and optical data (including LOSVD constraints) is M87. RK01 used nonparametric "orbit modeling" of the dynamics of the GCs and field stars in this galaxy, deriving a mass profile that may be compared with the subsequent $X M M$ Newton result of Matsushita et al. (2002). As seen in Figure 21, the optical and X-ray results in the region of overlap are nicely consistent, except at small radii (less than $2 \mathrm{kpc}$ ), where the typical problem of an X-ray core mass underestimate is apparent (e.g., Brighenti \& Mathews 1997; H+08).

Churazov et al. (2008, hereafter $\mathrm{C}+08)$ have recently used new Chandra data to examine the mass profile of M87 in more detail, and again found that the optical and X-ray results on the mass profile are broadly consistent, but that the X-ray profile contains rapid radial variations that are not seen (by construction) in the smooth optical models. These "wiggles" appear at a nuisance level when examining the gravitational potential, but when differentiated to derive a profile of $v_{\mathrm{c}}(r)$ or $M(r)$, the wiggles are amplified catastrophically, even implying at one location $(\sim 10 \mathrm{kpc})$ a negative enclosed mass (see

\footnotetext{
17 Mathews \& Brighenti (2003) found a discrepancy between X-ray and dynamics-based mass profiles in the central regions of M49.
} 


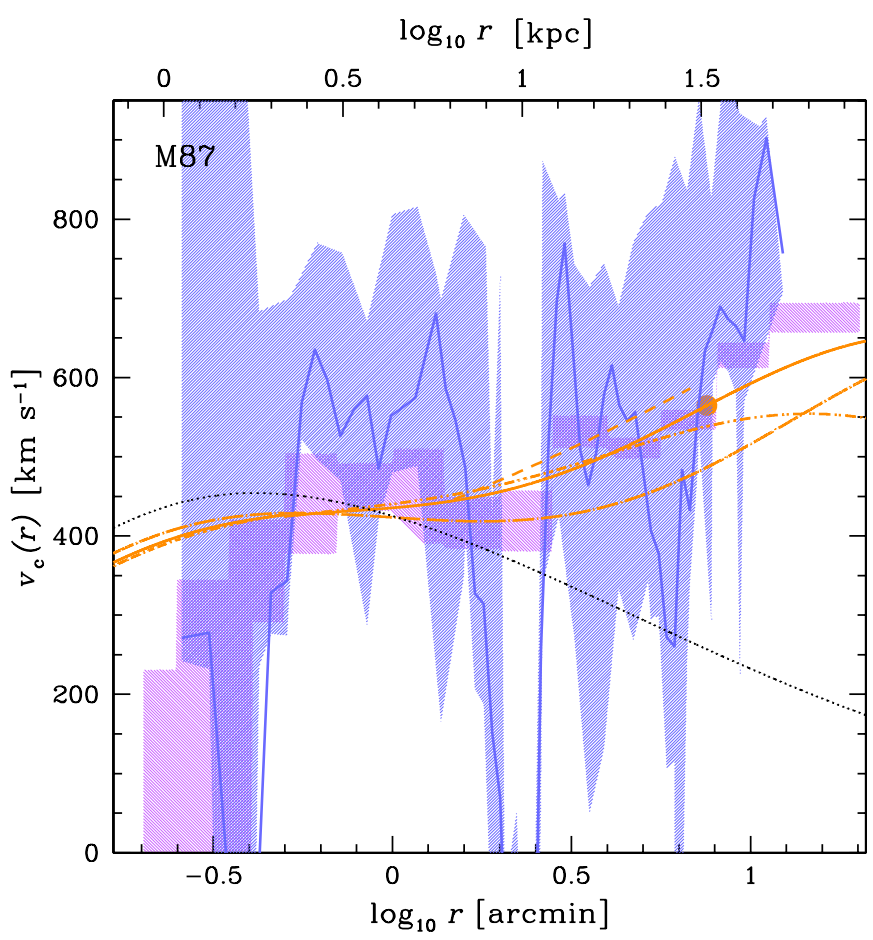

Figure 21. Mass modeling results for M87. The orange solid curve shows the best-fitted $\Lambda$ CDM-based orbit model to the stellar and GCS kinematics; the dot-dashed curves show other acceptable solutions (RK01). The filled circle shows the outer extent in radius of the kinematics data fitted. The orange dashed curve shows the result of anisotropic distribution-function-based modeling of the GCS kinematics by Wu \& Tremaine (2006), with the extent of the curve showing the radial range of the data fitted. The purple shaded region shows the XMM-Newton-based mass model of Matsushita et al. (2002). The blue jagged solid line with shaded region shows the Chandra-based model of $\mathrm{C}+08$ (mean molecular weight $\mu=0.61$ assumed), with $4 \times$ radial binning applied to smooth out the highest frequency variations; the uncertainty region incorporates the differences from single- and multitemperature modeling. The black dotted curve shows a model with no DM. The figure thus shows that the detailed optical kinematics constraints are broadly consistent with the X-ray mass results, but without artificial smoothing, the X-ray data imply an unphysical mass profile.

(A color version of this figure is available in the online journal.)

Figure 21 and Ghizzardi et al. 2004). The wiggles were not seen in the earlier XMM-Newton study because of its use of smooth analytical models to describe the gas density and temperature profiles. This standard practice avoids amplifying noise in low$\mathrm{S} / \mathrm{N}$ data, but in the case of M87, it appears to have suppressed real features in the data (see also J+09).

$\mathrm{C}+08$ showed that the apparent M87 mass wiggles could be readily explained as a consequence of shock waves propagating through the gaseous interstellar medium. They claimed that one can ignore these localized effects and still safely consider global changes in the mass potential, taking also into account additional (apparently minor) corrections to the X-ray-based mass such as nonthermal pressure support (see also Pizzolato et al. 2003; Ciotti \& Pellegrini 2004; Pellegrini \& Ciotti 2006; Rasia et al. 2006; Nagai et al. 2007; Diehl \& Statler 2007; O'Sullivan et al. 2007). However, in practice, it is difficult to know a priori how to smooth out the X-ray wiggles, and thus the conclusions derived about DM halo properties in detail can be strongly affected. NGC 1407 is a case in point, as its X-ray kink feature could be attributed to a shock, but because we do not have more extended $\mathrm{X}$-ray data to provide the asymptotic mass profile, the feature forces the $\Lambda \mathrm{CDM}$ models toward high concentrations and low values for $\Upsilon_{*}$. However, there is less a priori reason to suspect AGN-generated shocks in NGC 1407, as its X-ray image shows no major disturbances, and its radio activity is $\sim 1000$ times less than that of M87 (Condon et al. 1998).

M87 has clearly disturbed X-ray isophotes, while there may also exist a class of fairly undisturbed systems where the Xray results will be very reliable. Diehl \& Statler (2008b) have introduced a parameter $\eta$ to describe asymmetries in gaseous $\mathrm{X}$-ray halos. The correlations of $\eta$ with central radio and $\mathrm{X}$ ray luminosity, and with outer temperature gradient, suggest that both the ambient medium and the central AGN cause disturbances in the X-ray gas-even in cases such as NGC 1407 where the AGN activity appears fairly weak.

Values of $\eta$ were provided for most of the galaxies with $\mathrm{X}$ ray/kinematics mass comparisons (see references at the beginning of Section 4.3.3). The galaxies are M60, M49, NGC 1399, NGC 4636, and NGC 1407, with $\eta=0.05,0.08,0.09,0.09$, and 0.17 , respectively. NGC 1407 has a relatively high value of $\eta$ (despite having less obvious disturbances than, e.g., NGC 4636) and manifests a mass discrepancy, but there is otherwise no obvious correlation between $\eta$ and the reliability of the Xray results. So far, none of the other galaxies clearly show a large mass discrepancy, but all of them have at least a hint of a nonequilibrium kink in the mass profile-even in the case of M60, which has one of the smoothest gas halos in the Diehl \& Statler (2008b) sample.

On a related note, four of the seven galaxies studied in $\mathrm{X}$-rays by $\mathrm{H}+06 \mathrm{~b}$ have deprojected mass profiles that are inconsistent with the forward-fitted smooth NFW-based models (see Section 4.3.1). These are again cases where the X-ray approach may be problematic, but the $\eta$ values for them and the other four galaxies are not clearly different. C+08 did note that $\eta$ may not be a reliable diagnostic of hydrostatic equilibrium, and we speculate that a metric based on radial (rather than azimuthal) fluctuations in the X-ray photometry and/or temperature may be more useful for this purpose.

The generic reliability of X-ray mass constraints could be further tested by applying robust dynamical models (as in the case of M87) to the stellar and GC data in NGC 1407 and in the other galaxies mentioned (while also extending the models to consider nonspherical effects). These are demanding exercises that we defer to future papers. One might consider the X-ray analyses more suspect owing to the highly uncertain physics involved, but even in the relatively well-posed case of gravitational dynamics, there are questions about the reliability of the modeling results (e.g., Dekel et al. 2005). The latter issue can be addressed by cross-checking independent kinematical constraints, e.g., from PNe, and from the metal-poor and metalrich GC subsystems separately. We hope in the future to pursue these avenues in NGC 1407 as well as in other galaxies; studies with GCs and PNe will also be useful for testing the presumed association between metal-rich GCs and field stars.

\section{THE GROUP CONTEXT}

Having determined some properties of the mass distribution and GC kinematics in the NGC 1407 group, we now attempt to place this system in the context of what is known and expected for galaxy groups and clusters. Are the high $\Upsilon$ value and rising $v_{\mathrm{c}}(r)$ profile really unusual for groups of comparable luminosity? We may alternatively consider the system as a dim cluster, since its mass approaches the usual (arbitrary) group/ cluster boundary of $10^{14} M_{\odot}$ : is it rare for clusters to have been so inefficient in forming stars? How do the GCS kinematics compare to those of other systems? 
Table 4

Kinematical Properties of Nearby Group/Cluster-Central Galaxies

\begin{tabular}{|c|c|c|c|c|c|c|c|c|c|c|c|}
\hline NGC & Type & Env. & $N_{\mathrm{GC}}$ & $\begin{array}{c}D \\
(\mathrm{Mpc})\end{array}$ & $M_{B}$ & $\begin{array}{c}\sigma_{0} \\
\left(\mathrm{~km} \mathrm{~s}^{-1}\right)\end{array}$ & $\begin{array}{c}v_{\mathrm{rot}} / \sigma_{\mathrm{p}} \\
(\mathrm{MPGCs})\end{array}$ & $\begin{array}{c}v_{\text {rot }} / \sigma_{\mathrm{p}} \\
(\mathrm{MRGCs})\end{array}$ & $\begin{array}{c}v_{\mathrm{rms}} \\
\text { (MPGCs) }\end{array}$ & $\begin{array}{c}v_{\mathrm{rms}} \\
(\mathrm{MRGCs})\end{array}$ & Ref. \\
\hline 1399 & E1 & $\mathrm{CCG}$ & 228 & 18.5 & -21.1 & $344 \pm 6$ & $0.19_{-0.19}^{+0.11}$ & $0.11_{-0.11}^{+0.10}$ & $311_{-20}^{+24}$ & $258_{-17}^{+22}$ & $\mathrm{D}+04, \mathrm{R}+04$ \\
\hline 1407 & E0 & GCG & 172 & 20.9 & -21.0 & $273 \pm 6$ & $0.18_{-0.18}^{+0.11}$ & $0.24_{-0.18}^{+0.13}$ & $234_{-16}^{+21}$ & $247_{-17}^{+21}$ & this paper \\
\hline 3379 (M105) & E1 & GCG & 38 & 9.8 & -19.9 & $207 \pm 2$ & $\ldots$ & $\ldots$ & $\ldots$ & $\ldots$ & $B+06 c$ \\
\hline 4472 (M49) & E2 & GCG & 127 & 15.1 & -21.7 & $291 \pm 8$ & $0.30_{-0.22}^{+0.14}$ & $0.26_{-0.26}^{+0.16}$ & $373_{-29}^{+38}$ & $215_{-23}^{+28}$ & $\mathrm{C}+03$ \\
\hline 4486 (M87) & E3 & CCG & 144 & 14.9 & -21.5 & $332 \pm 5$ & $0.33_{-0.20}^{+0.13}$ & $0.66_{-0.23}^{+0.19}$ & $414_{-32}^{+39}$ & $383_{-35}^{+47}$ & $\mathrm{H}+01$ \\
\hline 4636 & E2 & GCG & 88 & 13.6 & -19.8 & $203 \pm 3$ & $0.17_{-0.17}^{+0.15}$ & $0.57_{-0.57}^{+0.36}$ & $209_{-20}^{+25}$ & $180_{-18}^{+26}$ & $S+06$ \\
\hline 4649 (M60) & E2 & GCG & 59 & 15.6 & -21.3 & $336 \pm 4$ & $0.48_{-0.28}^{+0.19}$ & $0.53_{-0.53}^{+0.26}$ & $215_{-23}^{+31}$ & $178_{-28}^{+45}$ & $\mathrm{~L}+08$ \\
\hline 5128 & $\mathrm{E} 2 / \mathrm{S} 0$ & GCG & 170 & 3.9 & -20.7 & $120 \pm 7$ & $0.54_{-0.27}^{+0.24}$ & $0.40_{-0.20}^{+0.16}$ & $75 \pm 11$ & $159_{-13}^{+16}$ & $\mathrm{~W}+07$ \\
\hline
\end{tabular}

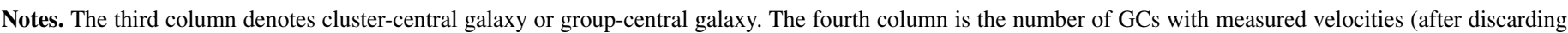
outliers). The seventh column shows the galaxy central velocity dispersion from HyperCat. ${ }^{18}$ http://leda.univ-lyon1.fr// (Paturel et al. 2003).

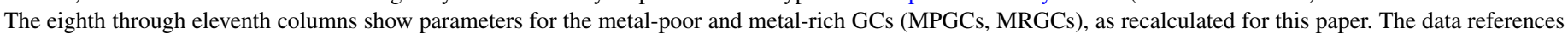

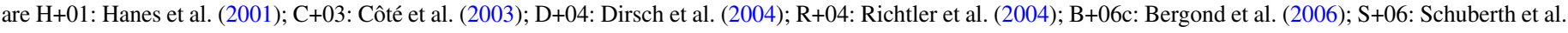
(2006); W+07: Woodley et al. (2007); L+08: Lee et al. (2008b).

To address these questions, we compile all of the published data available for GCS kinematics in group- and cluster-central galaxies (generically abbreviated as GCGs) with reasonably large and radially extended GC velocity catalogs (see Table 4). For the sake of consistency, we rederive the kinematical properties of these data sets using the same techniques that we have applied to NGC 1407. The redshifts of all the galaxies besides NGC 1407 are low enough to cause potential contamination from Galactic stars, which can easily distort the kinematical inferences by their accidental inclusion, or by overcompensating and excluding some low-velocity GCs. To be conservative, we have rejected all objects with velocities below $v_{\text {sys }}$ of the host galaxy. ${ }^{19}$

We discuss the GCS kinematics and dynamics in Section 5.1, the mass profiles in Section 5.2, the baryon content in Section 5.3, and some implications in Section 5.4.

\subsection{GCS Kinematics and Dynamics}

The kinematics of any galaxy should provide clues about its evolutionary history. However, GCGs have fairly similar optical properties to their more isolated elliptical galaxy counterparts (e.g., von der Linden et al. 2007; Bildfell et al. 2008; Loubser et al. 2008), which might reflect an early galaxy collapse history decoupled from the more recently assembled surrounding group. To probe the assemblies of both the GCGs and their groups, the large radial range of the GCs could be of service. The properties we will consider include the rotation (Section 5.1.1), velocity dispersion (Section 5.1.2), and orbits (Section 5.1.3) of GCs.

\subsubsection{Rotation}

We begin by examining GCS rotation in the galaxy sample, considering the metal-poor and the metal-rich rotational dominance parameter $\left(v_{\text {rot }} / \sigma_{\mathrm{p}}\right)$ and the kinematic misalignment angle $\left(\theta_{0 \text {,kin }}-\theta_{0 \text {,phot }}\right.$, where the $\pm 180^{\circ}$ degeneracy in $\theta_{0 \text {,phot }}$ is broken by reference to the direction of stellar rotation from the literature). These parameters are in general very dependent on the sampling of the GC velocity data by color, magnitude, and position - as illustrated in Table 2 and Figure 22 by the results for different radial bins of NGC 1407 GCs. However, momentarily throwing caution to the wind, we plot all of the available

\footnotetext{
19 A similar exercise for NGC 1407 yields rotation and kurtosis parameters that are formally inconsistent with the full data-set results, which is a warning that the uncertainties in the kinematical parameters are probably underestimated in general.
}

data from Table 4 (except for NGC 3379 that does not have enough GC velocities for this purpose).

These systems are generally consistent with an overall mild rotation of $v_{\text {rot }} / \sigma_{\mathrm{p}} \sim 0.2-0.6$ seen equally in both the metalpoor and metal-rich GCs (see also discussions in Beasley et al. 2006; Woodley et al. 2007; and H+08). The kinematic position angles of the metal-poor and metal-rich GCs are in most cases consistent with each other, but with no preferred alignment relative to the galaxy isophotes (i.e., both majorand minor-axis rotation are found, as well as orientations inbetween). Such kinematic misalignments are unsurprising, since the photometric axis of a triaxial galaxy can be readily twisted in projection (e.g., Franx et al. 1991; Romanowsky \& Kochanek 1998).

The GCs also appear in some cases to be misaligned relative to the (central) stellar kinematics, even to the extent of counterrotation, which would imply an intrinsic kinematic misalignment. Furthermore, there are apparent kinematical twists with radius (see Section 3.2) - as in the case of M87, where such a twist may coincide with the onset of the $\mathrm{cD}$ envelope and reflect a transition to the group environment (see $\mathrm{C}+01$ ). Note however that a stellar/GC misalignment, or a kinematical twist within a population, does not necessarily imply radically decoupled subcomponents, since subtle shifts in the orbit populations with radius can dramatically alter the net angular momentum (e.g., Jesseit et al. 2007; van den Bosch et al. 2008).

Deciphering the implications for the intrinsic structure and kinematics of these galaxies and their GC systems will require improved data and in-depth modeling. But our current overall impression is that the metal-poor and metal-rich GC subsystems have similar rotational properties, which might be distinct from the rotation of the field stars.

Considering next the connections to galaxy formation theory, we plot in Figure 22 (left) a theoretical prediction for the rotation amplitude from $\mathrm{B}+05$, as the median value and spread inside $6 R_{\text {eff }}$ of simulations of dissipationless disk galaxy pair major mergers (their multiple merger results appear to be fairly similar). Broadly speaking, their predictions $\left(v_{\text {rot }} / \sigma_{\mathrm{p}} \sim 0.4\right.$ for both metallicity subsystems) agree with the data. We will leave it as a future exercise to check their prediction that the rotation increases with radius.

$\mathrm{B}+05$ also predicted that the GCs would have kinematic misalignments relative to the galaxy isophotes, with similar rotation axes for the metal-rich and metal-poor subsystems-again in 

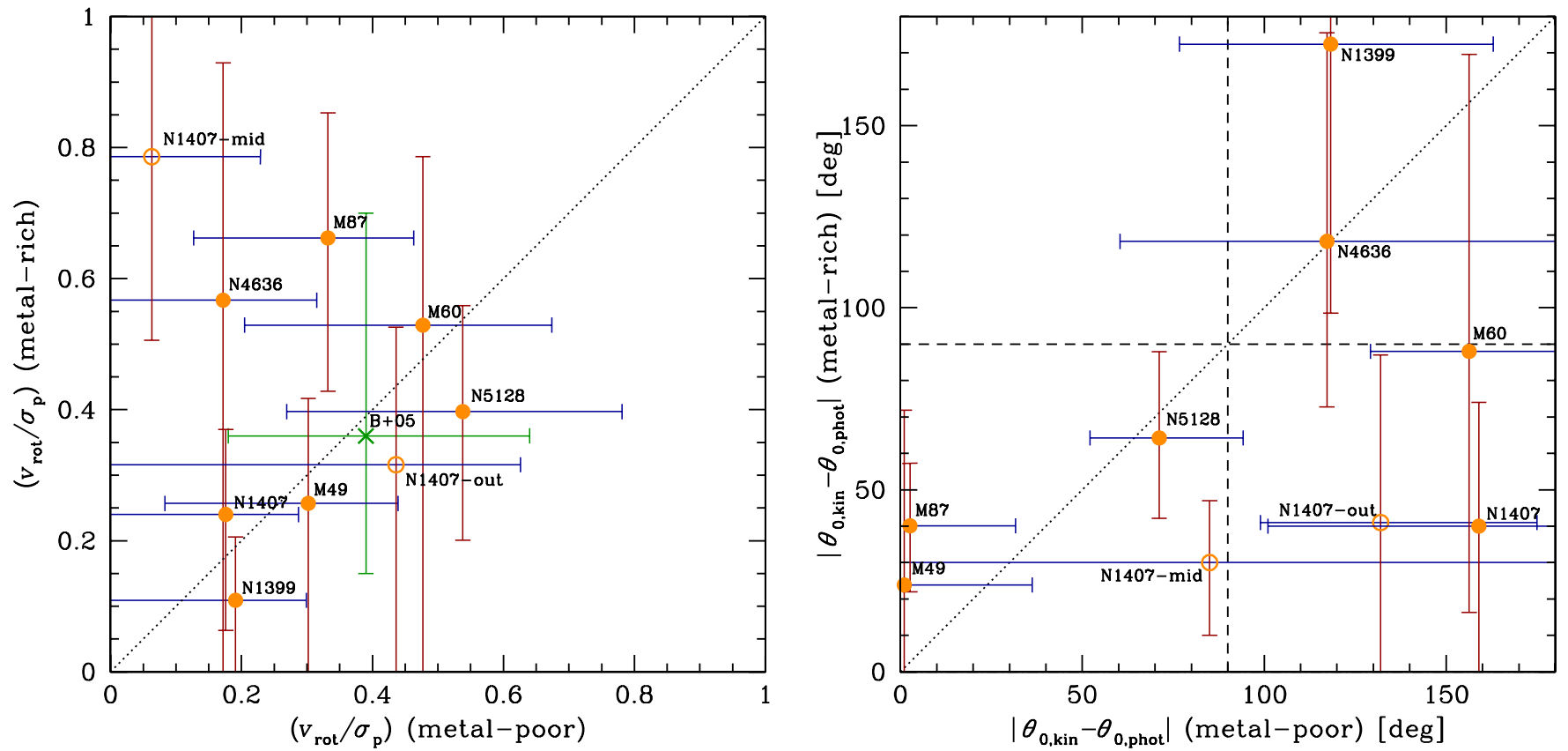

Figure 22. GCS rotation parameters for group/cluster-central galaxies, with the metal-poor and metal-rich subsystems compared. In the case of NGC 1407, separate points are plotted for the overall GCS, for intermediate radii (1'.77-4'.52), and for outer radii (4'.55-11'1). The dotted diagonal lines mark equal values for the subsystems. Left: rotation amplitude relative to velocity dispersion. The $(x)$ marks the results of the merger simulations of B+05. Right: kinematic misalignment. The angles $\theta_{0, \text { kin }}$ and $\theta_{0 \text {,phot }}$ correspond to the angular momentum vector and to the photometric minor axis, respectively. Classic rotation around the minor axis is at $0^{\circ}$, rotation along it at $90^{\circ}$ (dashed lines), and counter-rotation at $180^{\circ}$.

(A color version of this figure is available in the online journal.)

broad agreement with the observations. It is not clear whether kinematic twists with radius, or kinematic misalignments between the stars and GCs, are expected from these merger models. Intriguingly, B+05 also found that the rotation of a GC system aligns with the rotation of its host DM halo, potentially providing an indirect probe of an otherwise inaccessible property.

Detailed kinematics predictions do not exist for other GCS formation scenarios, such as accretion, quasi-monolithic multimode galaxy collapse, and metal-rich GC formation by major mergers - but with all of these scenarios, one would probably expect a difference between the rotation of the metal-poor and metal-rich GCs that is not seen in the data (see discussion in $\mathrm{H}+08$ ). This observation suggests that the GCs were in general formed prior to the assembly of their host GCG galaxies by mergers - which affected the metal-rich and metal-poor GCs equally, replacing any pre-existing orderly rotation with mergerinduced hot rotation. A related implication is that the last major merger episodes in these galaxies were "dry" or "damp" (relatively gas-poor; Forbes et al. 2007), as also suggested by other studies (De Lucia \& Blaizot 2007; Liu et al. 2008; McIntosh et al. 2008; Tran et al. 2008)

Such a scenario of early GC formation was considered as part of a recent foray into the modeling of GCGs in a cosmological context (Bekki et al. 2008). Their prediction of relatively low GCS rotation overall $\left(v_{\text {rot }} / \sigma_{\mathrm{p}} \lesssim 0.3\right)$ appears to conflict with the observations, but the simulations did not directly include nongravitational processes, which could alter the rotation predictions. Also, their result reflects all of the GCs within the virial radius, while the observations sample the GCs within only $\sim 0.1 r_{\text {vir }}$.

It will be interesting to see if a different rotation pattern emerges from observations of noncentral ellipticals (e.g., B. Kumar et al. 2010, in preparation), and of the general class of fainter, disky, rotation-dominated ellipticals and lenticulars (e.g., Emsellem et al. 2007). Such systems may have undergone more sporadic merger histories involving different mass ratios and more recent $\mathrm{GC}$ formation, and may have experienced more outer halo tidal stripping than GCGs have. Measurements of PN kinematics in all of these systems would also allow testing of the $\mathrm{B}+05$ prediction that the GCs and the field stellar halos should have kinematical similarities (broadly confirmed in the cases of NGC 5128 and M31; Peng et al. 2004; Woodley et al. 2007; Lee et al. 2008a).

To be robust, the theoretical predictions must also be improved to include baryonic effects in the mergers, and a full cosmological context.

\subsubsection{Velocity Dispersions}

We next compare the velocity dispersions in the GCG sample. In Figure 23, we have applied our smoothing technique to the data sets listed in Table 4 to produce GCS dispersion profiles. We note first of all that our NGC 1407 data out to $60 \mathrm{kpc}$ comprise the most radially extended set of GC velocities so far published for an early-type galaxy (a forthcoming data set for NGC 1399 will reach $80 \mathrm{kpc}$, as previewed in $\mathrm{R}+08$ ). Next we see that in GCGs a fairly constant profile is so far a generic property (see also the discussion in $\mathrm{H}+08$ ). Some departures from constancy are also evident, with hints in several galaxies of a central dispersion drop to $\sim 15-20 \mathrm{kpc}$, driven by the metalrich GCs (with NGC 1407 the most extreme case). M87 also stands out with its rapidly ascending dispersion. Although there is some indication in the NGC 1407 data of the onset of a large halo as in M87, the mass scales involved are considerably different (we will examine their mass profiles more directly in Section 5.2).

In the context of the $\mathrm{B}+05$ simulations, the fairly constant GC dispersion profiles in all of these systems would support their 


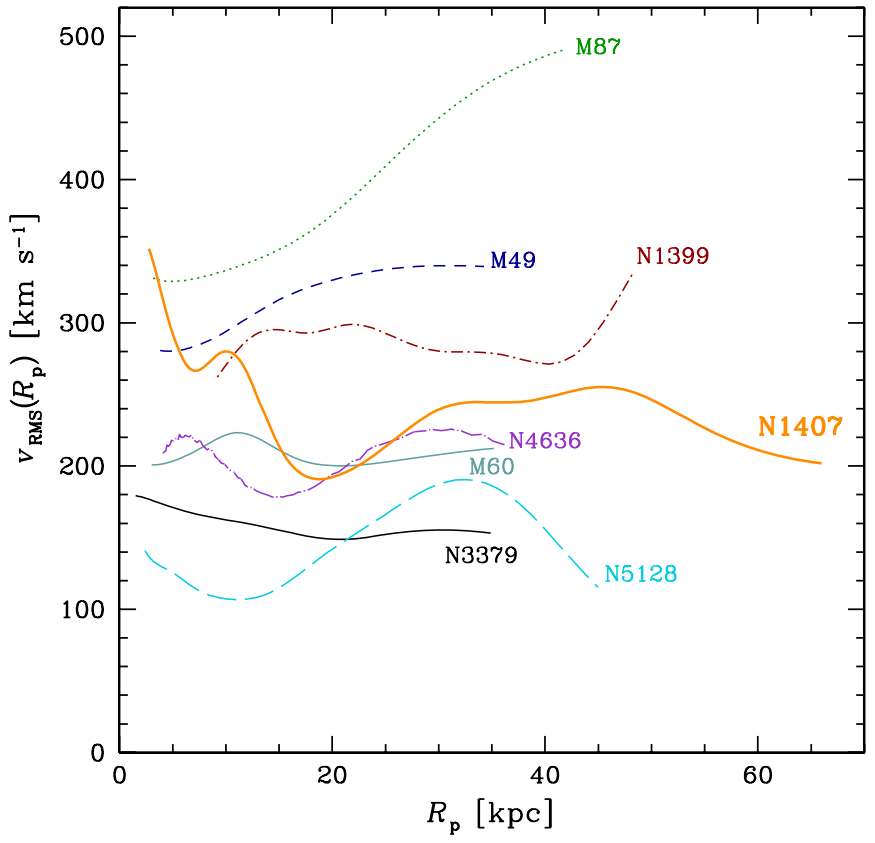

Figure 23. Projected, smoothed GCS rms velocity profiles of group- and cluster-central early-type galaxies. For clarity, average values are shown but no boundaries of uncertainty; typically the outermost $10 \%-20 \%$ of the radial range shown is based on few data points and the results there are not robust.

(A color version of this figure is available in the online journal.)

formation in multiple mergers, since pair mergers were found to have systematically more steeply declining dispersion profiles. This result in the simulations is presumably caused not by mass profile differences but by some combination of variations in the GCS spatial densities and orbital anisotropies. However, none of the B +05 merger remnants show a dispersion profile quite as flat as seen in the data-a discrepancy that could very well be due to more massive, extended DM halos around these GCGs. Interpreting the patterns seen in Figure 23 requires dynamical models to include the effects of mass, anisotropy, and spatial density (e.g., Section 5.2).

We next make a basic comparison between the global velocity dispersion amplitudes of the metal-poor and metal-rich GCs in these systems and note some interesting trends (Figure 24). The $v_{\text {rms }}$ of the metal-poor GCs seems to roughly track the central velocity dispersion of the host galaxy $\left(\sigma_{0}\right)$, while metalrich GCs may have a weaker dependence on $\sigma_{0}$. One would naively expect the opposite, since the metal-poor GCs extend farther out into the group halo and should be less coupled to the central galaxy's properties. Clearer conclusions will require a more in-depth, homogeneous analysis of these and additional systems.

\subsubsection{Orbital Properties}

Lastly, we consider the dynamics of the GCSs, in the context of their orbital anisotropies. We have made a tentative determination for NGC 1407 that the overall GCS has somewhat tangential anisotropy, which is strongest for the metal-poor GCs, with near-isotropy for the metal-rich GCs (Section 4.1.4). Such studies are in their infancy, but there is an emerging consensus that the overall GCS anisotropies of group-central ellipticals are isotropic or modestly tangential (see overview in $\mathrm{H}+08$ ). To summarize the situation, we plot the kurtoses of the metalpoor and metal-rich systems in Figure 25. In all cases, both subpopulations are consistent with zero or negative kurtosis

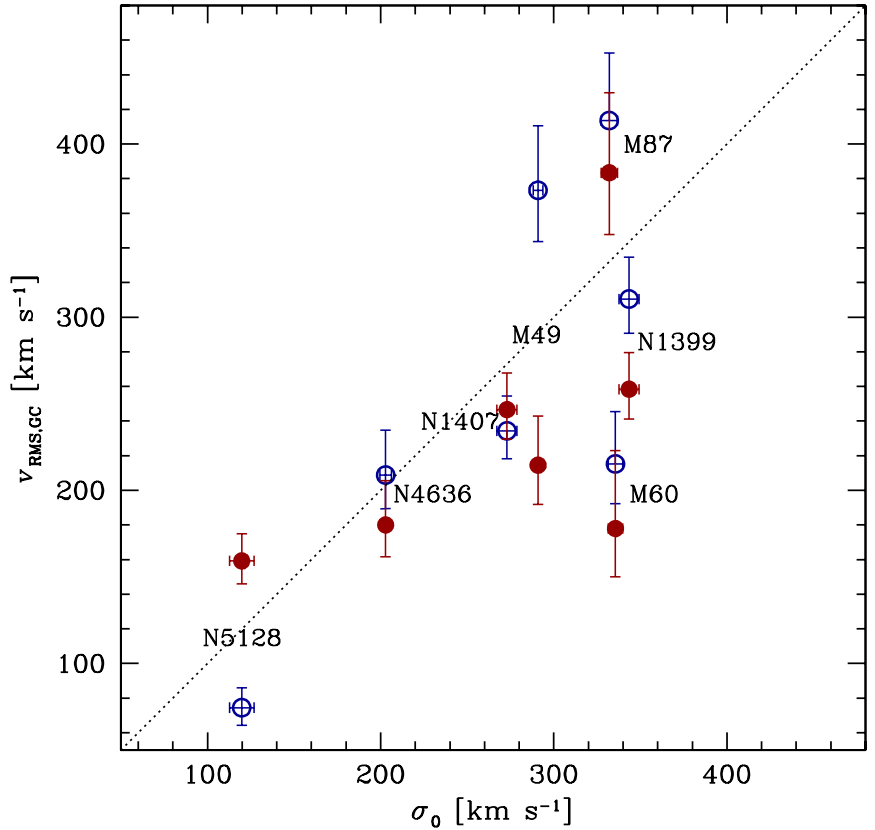

Figure 24. Overall GCS velocity dispersions vs. central galaxy velocity dispersions. The open blue circles show metal-poor subcomponents, and the filled red circles show metal-rich. The dotted line shows a one-to-one relation.

(A color version of this figure is available in the online journal.)

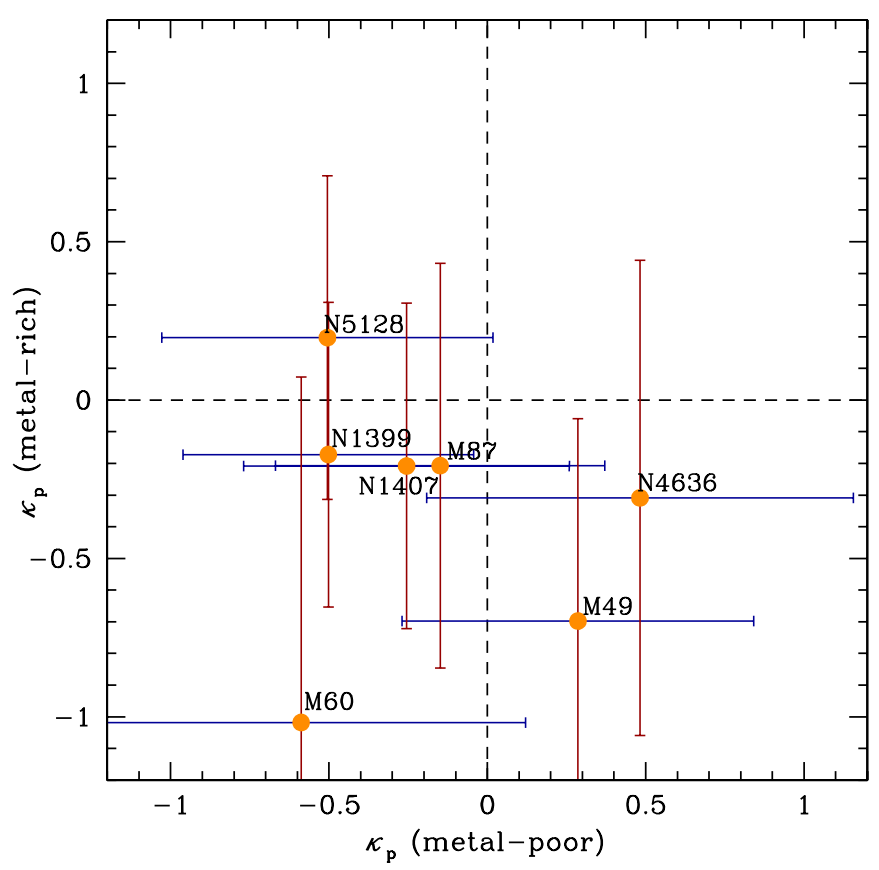

Figure 25. Kurtosis of the line-of-sight velocity distribution, for the metal-poor and metal-rich GC subpopulations of GCGs. The dashed lines mark Gaussian distributions $\left(\kappa_{\mathrm{p}}=0\right)$. Negative and positive kurtosis generally imply tangential and radial anisotropy, respectively.

(A color version of this figure is available in the online journal.)

$\left(\kappa_{\mathrm{p}}=-0.2 \pm 0.2\right.$ on average $)$, suggesting isotropic or somewhat tangentially anisotropic orbits.

This observational result conflicts with the general expectation from theory that stars and GCs in galaxy halos should be in somewhat radial orbits owing to infall and merger processes (see references in Section 4.1.3), a prediction that has been observationally confirmed for PNe (De Lorenzi et al. 2008, 2009; Napolitano et al. 2009). In particular, the results for the metal- 
poor GCs stand in stark contrast to the proposal of D+05 and Moore et al. (2006) that these GCs are associated with DM subsets on radial orbits (see Figure 13).

One caveat is that the theoretical predictions have generally considered individual galaxies rather than GCGs, which could in principle have distinctly different halo and GCS propertiese.g., perhaps the transition radius $r_{0}$ in Equation (6) would be much larger in such systems. ${ }^{20}$ There are so far no comparable data on more isolated galaxies, except for the Milky Way, where the metal-poor GC orbits are only mildly radial on average (van den Bosch et al. 1999).

The other key consideration is that the present-day population of GCs is probably a small surviving remnant of a primordial population that was subject to various destructive effects such as evaporation, dynamical friction, and tidal shocking (e.g., Gnedin \& Ostriker 1997; Fall \& Zhang 2001). In principle, these effects could be correlated with orbit type, so that GCs on radial orbits were preferentially destroyed, leaving a more isotropic or tangential distribution for present-day observers (e.g., Aguilar et al. 1988; Murali \& Weinberg 1997; Baumgardt 1998). However, a simulation of GCs in situ around an M87 analog indicated that orbit anisotropies are decreased only within a few kpc of the galaxy center (Vesperini et al. 2003) while the GCS anisotropies are observed to be low on scales of tens of kpc.

Still, there is much work remaining to be done for the predictions of initial GC orbits, and the connections of orbits with destruction. More realistic treatments of shocks and triaxial orbits may imply that GC disruption is more effective at larger radii (e.g., Dehnen et al. 2004; Capuzzo-Dolcetta \& Vicari 2005). Most importantly, the evolution of GCs should be followed in the context of a full cosmological picture of galaxy assembly. The only work along these lines so far is by Prieto \& Gnedin (2008), who found that tidal shocking is not a dominant effect in metal-poor GC evolution, and that the GC orbits are set by the orbits of their progenitor satellite galaxies (whether extant or disrupted), resulting in near-isotropy out to radii of $\sim 0.15 r_{\text {vir }}$, which is not too different from our observational constraints (Figure 13). However, the GCSs in these simulations are much more spatially extended than real systems, calling into question their mechanism for depositing the GCs and thus their anisotropy predictions (compare Abadi et al. 2006). Furthermore, the simulations describe an isolated Milky Way counterpart rather than a GCG, where the violent merging process should erase whatever initial orbits the GCs had anyway, leaving again the radial orbit puzzle discussed above.

Keeping in mind that the disruption rates of GCs may depend on their masses and concentrations, one wonders whether there is any signature dependence of GC kinematics with their luminosities or sizes. In fact, there are only a few galaxies where this issue has been examined: NGC 1399, NGC 4374, and NGC 4636 (R+04; Schuberth et al. 2006; B. Kumar et al. 2010, in preparation), and now NGC 1407 (see Section 3.4). In each case, a plot of GC velocities with magnitude reveals that the very brightest objects have noticeably platykurtotic distributions and correspondingly higher dispersions than the rest of the GCs. These features are not statistically significant in all cases (including NGC 1407), but the overall pattern is clear. One explanation could be that the population of bright GCs on radial orbits was depleted by disruption effects, leaving them

\footnotetext{
20 The simulations of GCG halo stars by Sommer-Larsen (2006) predict anisotropies that are very similar to the D+05 model in Figure 13 (GR, XR) in the case of fossil groups, and slightly less radial in the case of nonfossils.
}

with only near-circular orbits. This does not necessarily mean that the brightest GCs were most sensitive to these effects, since a general reduction in mass for all GCs on radial orbits would shift them toward lower luminosities, leaving the bright end of the distribution as the only subpopulation observably depleted of radial orbits.

In NGC 1407, we also see peculiar behavior at small radii, where the metal-rich GCs have a sharply increasing velocity dispersion that could imply a radial orbit distribution (see Sections 3.3 and 4.3.2). This conclusion seems to contradict the generic theoretical expectation that central GCs will tend to more tangential orbits, as high-velocity radially plunging objects would be destroyed near their pericenters. The presence of the DGTO candidates (Section 3.1) suggests another possibility: that some of the "normal" central GCs are really DGTOs that were formed from the nuclei of tidally stripped galaxies (e.g., Bekki et al. 2001; Fellhauer \& Kroupa 2006; Bekki 2007; Goerdt et al. 2008). These DGTOs would now have radially biased orbits, while a population of tangentially biased progenitor galaxies should linger at larger radii, for the same reasons discussed above for normal GC disruption. Whether the detailed anisotropy profiles from this theoretical scenario and the NGC 1407 observations are consistent, and whether the progenitor population can be found, remains to be seen. Other open questions are why there would be no signature of a residual central DGTO population in the GC kinematics of other GCGs, and why the DGTO population around NGC 1399 is observed to have a decreasing velocity dispersion toward the center (Thomas et al. 2008).

\subsection{Mass Profiles}

We next examine the mass profiles of the systems from Table 4 (we have not rederived these profiles as we did for the kinematics properties). For the sake of homogeneity, these include only results where the halo masses are constrained by the GCs rather than by any independent constraints such as PNe and X-rays. $\mathrm{X}$-ray studies in particular could be selection-biased toward systems with unusually massive, concentrated DM halos (e.g., Vikhlinin et al. 2006; Buote et al. 2007; Fedeli et al. 2007), since the resulting high X-ray luminosities of such systems are tempting observational targets. The GC studies so far may also have a similar, less direct bias, since the galaxies hosting large, readily studied GC populations are also likely to have more massive DM halos (Spitler et al. 2008b). Such a bias suits our current analysis of GCGs, but more general conclusions about halo masses in elliptical galaxies will require a careful galaxy sample selection independent of the GC content (e.g., B. Kumar et al. 2010, in preparation).

All of these GC-based mass results involved near-isotropic GC systems; we use model GT for NGC 1407 (see Section 4.1.4) rather than the consensus model C. In the case of M87, the RK01 model has been used rather than the mass model of $\mathrm{C}+01-$ which attempts to fit the overall Virgo cluster dynamics but does not match well the steep profile increase in the region constrained by the X-rays and GCs. We do not include NGC 3379 and M60 since clear results are not yet available based on the GCs alone. ${ }^{21}$

The summary of these results is shown in Figure 26. In most of these systems, an upturn in $v_{\mathrm{c}}(r)$ is visible: this is an intrinsic

\footnotetext{
21 For NGC 3379, the GCs are compatible with the PN-based result that the $v_{\mathrm{c}}(r)$ profile declines rapidly with radius (Bergond et al. 2006; Douglas et al. $2007)$, while for M60, the X-ray results imply a roughly constant $v_{\mathrm{c}}(r)$ profile to $\sim 30 \mathrm{kpc}$ (see $\mathrm{H}+08)$.
} 


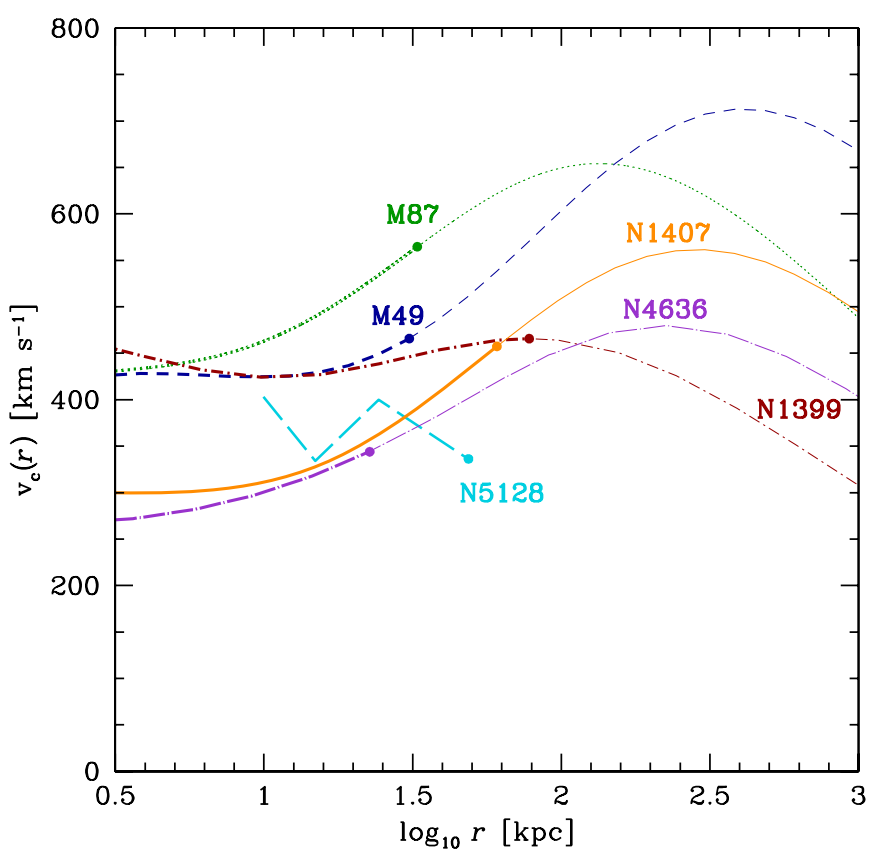

Figure 26. Mass modeling results for group/cluster-central galaxies, based on GCS dynamics. For clarity, no uncertainties are shown. The line styles correspond to galaxies as in Figure 23. The thicker segments of the curves with terminating points show the approximate radial range constrained by the data.

(A color version of this figure is available in the online journal.)

feature of $\Lambda \mathrm{CDM}$ halos that is masked in the central parts by the mass contribution of the galaxy, where it conspires to produce a roughly constant $v_{\mathrm{c}}(r)$ profile (see Gerhard et al. 2001; Rusin et al. 2003; Gavazzi et al. 2007). In most cases, the upturn is mild at the radii probed by the GC data, but in the cases of NGC 1407 and M87, a strong upturn is seen. Note that the $v_{\text {rms }}\left(R_{\mathrm{p}}\right)$ profiles (compare Figure 23) are not straightforward indicators of the $v_{\mathrm{c}}(r)$ profiles, which can be inferred only with a dynamical modeling process that includes the slopes of the tracer density profiles and (ideally) the tracer orbit anisotropies.

Before comparing NGC 1407 and M87 further, we note that their best-fit GC-based mass profiles are not unique, and in fact the two DM halo profiles are nearly consistent within the uncertainties. However, the independent constraints from $\mathrm{X}$ rays (for M87) and group galaxies (for NGC 1407) do support these best-fit models. The difference in $v_{\mathrm{c}}$ amplitudes between the two systems implies a factor of 2 difference in DM content within $\sim 30 \mathrm{kpc}$ (consistent with the observation that the $\mathrm{X}$ ray temperatures differ by a factor of 2). The M87 subcluster appears to have a much higher halo concentration than the NGC 1407 group, which suggests that the NGC 1407 group collapsed at a much lower redshift than the core of the Virgo cluster did.

To first order, a CDM halo's initial collapse redshift $z_{\mathrm{c}}$ is related to its characteristic density $\rho_{s}$ (tabulated for NGC 1407 in Table 3) by $\rho_{s} \propto\left(1+z_{\mathrm{c}}\right)^{3}$ (Navarro et al. 1997; Bullock et al. 2001). In more detail, the $\Lambda$ CDM toy model of Bullock et al. (2001) implies $z_{\mathrm{c}} \sim 1.7$ for NGC 1407 (age $\sim 10$ Gyr) and $z_{\mathrm{c}} \sim 4$ for M87, assuming no alteration of the central DM density through baryonic effects. ${ }^{22}$ The halo mass profile of NGC 1399 is still fairly uncertain (see R+08), but the bestguess central mass profile implies $z_{\mathrm{c}} \sim 4$. These numbers also reflect our result of a "normal" DM halo concentration for NGC

\footnotetext{
22 Trentham et al. (2006) used different criteria to suggest $z_{\mathrm{c}} \sim 0.8$ for the NGC 1407 group, while the simulations of von Benda-Beckmann et al. (2008) predict $z_{\mathrm{c}} \sim-0.1$ for NGC 1407, and $z \sim 0.05$ for M87 and NGC 1399 .
}

1407, while the other systems have fairly high concentrations, since at fixed mass, higher concentration halos will collapse first. (Note that if the X-ray result for NGC 1407 is correct, then it has $z_{\mathrm{c}} \sim 5$.) Optically, the Fornax core seems somewhat more relaxed than Virgo, with a higher galaxy density (also somewhat higher than that of the NGC 1407 group; B+06b; Jordán et al. 2007), suggesting an earlier collapse-but the DM density should provide the more accurate picture. Thus, the NGC 1407 group appears to be at an earlier evolutionary stage than both Virgo and Fornax (i.e., starting its collapse later).

\subsection{Baryon Content}

We next consider some global baryonic properties of the NGC 1407 group, comparing them to empirical and theoretical values for large samples of groups in the universe. We start with the average overall mass-to-light ratio $\Upsilon$ for galaxy groups. There are many possible sources for these values, most of them with similar findings, and we select the results of Eke et al. (2006), who used two different techniques to derive group and cluster $\Upsilon$ values based on $\sim 100,0002 \mathrm{dF}$ Galaxy Redshift Survey (2dFGRS) galaxies at $z \sim 0.1$ (their halo mass definitions are within a few percent of ours for $\Omega_{M}=0.3$ ). The first is a direct empirical technique of using the measured redshifts to calculate the masses of the individual galaxy groups with a virial analysis. The second is a hybrid empirical-theoretical matching of the observed luminosity function of groups to the mass function expected from $\Lambda C D M$ (we are not aware of any purely theoretical prediction, owing to the highly uncertain effects of baryonic physics in structure formation). As displayed in Figure 27, both approaches yield very similar results, with $\Upsilon$ increasing from a value of $\sim 60 \Upsilon_{\odot, b_{J}}$ for group luminosities of $L^{*}$ to a maximum value of $\sim 300 \Upsilon_{\odot, b_{J}}$ for clusters, ${ }^{23}$ which is similar to the average $\Upsilon$ for the whole universe.

We plot the results for the NGC 1407 group, increasing the $B$-band luminosity by $10 \%$ to map to the photographic $b_{J}$-band (see Eke et al. 2004b). Here we see that at the optical luminosity of the NGC 1407 group, a typical $\Upsilon$ ought to be $\sim 130-190 \Upsilon_{\odot, b_{J}}$. Our consensus solution is much higher than this, with $\Upsilon \sim 800$ $\Upsilon_{\odot, b_{I}}$, which is extreme even for a galaxy cluster. However, the range of uncertainty for NGC 1407 is still large, and we note that the shaded prediction region reflects the uncertainty in the mean value, not the cosmological scatter which may be larger. The NGC $1407 \Upsilon$ values could be brought down if more luminosity were included, e.g., in additional group member galaxies, extended stellar halos, or intragroup light. However, any additional members will be dwarfs and not contribute much to the total luminosity, while the latter two issues should affect the $2 \mathrm{dFGRS}$ survey results similarly. Note that in the $B$ - and $K$-bands, the NGC 1407 group $\Upsilon$ is $850_{-340}^{+1010} \Upsilon_{\odot, B}$ and $190_{-80}^{+300}$ $\Upsilon_{\odot, K}$, respectively (for solution GG, where no X-ray constraints or concentration priors are applied, $\Upsilon$ is $\sim 60 \%$ higher).

The high $\Upsilon$ of the NGC 1407 group goes along with other unusual properties. The low luminosity for its halo mass means that it is also deficient in bright galaxies, at a level $\sim 2-3$ times lower than found observationally for typical low-redshift groups of similar mass (Yang et al. 2005; Hansen et al. 2009), although NGC 1407 appears to be an outlier at only the $\sim 1-2 \sigma$ level. The luminosity of the central galaxy NGC 1407 itself is also low at the $\sim 2 \sigma$ level for groups of its mass (Lin \& Mohr 2004; Yang et al. 2005; Brough et al. 2008; More et al. 2009; but see Conroy

\footnotetext{
${ }^{23}$ Mountrichas \& Shanks (2007) used a different analysis on the same data
} and found $\Upsilon$ higher by a factor of $\sim 5$. 


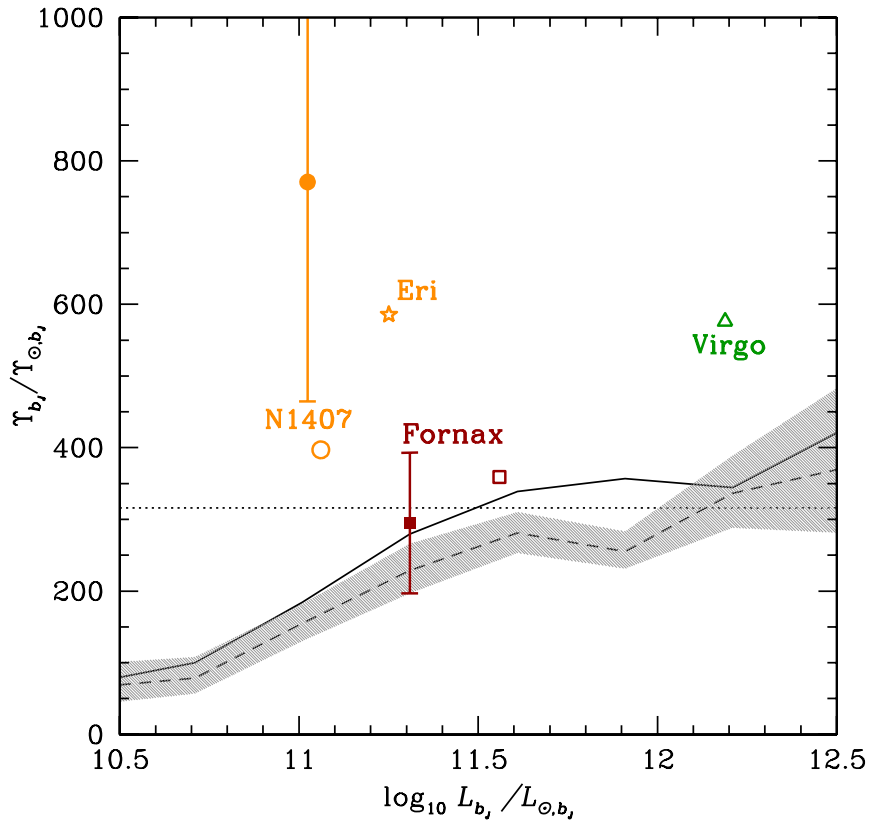

Figure 27. Virial mass-to-light ratio as a function of group luminosity. The dashed line with shaded region shows the empirical results (with uncertainties) from Eke et al. (2006) based on group dynamics. The solid line shows the semiempirical result from the same authors, based on luminosity-mass function matching. The horizontal dotted line shows the mean value for the universe, assuming a matter density of $\Omega_{\mathrm{M}}=0.3$; a Hubble constant of $H_{0}=70 \mathrm{~km} \mathrm{~s}^{-1} \mathrm{Mpc}^{-1}$ is adopted. The solid points with error bars show results for the NGC 1407 group (model C from this paper) and for the Fornax cluster (Drinkwater et al. 2001; R+08), based on models of their internal dynamics. The open points show more uniform results for the same systems and for the Virgo cluster, i.e., using the same survey data and mass analyses (from the projected mass estimator; Tully 2005). The star symbol shows a rough estimate for the eventual Eridanus supergroup/cluster, extrapolated from B+06b.

(A color version of this figure is available in the online journal.)

$\&$ Wechsler 2009). The group's early-type galaxy fraction of $\sim 80 \%-90 \%(\mathrm{~B}+06 \mathrm{a} ; \mathrm{B}+06 \mathrm{~b})$ is higher than typical for a group of its mass or velocity dispersion (Zabludoff \& Mulchaey 1998; Yang et al. 2005; B+06a). The only thing "normal" about the NGC 1407 group is the luminosity of the central galaxy relative to the total luminosity (OP04; Lin \& Mohr 2004; B+06a; Hansen et al. 2009).

For comparison, we also plot in Figure 27 some literature results for the two nearest galaxy clusters, Fornax and Virgo. These suggest that Fornax is more than twice as luminous as the NGC 1407 group, yet $\sim 30 \%$ less massive, resulting in a factor of $\sim 3$ difference in $\Upsilon$. Virgo on the other hand may have an $\Upsilon$ consistent with that of the NGC 1407 group, suggesting that these two systems are physically similar despite their very different galaxy content.

One key uncertainty in these conclusions is the distance: although this is ideally the era of distance measurements to $10 \%$ accuracy, NGC 1407 is a somewhat problematic case. As summarized by $\mathrm{S}+08$, distance estimates to this galaxy using various well-calibrated techniques range between 19 and $27 \mathrm{Mpc}$. We have adopted $21 \mathrm{Mpc}$ since larger distances would seemingly introduce problems for the GC luminosities and sizes (Forbes et al. 2006a), and incidentally would make it even harder to fit the X-ray data unless an implausibly low stellar $\Upsilon$ is adopted (see Section 4.3.1). Most of our results in this paper have been independent of distance, e.g., the shape of the $v_{\mathrm{c}}(r)$ profile, but changing from our default distance of $21 \mathrm{Mpc}$ to $27 \mathrm{Mpc}$ would increase the halo mass and shift
$\Upsilon$ downward (though still leaving NGC 1407 as an outlier in Figure 27).

An alternative way to consider the NGC 1407 group is via its baryon fraction $f_{\mathrm{b}} \equiv M_{\mathrm{b}} / M_{\mathrm{vir}}$, where $M_{\mathrm{b}}$ is the total baryon mass including stars (in and between galaxies), gas, and any other undefined baryonic form. Assigning a conservative uniform value of $\Upsilon_{*, B}=4.45 \Upsilon_{\odot, B}$ to all of the group galaxies, the total stellar mass is $\sim 4 \times 10^{11} M_{\odot}$; the hot gas mass is $\sim 10^{10} M_{\odot}(\mathrm{Z}+07)$. Our consensus mass result then implies that $f_{\mathrm{b}} \sim 0.002-0.007$. Given the cosmological $f_{\mathrm{b}}=0.17$ (Hinshaw et al. 2009), this means $\sim 96 \%-99 \%$ of the baryons in the system have been lost or else are invisible. Again, incorporating higher $\Upsilon_{*}$ values and "missed" galaxy halo light could boost $f_{\mathrm{b}}$, but only by a factor of $\sim 2$. Such a result would contradict theoretical expectations for cluster-mass halos to remain essentially closed boxes (e.g., De Lucia et al. 2004; Ettori et al. 2006; Crain et al. 2007), and observational evidence for baryon "disappearance" to be relatively constant at $\sim 25 \%$ (Gonzalez et al. 2007).

The underrepresentation of baryons may occur in many stellar systems (McGaugh 2008), which might be explained by expulsion from feedback mechanisms-even in systems as massive as NGC 1407 (e.g., Finoguenov et al. 2001). Intragroup stellar light could also be considered a candidate for the missing baryons, except that this would comprise $\sim 40$ times as much stellar light as the rest of the group galaxies combined. A third alternative is that most of the missing baryons reside in a relatively cool and/or diffuse gaseous medium (e.g., Cen \& Ostriker 2006; Nicastro et al. 2008), which upon collapse of the supergroup into a cluster might heat up to emission levels detectable by X-ray telescopes.

\subsection{Implications}

Bringing together all of the strands from the preceding subsections, we consider their possible implications for the evolutionary history of NGC 1407 and its group, and where this system fits in a cosmological context. Revisiting first the membership status of NGC 1400 with its large peculiar velocity, we note that there is no evidence from photometry and kinematics that it is associated with a second group of galaxies superimposed along the line of sight. Also, the high inferred group mass does not hinge upon this single velocity, and exceeds the minimum value $\left(\gtrsim 3 \times 10^{13} M_{\odot}\right)$ necessary to keep NGC 1400 bound. Interestingly, if NGC 1400 is now at pericenter on a near-radial orbit, then our best-fit solution "GT" implies that its apocenter is at $r_{\text {vir }}$, and thus that this galaxy has only recently joined the group (as also suggested by $\mathrm{S}+08$ ).

Without this new arrival, NGC 1407 would technically meet the definition for a fossil group, i.e., a system that consists optically of a single bright galaxy but has a group-mass halo (e.g., Jones et al. 2003). The implication is that the GCG swallowed up any other bright primordial members at early times, resulting in a transient fossil phase as experienced by many groups (D’Onghia et al. 2005; Dariush et al. 2007; von Benda-Beckmann et al. 2008).

The GC kinematics in NGC 1407 and other GCGs (i.e., the moderate amount of rotation and flat velocity dispersion profiles) appear broadly consistent with the formation of these galaxies in multiple major mergers of gas-free galaxies with pre-existing GCSs (Section 5.1). However, the involvement of the group environment is unclear, and more observational and theoretical work is needed to determine, e.g., when the GCGs formed relative to their surrounding groups, and whether the GCSs are more closely associated with the galaxies or the groups 
(compare Blakeslee et al. 1997; McLaughlin 1999; Spitler et al. 2008b).

The detailed mass profiles derived from the GC kinematics generally show an onset of the group- or cluster-scale halo via an increasing $v_{\mathrm{c}}(r)$. A comparison of the DM densities around NGC 1407, M87, and NGC 1399 implies that the cores of the Virgo and Fornax clusters collapsed at earlier times than the NGC 1407 group did. Some commonalities in the GC kinematics and mass profiles of NGC 1407 and M87 suggest that the formational pathways of these two systems were similar, albeit at different redshifts. The properties of the DM itself could in principle be further probed via the central slopes of the mass profiles and from deriving a mass-concentration relation-but any such conclusions will require greater care in dealing with selection effects, and with systematic issues such as orbital anisotropy.

Comparing the NGC 1407 group's global properties to empirical results for large samples of groups, we find that its high $\Upsilon$ and correspondingly low star formation make it a "dark cluster" as proposed by Gould (1993). The overall impression is of a poor galaxy group trapped in a high-mass halo that was somehow unable to form more galaxies, and which evolved its galaxies quickly to the red sequence owing to interactions or to the lack of fresh gas for star formation. The system could represent the tail end of galaxy cluster formation in the universe (with its peak at $z \sim 0.7$ for masses of $\sim 10^{14} M_{\odot}$; Harker et al. 2006; Conroy \& Wechsler 2009). The future collapse of the Eridanus "supergroup" into a cluster should bring the system's $\Upsilon$ closer to typical values (see Figure 27 ), while the missing baryons might emerge as the hypothetical unseen gas is heated into X-ray bright gas. The only incongruity would be the unusually low luminosity of NGC 1407 as a cluster-central galaxy, unless it experiences another major merger.

One could posit that high- $\Upsilon$ clusters such as Virgo were built up from progenitor groups like NGC 1407 with high $\Upsilon$ (but earlier collapse times), akin to the notion that many brightest cluster galaxies originated as fossil groups (Khosroshahi et al. 2006). This scenario has intriguing relevance for the observational puzzle that the stellar mass fraction $f_{*}$ in low-redshift galaxy groups and clusters decreases with mass-contradicting the expectation from CDM hierarchical merging that more massive systems are formed from less massive systems with similar $f_{*}$ (Balogh et al. 2008). The NGC 1407 group on the other hand has a very low $f_{*} \sim 0.004$, which would go in the direction needed by theory. An obvious interpretation is that this group is a relic from an earlier epoch, when the progenitor groups of clusters had systematically lower $f_{*}$. However, as discussed by Balogh et al. (2008), the other present-day groups should also have low $f_{*}$ unless they had problematically recent bouts of star formation. One way out is to suppose that NGC 1407 represents the tip of an iceberg, of groups that were either missed observationally, or had their masses systematically underestimated. A less mundane alternative is that structure formation on these scales is anti-hierarchical (e.g., because of warm DM).

The notion that clusters are built up from a biased subset of groups would also point to a preprocessing scenario for cluster galaxy evolution. However, the trend of recent findings is that galaxy clusters have fairly universal properties related to their total mass rather than to their merger histories, i.e., the galaxy content is somehow systematically altered as the clusters are assembled, rather than the clusters being assembled from systematically distinct groups (e.g., Lin et al. 2006; Muzzin et al. 2007; Hansen et al. 2009). Additional nuances are being added to this picture (e.g., Weinmann et al. 2006; Poggianti et al. 2006), and it is possible that a mass-correlated parameter such as density could be the main driving factor. In this context, the NGC 1407 group could be a telling system, since it is an outlier in the usual optical correlations, and its mass is below the proposed boundary for galaxy quenching to occur (Poggianti et al. 2006).

To better understand the implications of the NGC 1407 group, its uniqueness must be clarified. There are observational problems with detecting such systems, as discussed by Gould (1993): the "finger-of-God" effect of a high mass-poor group tends to be lost in the crowd of neighboring redshifts (see also Cowan \& Ivezić 2008). It would thus not be surprising if many such systems have been missed in optical surveys. On the other hand, the group is easily detected in X-ray surveys, with a rough $T_{\mathrm{X}}$-based guess for $\Upsilon$ that is only a factor of $\sim 2$ lower than our detailed estimate (see Section 4.2). Furthermore, it is an ordinary system when compared to X-ray correlations involving $L_{\mathrm{X}}, T_{\mathrm{X}}$, $\sigma_{\mathrm{p}}, \beta_{\mathrm{spec}}, \beta_{\mathrm{X}}, M_{\mathrm{vir}}, L_{B}$, etc. (O'Sullivan et al. 2001; OP04; $\mathrm{B}+06 \mathrm{a})$. Its only unusual aspect seems to be a $T_{\mathrm{X}}$ somewhat higher than typical for its $L_{\mathrm{X}}$, although the $T_{\mathrm{X}}(r)$ profile in detail may not be unusual for a group (Rasmussen \& Ponman 2007; compare Diehl \& Statler 2008a). This contrasts with its optical properties, almost all of which appear to be unusual (see Section 5.3). There may be something of a contradiction between optical- and X-ray-based mass functions which we cannot resolve in the present paper (compare Mendel et al. 2008).

There is anecdotal evidence for systems similar to the NGC 1407 group. The fairly small nearby group catalogs of Tully (2005) and B+06a include a few more candidates for ultra-dark groups-suggesting that the $\Upsilon$ of NGC 1407 may be well within the cosmological scatter. Two lower mass groups at intermediate redshift in the survey of Poggianti et al. (2006) were found to have unusually high early-type galaxy fractions, prompting the authors to dub them "bare" massive-cluster cores. The "dark core" in the cluster Abell 520 (Mahdavi et al. 2007) could be a preserved descendant of a group like NGC 1407, although this core appears to be missing its GCG. High- $\Upsilon$ systems could also be detected as "dark clumps" in weak lensing surveys (compare Erben et al. 2000; von der Linden et al. 2006).

Another fairly nearby group associated with NGC 1600 may also have $\Upsilon_{B} \sim 1000 \Upsilon_{B, \odot}$, as found by Smith et al. (2008), who suggest that this system represents a new class of fossil group with relatively low $L_{X}$. Alternatively, NGC 1407 and NGC 1600 might be heralds of a lurking population of massive ultra-dark groups, which were recognized in these cases only because of their bright GCGs and X-ray emission. A careful mining of modern observational surveys, and of cosmological simulations, may reveal the frequency of such systems, and their place within the wider context of structure formation.

\section{CONCLUSIONS}

We have used Keck/DEIMOS to study the kinematics of GCs around NGC 1407, arriving at a final sample (including literature data) of $172 \mathrm{GC}$ velocities out to galactocentric radii of $\sim 60 \mathrm{kpc}$. Outside the central regions, the GC system has weak rotation $\left(v_{\text {rot }} / \sigma_{p} \sim 0.2\right)$ around the major axis, with the metal-poor and metal-rich GC subsystems showing rotational misalignments. We also detect a moving group of GCs at large radii.

The GC velocity dispersion profile declines rapidly to $\sim 2^{\prime}$, whereafter it stays constant or rises gradually. The rapid decline 
may be due to a contribution of centrally located unresolved dwarf-globular transition objects on high-eccentricity orbits; we find two clear photometric examples of such objects. We also identify one probable intragroup GC, and find that the "blue tilt" (a color-magnitude relation for metal-poor GCs) discovered with HST imaging persists with ground-based photometry of a spectroscopically confirmed GC sample.

Dynamical modeling of the GCS indicates a circular velocity profile that continues to rise out to at least $60 \mathrm{kpc}$, consistent with a massive $\Lambda \mathrm{CDM}$ halo with $M_{\mathrm{vir}} \sim 7 \times 10^{13} M_{\odot}$ and a group mass-to-light ratio of $\Upsilon_{B} \sim 800 \Upsilon_{\odot, B}$. This agrees with results from group galaxy kinematics, but our preliminary $\mathrm{X}$ ray-based analysis of the mass profile shows some discrepancies. In order to reach agreement, the GCs would have to follow an implausible orbital distribution, with highly radial anisotropy in the center rapidly transitioning to tangential anisotropy in the outer parts. We survey X-ray results in other systems, finding some suggestions of discrepancies with kinematicsbased results. Also, the common phenomenon of X-ray "kinks" implies problems either with the assumption of hydrostatic equilibrium, with standard stellar $\Upsilon$ values, or with the CDM paradigm. Resolving this issue is of obvious importance to cosmological inferences from X-ray-based mass functions.

We compare NGC 1407 and other nearby group- and clustercentral galaxies, finding similarities in their GC kinematics and mass profiles. We suggest that the NGC 1407 group is similar to, but collapsed later than, the "seed" progenitor group that became the core of the Virgo cluster. Comparing its properties to large surveys of galaxy groups and clusters, this group appears to be a strong outlier. It is one of the "darkest" systems in the universe, whose $\Upsilon$ is similar to those of the faintest dwarf galaxies (Simon \& Geha 2007), and we are left with a puzzle as to why star formation was so attenuated in this system, and where the initial cosmological baryon complement is hiding. Finding more such high- $\Upsilon$ groups is part of the difficult but crucial inventory of mass and light in the universe, and could help disentangle the roles of halo density and mass in driving galaxy evolution.

Our new observations compound the existing evidence that GC systems have isotropic or tangentially biased velocity distributions, which seems to dramatically contradict most theoretical expectations for halo particles to reside on radially based orbits. However, there are open questions about the orbital dynamics of GC acquisition and destruction in a realistic theoretical context, and about possible observational links between GC luminosities, sizes, and orbits.

NGC 1407 and its surrounding galaxy group are an intriguing system that warrants further investigation. More complete kinematics observations and more detailed dynamical models are necessary to unravel its orbital and mass-to-light ratio mysteries. Our efforts continue along these lines.

We thank Jürg Diemand, Sandy Faber, Caroline Foster, Oleg Gnedin, Brijesh Kumar, Bill Mathews, Nicola Napolitano, Julio Navarro, Joel Primack, Simon White, and Marcel Zemp for helpful discussions, and Mark Wilkinson for a constructive review. We thank Eugene Churazov, Vince Eke, Yasushi Fukazawa, Fill Humphrey, Kyoko Matsushita, and Haiguang Xu for providing their results in tabular form, and Javier Cenarro, George Hau, Rob Proctor, and Max Spolaor for supplying their data to us prior to publication. Some of the data presented herein were obtained at the W. M. Keck Observatory, which is operated as a scientific partnership among the California Institute of Technology, the University of California, and the National
Aeronautics and Space Administration. The Observatory was made possible by the generous financial support of the W. M. Keck Foundation. The analysis pipeline used to reduce the DEIMOS data was developed at UC Berkeley with support from NSF grant AST-0071048. Based in part on data collected at Subaru Telescope (which is operated by the National Astronomical Observatory of Japan), via a Gemini Observatory time exchange (Program ID GC-2006B-C-18). We used methods and code provided in Numerical Recipes (Press et al. 1992). This material is based upon work supported by the National Science Foundation under grants AST-0507729 and AST-0808099, and by NASA grants HST-GO-09766.01-A and HST-GO-10402.06-A. A.J.R. was further supported by the FONDAP Center for Astrophysics CONICYT 15010003. J.S. was supported by NASA through a Hubble Fellowship, administered by the Space Telescope Science Institute, which is operated by the Association of Universities for Research in Astronomy, Incorporated, under NASA contract NAS5-26555. L.S. and D.F. thank the ARC for financial support.

Facilities: Keck:II, Keck:I, Subaru, HST

\section{REFERENCES}

Abadi, M. G., Navarro, J. F., \& Steinmetz, M. 2006, MNRAS, 365, 747

Aguilar, L., Hut, P., \& Ostriker, J. P. 1988, ApJ, 335, 720

Alam, S. M. K., Bullock, J. S., \& Weinberg, D. H. 2002, ApJ, 572, 34

Angus, G. W., Famaey, B., \& Buote, D. A. 2008, MNRAS, 387, 1470

Balogh, M. L., McCarthy, I. G., Bower, R. G., \& Eke, V. R. 2008, MNRAS, 385,1003

Barnes, J. 1985, MNRAS, 215, 517

Baumgardt, H. 1998, A\&A, 330, 480

Baumgardt, H., Kroupa, P., \& Parmentier, G. 2008, MNRAS, 384, 1231

Beasley, M. A., Strader, J., Brodie, J. P., Cenarro, A. J., \& Geha, M. 2006, AJ, 131,814

Bekki, K. 2007, MNRAS, 380, 1177

Bekki, K., Beasley, M. A., Brodie, J. P., \& Forbes, D. A. 2005, MNRAS, 363, $1211(\mathrm{~B}+05)$

Bekki, K., Couch, W. J., \& Drinkwater, M. J. 2001, ApJ, 552, L105

Bekki, K., Yahagi, H., Nagashima, M., \& Forbes, D. A. 2008, MNRAS, 387 , 1131

Benatov, L., Rines, K., Natarajan, P., Kravtsov, A., \& Nagai, D. 2006, MNRAS, 370,427

Benson, A. J., Bower, R. G., Frenk, C. S., Lacey, C. G., Baugh, C. M., \& Cole, S. 2003, ApJ, 599, 38

Bergond, G., Zepf, S. E., Romanowsky, A. J., Sharples, R. M., \& Rhode, K. L. 2006, A\&A, 448, 155 (B+06c)

Bernardi, M., et al. 2006, AJ, 131, 2018

Bildfell, C., Hoekstra, H., Babul, A., \& Mahdavi, A. 2008, MNRAS, 389, 1637

Binney, J., \& Tremaine, S. 1987, Galactic Dynamics (Princeton, NJ: Princeton Univ. Press), 747

Biviano, A., Murante, G., Borgani, S., Diaferio, A., Dolag, K., \& Girardi, M. 2006, A\&A, 456, 23

Blakeslee, J. P., Tonry, J. L., \& Metzger, M. R. 1997, AJ, 114, 482

Blumenthal, G. R., Faber, S. M., Flores, R., \& Primack, J. R. 1986, ApJ, 301, 27

Bridges, T., et al. 2006, MNRAS, 373, 157

Brighenti, F., \& Mathews, W. G. 1997, ApJ, 486, L83

Broadhurst, T. J., \& Barkana, R. 2008, MNRAS, 390, 1647

Broadhurst, T., Umetsu, K., Medezinski, E., Oguri, M., \& Rephaeli, Y. 2008, ApJ, 685, L9

Brodie, J. P., \& Strader, J. 2006, ARA\&A, 44, 193

Brough, S., Couch, W. J., Collins, C. A., Jarrett, T., Burke, D. J., \& Mann, R G. 2008, MNRAS, 385, L103

Brough, S., Forbes, D. A., Kilborn, V. A., \& Couch, W. 2006a, MNRAS, 370 , 1223 (B+06a)

Brough, S., Forbes, D. A., Kilborn, V. A., Couch, W., \& Colless, M. 2006b, MN RAS, 369, 1351 (B+06b)

Brownstein, J. R., \& Moffat, J. W. 2006, MNRAS, 367, 527

Bruzual, G., \& Charlot, S. 2003, MNRAS, 344, 1000

Bullock, J. S., Kolatt, T. S., Sigad, Y., Somerville, R. S., Kravtsov, A. V., Klypin, A. A., Primack, J. R., \& Dekel, A. 2001, MNRAS, 321, 559 
Buote, D. A., Gastaldello, F., Humphrey, P. J., Zappacosta, L., Bullock, J. S., Brighenti, F., \& Mathews, W. G. 2007, ApJ, 664, 123

Cappellari, M., et al. 2006, MNRAS, 366, 1126

Capuzzo-Dolcetta, R., \& Vicari, A. 2005, MNRAS, 356, 899

Carlberg, R. G., Yee, H. K. C., Morris, S. L., Lin, H., Hall, P. B., Patton, D. R., Sawicki, M., \& Shepherd, C. W. 2001, ApJ, 563, 736

Cen, R., \& Ostriker, J. P. 2006, ApJ, 650, 560

Cenarro, A. J., Beasley, M. A., Strader, J., Brodie, J. P., \& Forbes, D. A. 2007, AJ, 134, $391(\mathrm{C}+07)$

Chakrabarty, D., \& Raychaudhury, S. 2008, AJ, 135, 2350

Chanamé, J., Kleyna, J., \& van der Marel, R. 2008, ApJ, 682, 841

Churazov, E., Forman, W., Vikhlinin, A., Tremaine, S., Gerhard, O., \& Jones, C. 2008, MNRAS, 388, 1062 (C+08)

Ciotti, L., \& Pellegrini, S. 2004, MNRAS, 350, 609

Cohen, J. G. 2000, AJ, 119, 162

Cohen, J. G., \& Ryzhov, A. 1997, ApJ, 486, 230

Cole, S., \& Lacey, C. 1996, MNRAS, 281, 716

Colín, P., Klypin, A. A., \& Kravtsov, A. V. 2000, ApJ, 539, 561

Condon, J. J., Cotton, W. D., Greisen, E. W., Yin, Q. F., Perley, R. A., Taylor, G. B., \& Broderick, J. J. 1998, AJ, 115, 1693

Conroy, C., \& Wechsler, R. H. 2009, ApJ, submitted (arXiv:0805.3346)

Cooray, A., \& Milosavljević, M. 2005, ApJ, 627, L85

Côté, P., McLaughlin, D. E., Cohen, J. G., \& Blakeslee, J. P. 2003, ApJ, 591, $850(\mathrm{C}+03)$

Côté, P., et al. 2001, ApJ, 559, $828(\mathrm{C}+01)$

Cowan, N. B., \& Ivezić, Ž. 2008, ApJ, 674, L13

Crain, R. A., Eke, V. R., Frenk, C. S., Jenkins, A., McCarthy, I. G., Navarro, J. F., \& Pearce, F. R. 2007, MNRAS, 377, 41

Danese, L., de Zotti, G., \& di Tullio, G. 1980, A\&A, 82, 322

Dariush, A., Khosroshahi, H. G., Ponman, T. J., Pearce, F., Raychaudhury, S., \& Hartley, W. 2007, MNRAS, 382, 433

Dehnen, W., Odenkirchen, M., Grebel, E. K., \& Rix, H.-W. 2004, AJ, 127, 2753

De Lorenzi, F., Gerhard, O., Saglia, R. P., Sambhus, N., Debattista, V. P., Pannella, M., \& Méndez, R. H. 2008, MNRAS, 385, 1729

Dekel, A., \& Birnboim, Y. 2006, MNRAS, 368, 2

Dekel, A., Stoehr, F., Mamon, G. A., Cox, T. J., Novak, G. S., \& Primack, J. R. 2005, Nature, 437, 707

De Lorenzi, F., et al. 2009, MNRAS, 395, 76

De Lucia, G., \& Blaizot, J. 2007, MNRAS, 375, 2

De Lucia, G., Kauffmann, G., \& White, S. D. M. 2004, MNRAS, 349, 1101

Diaferio, A. 1999, MNRAS, 309, 610

Diehl, S., \& Statler, T. S. 2007, ApJ, 668, 150

Diehl, S., \& Statler, T. S. 2008a, ApJ, 687, 986

Diehl, S., \& Statler, T. S. 2008b, ApJ, 680, 897

Diemand, J., Madau, P., \& Moore, B. 2005, MNRAS, 364, 367 (D+05)

Dirsch, B., et al. 2004, AJ, 127, 2114

D’Onghia, E., Sommer-Larsen, J., Romeo, A. D., Burkert, A., Pedersen, K., Portinari, L., \& Rasmussen, J. 2005, ApJ, 630, L109

Douglas, N. G., et al. 2007, ApJ, 664, 257

Drinkwater, M. J., Gregg, M. D., \& Colless, M. 2001, ApJ, 548, L139

Drinkwater, M. J., Jones, J. B., Gregg, M. D., \& Phillipps, S. 2000, PASA, 17, 227

Duffy, A. R., Schaye, J., Kay, S. T., \& Dalla Vecchia, C. 2008, MNRAS, 390, L64

Eke, V. R., Baugh, C. M., Cole, S., Frenk, C. S., \& Navarro, J. F. 2006, MNRAS, 370,1147

Eke, V. R., et al. 2004a, MNRAS, 348, 866

Eke, V. R., et al. 2004b, MNRAS, 355, 769

Emsellem, E., et al. 2007, MNRAS, 379, 401

Erben, T., van Waerbeke, L., Mellier, Y., Schneider, P., Cuillandre, J.-C., Castander, F. J., \& Dantel-Fort, M. 2000, A\&A, 355, 23

Ettori, S., Dolag, K., Borgani, S., \& Murante, G. 2006, MNRAS, 365, 1021

Evstigneeva, E. A., Drinkwater, M. J., Jurek, R., Firth, P., Jones, J. B., Gregg, M. D., \& Phillipps, S. 2007, MNRAS, 378, 1036

Faber, S. M., et al. 2003, in Proc. SPIE 4841, Instrument Design and Performance for Optical/Infrared Ground-based Telescopes, ed. M. Iye \& A. F. M. Moorwood (Bellingham, WA: SPIE), 1657-1669

Fabricant, D., Lecar, M., \& Gorenstein, P. 1980, ApJ, 241, 552

Fall, S. M. 1983, in IAU Symp. 100, Internal Kinematics and Dynamics of Galaxies, ed. E. Athanassoula (Dordrecht: Kluwer), 391398

Fall, S. M., \& Zhang, Q. 2001, ApJ, 561, 751

Faltenbacher, A., \& Mathews, W. G. 2007, MNRAS, 375, 313

Fedeli, C., Bartelmann, M., Meneghetti, M., \& Moscardini, L. 2007, A\&A, 473, 715

Feldmann, R., Mayer, L., \& Carollo, C. M. 2008, ApJ, 684, 1062

Fellhauer, M., \& Kroupa, P. 2006, MNRAS, 367, 1577

Ferguson, H. C., \& Sandage, A. 1990, AJ, 100, 1
Finoguenov, A., Arnaud, M., \& David, L. P. 2001, ApJ, 555, 191

Firth, P., Evstigneeva, E. A., Jones, J. B., Drinkwater, M. J., Phillipps, S., \& Gregg, M. D. 2006, MNRAS, 372, 1856

Forbes, D. A., Proctor, R., Strader, J., \& Brodie, J. P. 2007, ApJ, 659, 188

Forbes, D. A., Sánchez-Blázquez, P., Phan, A. T. T., Brodie, J. P., Strader, J., \& Spitler, L. 2006a, MNRAS, 366, 1230

Forbes, D. A., et al. 2006b, PASA, 23, 38

Forte, J. C., Faifer, F., \& Geisler, D. 2007, MNRAS, 382, 1947

Franx, M., Illingworth, G., \& de Zeeuw, T. 1991, ApJ, 383, 112

Fujita, Y. 2004, PASJ, 56, 29

Fukazawa, Y., Botoya-Nonesa, J. G., Pu, J., Ohto, A., \& Kawano, N. 2006, ApJ, 636, 698

Fukushige, T., \& Makino, J. 2001, ApJ, 557, 533

Gao, L., Navarro, J. F., Cole, S., Frenk, C. S., White, S. D. M., Springel, V., Jenkins, A., \& Neto, A. F. 2008, MNRAS, 387, 536

Gastaldello, F., Buote, D. A., Humphrey, P. J., Zappacosta, L., Bullock, J. S., Brighenti, F., \& Mathews, W. G. 2007, ApJ, 669, 158

Gavazzi, R., Treu, T., Rhodes, J. D., Koopmans, L. V. E., Bolton, A. S., Burles, S., Massey, R. J., \& Moustakas, L. A. 2007, ApJ, 667, 176

Gerhard, O., Kronawitter, A., Saglia, R. P., \& Bender, R. 2001, AJ, 121, 1936

Ghizzardi, S., Molendi, S., Pizzolato, F., \& De Grandi, S. 2004, ApJ, 609, 638

Gilbank, D. G., \& Balogh, M. L. 2008, MNRAS, 385, L116

Girardi, M., Giuricin, G., Mardirossian, F., Mezzetti, M., \& Boschin, W. 1998, ApJ, 505, 74

Gnedin, O. Y., Kravtsov, A. V., Klypin, A. A., \& Nagai, D. 2004, ApJ, 616, 16 Gnedin, O. Y., \& Ostriker, J. P. 1997, ApJ, 474, 223

Goerdt, T., Moore, B., Kazantzidis, S., Kaufmann, T., Macciò, A. V., \& Stadel, J. 2008, MNRAS, 385, 2136

Gonzalez, A. H., Zaritsky, D., \& Zabludoff, A. I. 2007, ApJ, 666, 147

Gould, A. 1993, ApJ, 403, 37

Hanes, D. A., Côté, P., Bridges, T. J., McLaughlin, D. E., Geisler, D., Harris, G. L. H., Hesser, J. E., \& Lee, M. G. 2001, ApJ, 559, 812

Hansen, S. H., \& Moore, B. 2006, New Astron., 11, 333

Hansen, S. M., Sheldon, E. S., Wechsler, R. H., \& Koester, B. P. 2009, ApJ, submitted (arXiv:0710.3780)

Harker, G., Cole, S., Helly, J., Frenk, C., \& Jenkins, A. 2006, MNRAS, 367, 1039

Harris, W. E. 2009, ApJ, in press (arXiv:0904.4208)

Harris, W. E., Whitmore, B. C., Karakla, D., Okoń, W., Baum, W. A., Hanes, D. A., \& Kavelaars, J. J. 2006, ApJ, 636, 90 (H+06a)

Haşegan, M., et al. 2005, ApJ, 627, 203

Hilker, M., Infante, L., \& Richtler, T. 1999, A\&AS, 138, 55

Hinshaw, G., et al. 2009, ApJS, 180, 225

Hobbs, I. S., \& Willmore, A. P. 1997, MNRAS, 289, 685

Hobson, M. P., Bridle, S. L., \& Lahav, O. 2002, MNRAS, 335, 377

Humphrey, P. J., Buote, D. A., Gastaldello, F., Zappacosta, L., Bullock, J. S., Brighenti, F., \& Mathews, W. G. 2006, ApJ, 646, 899 (H+06b)

Hwang, H. S., \& Lee, M. G. 2008, ApJ, 676, 218

Hwang, H. S., et al. 2008, ApJ, 674, 869 (H+08)

Irwin, J. A., Athey, A. E., \& Bregman, J. N. 2003, ApJ, 587, 356

Jesseit, R., Naab, T., Peletier, R. F., \& Burkert, A. 2007, MNRAS, 376, 997

Joanes, D. N., \& Gill, C. A. 1998, The Statistician, 47, 183

Johnson, R., Chakrabarty, C., O’Sullivan, E., \& Raychaudhury, S. 2009, ApJ, submitted (J+09)

Jones, L. R., Ponman, T. J., Horton, A., Babul, A., Ebeling, H., \& Burke, D. J. 2003, MNRAS, 343, 627

Jordán, A., et al. 2007, ApJS, 169, 213

Kang, X., van den Bosch, F. C., \& Pasquali, A. 2007, MNRAS, 381, 389

Kautsch, S. J., Gonzalez, A. H., Soto, C. A., Tran, K.-V. H., Zaritsky, D., \& Moustakas, J. 2008, ApJ, 688, L5

Kawata, D., \& Mulchaey, J. S. 2008, ApJ, 672, L103

Khosroshahi, H. G., Ponman, T. J., \& Jones, L. R. 2006, MNRAS, 372, L68

Kirby, E., Jerjen, H., Ryder, S., \& Driver, S. 2008, in Galaxies in the Local Volume, ed. B. Koribalski \& H. Jerjen (Berlin: Springer) (arXiv:0709.2192)

Kissler-Patig, M., \& Gebhardt, K. 1998, AJ, 116, 2237

Kodama, T., \& Smail, I. 2001, MNRAS, 326, 637

Kronawitter, A., Saglia, R. P., Gerhard, O., \& Bender, R. 2000, A\&AS, 144, 53 Kundu, A. 2008, AJ, 136, 1013

Larsen, S. S., \& Richtler, T. 2000, A\&A, 354, 836

Lauer, T. R., et al. 1995, AJ, 110, 2622

Lee, M. G., Hwang, H. S., Kim, S. C., Park, H. S., Geisler, D., Sarajedini, A., \& Harris, W. E. 2008a, ApJ, 674, 886

Lee, M. G., et al. 2008b, ApJ, 674, 857

Lima Neto, G. B., Gerbal, D., \& Márquez, I. 1999, MNRAS, 309, 481

Lin, Y.-T., \& Mohr, J. J. 2004, ApJ, 617, 879

Lin, Y.-T., Mohr, J. J., Gonzalez, A. H., \& Stanford, S. A. 2006, ApJ, 650, L99 Liu, F. S., Xia, X. Y., Mao, S., Wu, H., \& Deng, Z. G. 2008, MNRAS, 385, 23 
Loubser, S. I., Sansom, A. E., Sánchez-Blázquez, P., Soechting, I. K., \& Bromage, G. E. 2008, MNRAS, 391, 1009

Ludlow, A. D., Navarro, J. F., Springel, V., Jenkins, A., Frenk, C. S., \& Helmi, A. 2009, ApJ, 692, 931

Maddox, S. J., Efstathiou, G., Sutherland, W. J., \& Loveday, J. 1990, MNRAS, 243, 692

Mahdavi, A., Hoekstra, H., Babul, A., Balam, D. D., \& Capak, P. L. 2007, ApJ, 668,806

Mahdavi, A., Hoekstra, H., Babul, A., \& Henry, J. P. 2008, MNRAS, 384, 1567

Maller, A. H., \& Bullock, J. S. 2004, MNRAS, 355, 694

Mamon, G. A., \& Łokas, E. L. 2005a, MNRAS, 362, 95

Mamon, G. A., \& Łokas, E. L. 2005b, MNRAS, 363, 705 (MŁ05)

Mathews, W. G., \& Brighenti, F. 2003, ApJ, 599, 992

Matsushita, K., Belsole, E., Finoguenov, A., \& Böhringer, H. 2002, A\&A, 386, 77

McGaugh, S. S. 2008, in IAU Symp. 244, ed. J. Disney \& M. Davies (Dordrecht: Kluwer), 136145

McIntosh, D. H., Guo, Y., Hertzberg, J., Katz, N., Mo, H. J., van den Bosch, F. C., \& Yang, X. 2008, MNRAS, 388, 1537

McLaughlin, D. E. 1999, AJ, 117, 2398

McMillan, P. J., Athanassoula, E., \& Dehnen, W. 2007, MNRAS, 376, 1261

Mendel, J. T., Proctor, R. N., Forbes, D. A., \& Brough, S. 2008, MNRAS, 389, 749

Merritt, D., \& Saha, P. 1993, ApJ, 409, 75

Merritt, D., \& Tremblay, B. 1993, AJ, 106, 2229

Mieske, S., et al. 2006, ApJ, 653, 193

Miyazaki, S., et al. 2002, PASJ, 54, 833

Monet, D. G., et al. 2003, AJ, 125, 984

Moore, B., Diemand, J., Madau, P., Zemp, M., \& Stadel, J. 2006, MNRAS, 368 , 563

Moran, S. M., Ellis, R. S., Treu, T., Smith, G. P., Rich, R. M., \& Smail, I. 2007, ApJ, 671, 1503

More, S., van den Bosch, F. C., Cacciato, M., Mo, H. J., Yang, X., \& Li, R. 2009, MNRAS, 392, 801

Mountrichas, G., \& Shanks, T. 2007, arXiv:0712.3255

Murali, C., \& Weinberg, M. D. 1997, MNRAS, 291, 717

Muzzin, A., Yee, H. K. C., Hall, P. B., \& Lin, H. 2007, ApJ, 663, 150

Nagai, D., Vikhlinin, A., \& Kravtsov, A. V. 2007, ApJ, 655, 98

Nagino, R., \& Matsushita, K. 2009, A\&A, submitted (arXiv:0903.2540)

Napolitano, N. R., Arnaboldi, M., \& Capaccioli, M. 2002, A\&A, 383, 791

Napolitano, N. R., et al. 2005, MNRAS, 357, 691

Napolitano, N. R., et al. 2009, MNRAS, 393, 329

Navarro, J. F., Frenk, C. S., \& White, S. D. M. 1996, ApJ, 462, 563

Navarro, J. F., Frenk, C. S., \& White, S. D. M. 1997, ApJ, 490, 493

Navarro, J. F., et al. 2004, MNRAS, 349, 1039

Neto, A. F., et al. 2007, MNRAS, 381, 1450

Nicastro, F., Mathur, S., \& Elvis, M. 2008, Science, 319, 55

Nipoti, C., \& Binney, J. 2007, MNRAS, 382, 1481

Nipoti, C., Londrillo, P., \& Ciotti, L. 2006, MNRAS, 370, 681

Norris, M. A., et al. 2008, MNRAS, 385, 40

Oke, J. B., et al. 1995, PASP, 107, 375

Oñorbe, J., Domínguez-Tenreiro, R., Sáiz, A., \& Serna, A. 2007, MNRAS, 376, 39

Osmond, J. P. F., \& Ponman, T. J. 2004, MNRAS, 350, 1511 (OP04)

O'Sullivan, E., Forbes, D. A., \& Ponman, T. J. 2001, MNRAS, 328, 461

O'Sullivan, E., Sanderson, A. J. R., \& Ponman, T. J. 2007, MNRAS, 380, 1409

Patel, S. G., Kelson, D. D., Holden, B. P., Illingworth, G. D., Franx, M., van der Wel, A., \& Ford, H. 2009, ApJ, 694, 1349

Paturel, G., Petit, C., Prugniel, P., Theureau, G., Rousseau, J., Brouty, M., Dubois, P., \& Cambrésy, L. 2003, A\&A, 412, 45

Pellegrini, S., \& Ciotti, L. 2006, MNRAS, 370, 1797

Peng, E. W., Ford, H. C., \& Freeman, K. C. 2004, ApJ, 602, 705

Perrett, K. M., Hanes, D. A., Butterworth, S. T., Kavelaars, J., Geisler, D., \& Harris, W. E. 1997, AJ, 113, 895

Pizzolato, F., Molendi, S., Ghizzardi, S., \& De Grandi, S. 2003, ApJ, 592, 62

Poggianti, B. M., et al. 2006, ApJ, 642, 188

Porter, A. C., Schneider, D. P., \& Hoessel, J. G. 1991, AJ, 101, 1561

Press, W. H. 1997, in Unsolved Problems in Astrophysics, ed. J. N. Bahcall \& J. P. Ostriker, (arXiv: astro-ph/9604126), 49-60

Press, W. H., Teukolsky, S. A., Vetterling, W. T., \& Flannery, B. P. 1992, Numerical Recipes in C: The Art of Scientific Computing (2nd ed; Cambridge: Cambridge Univ. Press)

Prieto, J. L., \& Gnedin, O. Y. 2008, ApJ, 689, 919

Pritzl, B. J., Venn, K. A., \& Irwin, M. 2005, AJ, 130, 2140

Proctor, R. N., Forbes, D. A., Brodie, J. P., \& Strader, J. 2008, MNRAS, 385 , 1709
Proctor, R. N., Forbes, D. A., Romanowsky, A. J., Brodie, J. P., Strader, J., Spolaor, M., Mendel, J. T., \& Spitler, L. 2009, MNRAS, submitted

Prugniel, P., \& Simien, F. 1997, A\&A, 321, 111

Quintana, H., Fouque, P., \& Way, M. J. 1994, A\&A, 283, 722

Rasia, E., Tormen, G., \& Moscardini, L. 2004, MNRAS, 351, 237

Rasia, E., et al. 2006, MNRAS, 369, 2013

Rasmussen, J., \& Ponman, T. J. 2007, MNRAS, 380, 1554

R Development Core Team 2006, R: A Language and Environment for Statistical Computing

Rees, M. J., \& Ostriker, J. P. 1977, MNRAS, 179, 541

Richtler, T., Schuberth, Y., Hilker, M., Dirsch, B., Bassino, L., \& Romanowsky, A. J. 2008, A\&A, 478, L23 (R+08)

Richtler, T., et al. 2004, AJ, 127, 2094

Rocca-Volmerange, B., Le Borgne, D., De Breuck, C., Fioc, M., \& Moy, E. 2004, A\&A, 415, 931

Romanowsky, A. J. 2006, in IAU Sym. 234, Planetary Nebulae in our Galaxy and Beyond, ed. M. J. Barlow \& R. H. Méndez (Dordrecht: Kluwer), 341

Romanowsky, A. J. 2009, in Globular Clusters: Guides to Galaxies, ed. T. Richtler \& S. Larsen (Berlin: Springer), 433

Romanowsky, A. J., Douglas, N. G., Arnaboldi, M., Kuijken, K., Merrifield, M. R., Napolitano, N. R., Capaccioli, M., \& Freeman, K. C. 2003, Science, 301, 1696

Romanowsky, A. J., \& Kochanek, C. S. 1998, ApJ, 493, 641

Romanowsky, A. J., \& Kochanek, C. S. 2001, ApJ, 553, 722 (RK01)

Rusin, D., Kochanek, C. S., \& Keeton, C. R. 2003, ApJ, 595, 29

Ryden, B. S. 1996, ApJ, 461, 146

Sales, L. V., Navarro, J. F., Abadi, M. G., \& Steinmetz, M. 2007, MNRAS, 379 , 1475

Samurovic, S. 2006, Serbian Astron. J., 173, 35

Samurović, S., \& Ćirković, M. M. 2008, A\&A, 488, 873

Sand, D. J., Treu, T., Smith, G. P., \& Ellis, R. S. 2004, ApJ, 604, 88

Sanderson, A. J. R., Ponman, T. J., \& O'Sullivan, E. 2006, MNRAS, 372, 1496

Schlegel, D. J., Finkbeiner, D. P., \& Davis, M. 1998, ApJ, 500, 525

Schmidt, R. W., \& Allen, S. W. 2007, MNRAS, 379, 209

Schuberth, Y., Richtler, T., Bassino, L., \& Hilker, M. 2008, A\&A, 477, L9

Schuberth, Y., Richtler, T., Dirsch, B., Hilker, M., Larsen, S. S., Kissler-Patig, M., \& Mebold, U. 2006, A\&A, 459, 391

Sérsic, J. L. 1968, Atlas de galaxias australes (Cordoba, Argentina: Observatorio Astronomico)

Sharples, R. M., Zepf, S. E., Bridges, T. J., Hanes, D. A., Carter, D., Ashman, K. M., \& Geisler, D. 1998, AJ, 115, 2337

Simon, J. D., \& Geha, M. 2007, ApJ, 670, 313

Smith, R. M., Martínez, V. J., Fernández-Soto, A., Ballesteros, F. J., \& Ortiz-Gil, A. 2008, ApJ, 679, 420

Sommer-Larsen, J. 2006, MNRAS, 369, 958

Spitler, L. R., Forbes, D. A., \& Beasley, M. A. 2008a, MNRAS, 389, 1150

Spitler, L. R., Forbes, D. A., Strader, J., Brodie, J. P., \& Gallagher, J. S. 2008b, MNRAS, 385, 361

Spolaor, M., Forbes, D. A., Hau, G. K. T., Proctor, R. N., \& Brough, S. 2008a, MNRAS, 385, 667 (S+08)

Spolaor, M., Forbes, D. A., Proctor, R. N., Hau, G. K. T., \& Brough, S. 2008b, MNRAS, 385, 675

Strader, J., Brodie, J. P., Spitler, L., \& Beasley, M. A. 2006, AJ, 132, 2333

Sun, M., Voit, G. M., Donahue, M., Jones, C., Forman, W., \& Vikhlinin, A. 2009, ApJ, 693, 1142

Thomas, P. A., Drinkwater, M. J., \& Evstigneeva, E. 2008, MNRAS, 389, 102

Tonry, J. L. 1983, ApJ, 266, 58

Tortora, C., Napolitano, N. R., Romanowsky, A. J., Capaccioli, M., \& Covone, G. 2009, MNRAS, in press

Tran, K.-V. H., Moustakas, J., Gonzalez, A. H., Bai, L., Zaritsky, D., \& Kautsch, S. J. 2008, ApJ, 683, L17

Trentham, N., Tully, R. B., \& Mahdavi, A. 2006, MNRAS, 369, 1375

Trujillo, I., Erwin, P., Asensio Ramos, A., \& Graham, A. W. 2004, AJ, 127, 1917

Tully, R. B. 2005, ApJ, 618, 214

van Albada, T. S. 1982, MNRAS, 201, 939

van den Bosch, F. C., Lewis, G. F., Lake, G., \& Stadel, J. 1999, ApJ, 515, 50

van den Bosch, R. C. E., van de Ven, G., Verolme, E. K., Cappellari, M., \& de Zeeuw, P. T. 2008, MNRAS, 385, 647

Vesperini, E., Zepf, S. E., Kundu, A., \& Ashman, K. M. 2003, ApJ, 593, 760

Vikhlinin, A., Kravtsov, A., Forman, W., Jones, C., Markevitch, M., Murray, S S., \& Van Speybroeck, L. 2006, ApJ, 640, 691

von Benda Beckmann, A. M., D'Onghia, E., Gottlöber, S., Hoeft, M., Khalatyan, A., Klypin, A., \& Müller, V. 2008, MNRAS, 386, 2345

von der Linden, A., Best, P. N., Kauffmann, G., \& White, S. D. M. 2007, MNRAS, 379, 867 
von der Linden, A., Erben, T., Schneider, P., \& Castander, F. J. 2006, A\&A, 454, 37

Waters, C. Z., Zepf, S. E., Lauer, T. R., \& Baltz, E. A. 2009, ApJ, 693, 463

Wechsler, R. H., Bullock, J. S., Primack, J. R., Kravtsov, A. V., \& Dekel, A. 2002, ApJ, 568, 52

Wehner, E. M. H., \& Harris, W. E. 2007, ApJ, 668, L35

Wehner, E. M. H., Harris, W. E., Whitmore, B. C., Rothberg, B., \& Woodley, K. A. 2008, ApJ, 681, 1233

Weil, M. L., \& Hernquist, L. 1996, ApJ, 460, 101

Weinmann, S. M., van den Bosch, F. C., Yang, X., \& Mo, H. J. 2006, MNRAS, 366,2

Weisskopf, M. C., Brinkman, B., Canizares, C., Garmire, G., Murray, S., \& Van Speybroeck, L. P. 2002, PASP, 114, 1
Whitmore, B. C., \& Schweizer, F. 1995, AJ, 109, 960

Willmer, C. N. A., Focardi, P., da Costa, L. N., \& Pellegrini, P. S. 1989, AJ, 98, 1531

Wilman, D. J., et al. 2008, ApJ, 680, 1009

Wojtak, R., Łokas, E. L., Gottlöber, S., \& Mamon, G. A. 2005, MNRAS, 361, L1

Woodley, K. A., Harris, W. E., Beasley, M. A., Peng, E. W., Bridges, T. J., Forbes, D. A., \& Harris, G. L. H. 2007, AJ, 134, 494

Wu, X., \& Tremaine, S. 2006, ApJ, 643, 210

Yang, X., Mo, H. J., Jing, Y. P., \& van den Bosch, F. C. 2005, MNRAS, 358, 217

Zabludoff, A. I., \& Mulchaey, J. S. 1998, ApJ, 496, 39

Zhang, Z., Xu, H., Wang, Y., An, T., Xu, Y., \& Wu, X.-P. 2007, ApJ, 656, 805 $(\mathrm{Z}+07)$ 